\title{
Solar Wind and Internally Driven Dynamics: Influences on Magnetodiscs and Auroral Responses
}

\author{
P.A. Delamere · F. Bagenal • C. Paranicas • A. Masters • \\ A. Radioti · B. Bonfond · L. Ray $\cdot$ X. Jia $\cdot$ J. Nichols • \\ C. Arridge
}

Received: 10 February 2014 / Accepted: 6 July 2014

(C) Springer Science+Business Media Dordrecht 2014

\begin{abstract}
The dynamics of the giant planet magnetodiscs are strongly influenced by planetary rotation. Yet the solar wind must ultimately remove plasma from these rapidly rotating magnetodiscs at the same rate that plasma is transported radially outward from the source regions: the Io and Enceladus plasma tori. It is not clear how the solar wind influences magnetospheric dynamics when the dynamics are dominated by rotation. However, auroral observations provide important clues. We review magnetodisc sources and radial transport and the solar wind interaction with the giant magnetospheres of Jupiter and Saturn in an attempt to connect auroral features with specific drivers. We provide a discussion of auroral signatures that are related to the solar wind interaction and summarize with a discussion of global magnetospheric dynamics as illustrated by global MHD simulations. Many questions remain and it is the intent of this review to highlight several of the most compelling questions for future research.
\end{abstract}

Keywords Neutral clouds · Plasma torus · Momentum loading · Dynamics · Aurora · Magnetodiscs $\cdot$ Reconnection $\cdot$ Solar wind

\section{Introduction}

The dynamics of giant planet magnetospheres are largely dominated by planetary rotation. The centrifugal confinement of plasma originating from Io and Enceladus, located deep in the inner magnetosphere, is fundamental to the formation of these structures. The equatorially-confined plasma carries azimuthal currents that distort the magnetic field into a thin disc-like structure called the magnetodisc (see discussion by M. Kivelson, this issue). The outward plasma transport mechanism in these rapidly-rotating magnetospheres is

P.A. Delamere $(\bigotimes) \cdot$ F. Bagenal · C. Paranicas · A. Masters · A. Radioti · B. Bonfond · L. Ray · X. Jia J. Nichols · C. Arridge

Geophysical Institute \& Physics Department, University of Alaska Fairbanks, 903 Koyukuk Drive, PO Box 757320, Fairbanks, AK 99775, USA

e-mail: Peter.Delamere@gi.alaska.edu 
thought to be the centrifugally-driven flux tube interchange instability (akin to the gravitational Rayleigh-Taylor instability). Eventually, the plasma is lost to the magnetotail, modulated by the variable upstream solar wind environment (Arridge et al. 2011; Jackman and Arridge 2011). Reconnection likely plays a key role in generating disconnected plasma blobs (plasmoids) that can be carried away by the solar wind. The Vasyliunas cycle is based on centrifugal stretching of the magnetodisc in the tail region, allowing the current sheet to thin and reconnection to operate (Vasyliunas 1983). Dayside reconnection can drive a Dungey cycle of reconnection that on long time scales requires tail reconnection to conserve flux (Dungey 1961). This solar wind-driven reconnection could be important for magnetodisc dynamics. Finally, the tangential stresses generated at the magnetopause boundary by the solar wind's viscous interaction can also influence dynamics (Axford and Hines 1961; Delamere and Bagenal 2010). All of these processes lead to mass loss from the magnetodisc.

Mass transport is a two-way process in planetary magnetospheres and cannot be considered independent of magnetic flux or internal energy transport. While net mass flux can point into or out of the magnetosphere, magnetic flux must be balanced on long time scales. At Jupiter and Saturn, roughly $500 \mathrm{~kg} / \mathrm{s}$ and $50 \mathrm{~kg} / \mathrm{s}$ are lost to the solar wind, respectively (Delamere and Bagenal 2013). During radial transport, plasma is heating non-adiabatically, requiring an input energy of 3-16 TW at Jupiter and 75-630 GW at Saturn (Bagenal and Delamere 2011). The detailed physical transport mechanisms and associated heating are poorly understood. However, ultimately the transport physics must take into consideration the interaction of the giant magnetodiscs with the solar wind.

The purpose of this article is to review the internally-driven dynamics and the solar wind interaction. We review: mass and energy transport in planetary magnetodiscs (Sect. 2), magnetopause boundary processes that facilitate the solar wind interaction (Sect. 3), and observational clues found in the auroral emissions (Sect. 4). Finally, we conclude with a discussion of what global simulations reveal about the solar wind interaction (Sect. 5) and how magnetodisc plasma is lost to the solar wind.

\section{Mass and Energy Flow in Planetary Magnetodiscs}

\subsection{Sources}

Quantifying the transport of mass and energy through planetary magnetodiscs is crucial for untangling internal and external (i.e. solar wind) drivers of magnetospheric dynamics. For example, the radial mass transport rate, $\dot{M}$, together with ionospheric conductivity characterizes the magnetosphere-ionosphere coupling that can generate internally-driven aurora (e.g. Hill 1979; Nichols and Cowley 2004; Ray et al. 2010). Non-adiabatic heating of the plasma during radial transport affects the plasma- $\beta$ at the magnetopause boundary that, in part, determines the nature of the solar wind interaction (e.g. Masters et al. 2012a). The equilibrium scale (i.e. subsolar distance of the magnetopause boundary) of the magnetosphere is dependent on the momentum transfer rate from the solar wind, requiring that plasma mass loss to the solar wind balances the internal plasma source rate (Delamere and Bagenal 2013). In this section we discuss the flow of mass and energy in the context of magnetosphere-ionosphere coupling and the superthermal plasma populations of planetary magnetodiscs.

Located deep in the inner magnetospheres of Jupiter and Saturn, Io and Enceladus are the respective sources of neutral gas that supply plasma to these planetary magnetodiscs. The fate of these neutral gases is very different. At Jupiter nearly all of the iogenic neutral gas is ionized and either removed from the system as fast escaping neutrals as a result of 
charge exchange or transported radially outward as plasma. Of the initial $\sim$ tonne per second $(1000 \mathrm{~kg} / \mathrm{s})$ of neutral gas generated by Io, only $500 \mathrm{~kg} / \mathrm{s}$ survives as plasma for the magnetodisc. At Saturn, the low thermal electron temperatures near Enceladus result in a neutral dominated gas in Saturn's inner magnetospheres. The expansion and redistribution of this neutral torus eventually supplies Saturn's magnetodisc at larger radial distances, where the electrons are hot enough to ionize. Ultimately, only $\sim 50 \mathrm{~kg} / \mathrm{s}$ of the initial $\sim 200 \mathrm{~kg} / \mathrm{s}$ of water vapor spewing from the Encledus geysers is transport radially as plasma through the magnetodisc. A complete discussion of the satellite neutral clouds and evolution of the plasma tori is given by Achilleos et al. (2014, this issue).

\subsection{Plasma Transport and Magnetosphere-Ionosphere Coupling}

At both Jupiter and Saturn, radial transport of plasma is driven primarily by centrifugal forces. In this process, which is similar to the gravitationally driven Rayleigh-Taylor instability, rapidly rotating cold, dense plasma experiences an outward centrifugal force. As the flux tubes containing cold, dense plasma move radially outward, flux tubes with hot, tenuous plasma are transported towards the planet to conserve magnetic flux, while reducing the centrifugal potential energy of the system (see review by Thomas et al. 2004). Radial transport out of the Io torus occurs very slowly, with plasma residing inside of $10 R_{J}$ for 23-50 days (Delamere and Bagenal 2003).

As plasma is radially transported outwards, conservation of angular momentum dictates that it slows in its angular motion. However, the planetary magnetic field is frozen-in to the magnetospheric plasma. Therefore, the slowing magnetospheric plasma exerts a stress on the magnetic field, bending it backwards. Simultaneously, field-aligned currents develop, supporting the magnetic field geometry and transferring angular momentum from the planetary atmosphere to the magnetospheric plasma. These currents flow radially through the magnetospheric equator, and the ensuing $\mathbf{J} \times \mathbf{B}$ forces accelerates the magnetospheric plasma towards corotation. The current circuit closes in the planetary ionosphere, with the latitudinal current directed equatorwards, thus imposing a $\mathbf{J} \times \mathbf{B}$ force in the ionosphere opposite to that in the magnetosphere. At Jupiter, the main auroral emission, observed in both IR and UV wavelengths, is a signature of this current circuit, as the upward field-aligned currents that feed the magnetospheric plasma are associated with planetward moving electrons that excite the planetary atmosphere. Low latitude aurora have also been identified on Saturn in the infrared and ultraviolet domain, which could correspond to the main emissions on Jupiter (Stallard et al. 2008; Grodent et al. 2010). While omnipresent, Jupiter's main auroral is more dynamic than originally thought; However, the constant production-and subsequent transport — of Iogenic plasma lends itself to an exploration of the steady state system, which can then be expanded to understand how the solar wind influences the main auroral emission.

Hill (1979) first explored the above scenario, predicting the radial profile of the magnetospheric plasma's angular velocity through balancing the ionospheric and magnetosphere torques. His analytic description assumed a dipole planetary magnetic field and constant ionospheric Pedersen conductance, $\Sigma_{P}$. He found that the departure from corotation of the magnetospheric plasma angular velocity could be characterized by a value,

$$
L=\left(\frac{\pi \Sigma_{P} R_{p}^{2} B_{P}^{2}}{\dot{M}}\right)^{1 / 4}
$$

where $R_{P}$ is the planetary radius, $B_{P}$ is the magnetic field strength at the ionosphere, and $\dot{M}$ is the radial mass transport rate in $\mathrm{kg} / \mathrm{s}$. For radial distance larger the $L$, the lag from corotation of the plasma would be significant. It is clear to see from Eq. (1) that the location of 
the departure from corotation depends on the ratio of the ionospheric Pedersen conductance to the radial mass transport rate.

Since the seminal analysis of Hill (1979), there have been many studies expanding the simplifying assumptions of the original analysis (e.g. Huang and Hill 1989; Pontius 1997; Cowley and Bunce 2001; Nichols and Cowley 2004; Smith and Aylward 2009; Ray et al. 2010; Yates et al. 2012). The giant planet systems are more complicated than a dipole magnetic field and a planetary atmosphere with constant Pedersen conductance. In steady state the main factors that influence the ability of the planetary atmosphere to communicate angular momentum to its surrounding magnetospheric plasma, and hence field-aligned current strengths and subsequent auroral emission location and brightness, are (1) the rotation rate of the planetary thermosphere; (2) the modification of the ionospheric Pedersen conductance with auroral electron precipitation; (3) the magnitude of the north-south component of the planetary magnetic field; and (4) the latitudinal distribution of current carriers along the magnetic field and possible accompanying potential drops.

Ultimately, in the case of Jupiter, the angular momentum that is transferred out to the magnetospheric plasma comes from the planet's deep interior. Angular momentum is transported from the deep interior to the thermosphere via advection and eddy diffusion (Smith and Aylward 2008; Huang and Hill 1989). Thermospheric flows are then coupled to the ionosphere through ion-neutral collisions. If the neutral thermosphere is significantly subcorotating, then that will affect the maximum planetary angular velocity that is communicated to the magnetosphere. The Pedersen conductance, in part, controls the magnitude of the ionospheric currents, and therefore the field-aligned currents that transport angular momentum outwards to the magnetospheric plasma. Electron precipitation into the planetary atmosphere will enhance the Pedersen conductance, acting as a positive feedback mechanism, which encourages the transfer of angular momentum (Nichols and Cowley 2004; Ray et al. 2010). In both the jovian and saturnian magnetospheres, the planetary magnetic field is distended by a current sheet that results in a decreased field strength from that of a dipole. The departure of the field from a dipole configuration depends on the plasma pressure, and will therefore change with local plasma parameters (Caudal 1986; Achilleos et al. 2010b, 2010a; Nichols 2011). Diminishing magnitude of the north-south component of the planetary magnetic field will hinder the delivery of angular momentum to the magnetospheric plasma, affecting the $\mathbf{J} \times \mathbf{B}$ force on the plasma (Pontius 1997).

Finally, both Jupiter and Saturn are rapid rotators, therefore their magnetospheric plasma experiences strong centrifugal forces. Cold, heavy ions will be confined to the equatorial plane whilst lighter ions, such as protons, and electrons will have more mobility along the magnetic field. The ensuing charge separation results in an ambipolar electric field that acts to restrict electrons in their planetward motion and pull positively charge particles up off the equatorial plane. Ionospheric plasma is restricted to high latitudes because of gravitational forces. Therefore, at high latitudes, there is a minimum in the plasma density along the magnetic flux tube (Su et al. 2003; Ray et al. 2009). Should the magnetospheric plasma's demand for angular momentum, and hence current, from the planet exceed the thermal electron current density, field-aligned potentials will develop at the location of the plasma density minimum. Changes in the magnitude of the field-aligned potentials with latitude, or equivalently equatorial radius, will modify the electric field mapping between the ionosphere and the magnetosphere, allowing for rotational decoupling between the two regions. Another effect of the field-aligned potentials is to spread the transfer of angular momentum over a broader region in the magnetospheric equator (Ray et al. 2010). Note that this process does not necessarily broaden the width of the auroral emission at the planet because of the distension of the planetary magnetic field due to the current sheet. Jupiter's sulfur dioxide-based 
chemistry, and hence lower abundance of protons relative to the Saturn's water group-based system, means that this effect is stronger in the jovian system.

At higher energies (i.e. superthermal particles) the nature of the sources of particles and their transport can be quite different from the picture we just put forward. For instance, energetic protons above a certain energy are believed to be created via the Cosmic ray albedo neutron decay (Crand) mechanism (e.g., Cooper and Simpson 1980). These particles are the decay products of neutrons created when cosmic rays have access to Saturn and its rings. Recently, Roussos et al. (2011) linked energetic proton fluxes to the phase of the solar cycle to provide more evidence for the Crand mechanism.

At higher energies, there is evidence of both the local interchange, described above for thermal plasma, and the injection of global distributions of hot ions. Mitchell (2015) described the latter type as signatures of current sheet collapse. These processes could in fact be larger scale manifestations of interchange. But at least from the point of view of in situ data, there are qualitative differences between larger scale injections, that can be imaged in ENAs, and flux tube interchange, that occurs closer to the planet.

It is also not well understood how flux tube interchange and/or current sheet collapse relate to transport on smaller spatial scale, e.g., micro-diffusion. Microdiffusion is believed to be important, for instance, in explaining the rate of fill in of satellite micro signatures. These depletions created by satellite absorptions fill in even when injections are not present. It is important to keep in mind that there are multiple modes of transport in these systems.

\subsection{Suprathermal Particles in Planetary Magnetodiscs}

The solar wind flows approximately radially outward from the Sun. The ion plasma contains light and heavy ions in various charge states, including both pick-up Hydrogen $\left(\mathrm{H}^{+}\right)$ and Helium (i.e., $\mathrm{He}^{+}$) and solar Hydrogen $\left(\mathrm{H}^{+}\right)$and $\mathrm{He}^{++}$(alpha particles). The pickup ions are derived from interstellar neutrals. Helium ions found in planetary magnetospheres provide a good tracer of the access of solar wind. For instance, Haggerty et al. (2009) have examined the relative proportion of the main energetic ions as a function of distance down Jupiter's magnetotail. They show that closer to the planet, the charged particles become more dominated by Iogenic composition, but also reveal the deep penetration of solar wind Helium.

The velocity distribution of the quiescent solar wind electrons can be modeled using a Maxwellian for the cold plasma core, a kappa distribution for the halo (Vasyliunas 1968), and a power law for the energetic tail (see Fig. 1). The velocity distribution of solar wind $\mathrm{H}^{+}$ measured in the solar wind at 5.2 AU (appropriate for Jupiter) also includes an interstellar pickup proton population (Fig. 2).

The kappa distribution is used throughout the planetary magnetospheres and the heliosphere to describe suprathermal particles (e.g., Dialynas et al. 2009; Decker et al. 2005). It is like a Maxwellian, but at small kappa parameters, it deviates in important ways (see Fig. 3). The suprathermals can often play a leading role in the total particle pressure in planetary magnetospheres (Sergis et al. 2010), and not surprisingly because it is a pressure-driven current, the planetary ring current (Krimigis et al. 2007). Particles that generate auroral signatures are typically in the suprathermal energy range.

Kappa distributions are observed regularly in magnetospheric contexts but the source of these distributions is not well understood. In their very useful paper, Pierrard and Lazar (2010) outline some of the theory behind the distribution and its applications in the solar system. They point out, for instance, that, "an isotropic kappa distribution (instead of a Maxwellian) in a planetary (context) leads to a number density $n$ decreasing as a power law 
Fig. 1 Electron distribution of the quiet time solar wind from Wang et al. (2012)
Fig. 2 Proton velocity distribution (plot symbols) measured in the quiet solar wind by Ulysses at an average helioradius of 5.2 AU. In addition to the core, halo and power-law tail components, the proton distribution includes pickup protons whose distribution is flat from low velocities up to about twice the solar wind speed (the ordinate has dimensions $\mathrm{s}^{3} \mathrm{~km}^{-6}$ ) (Mason and Gloeckler 2012)
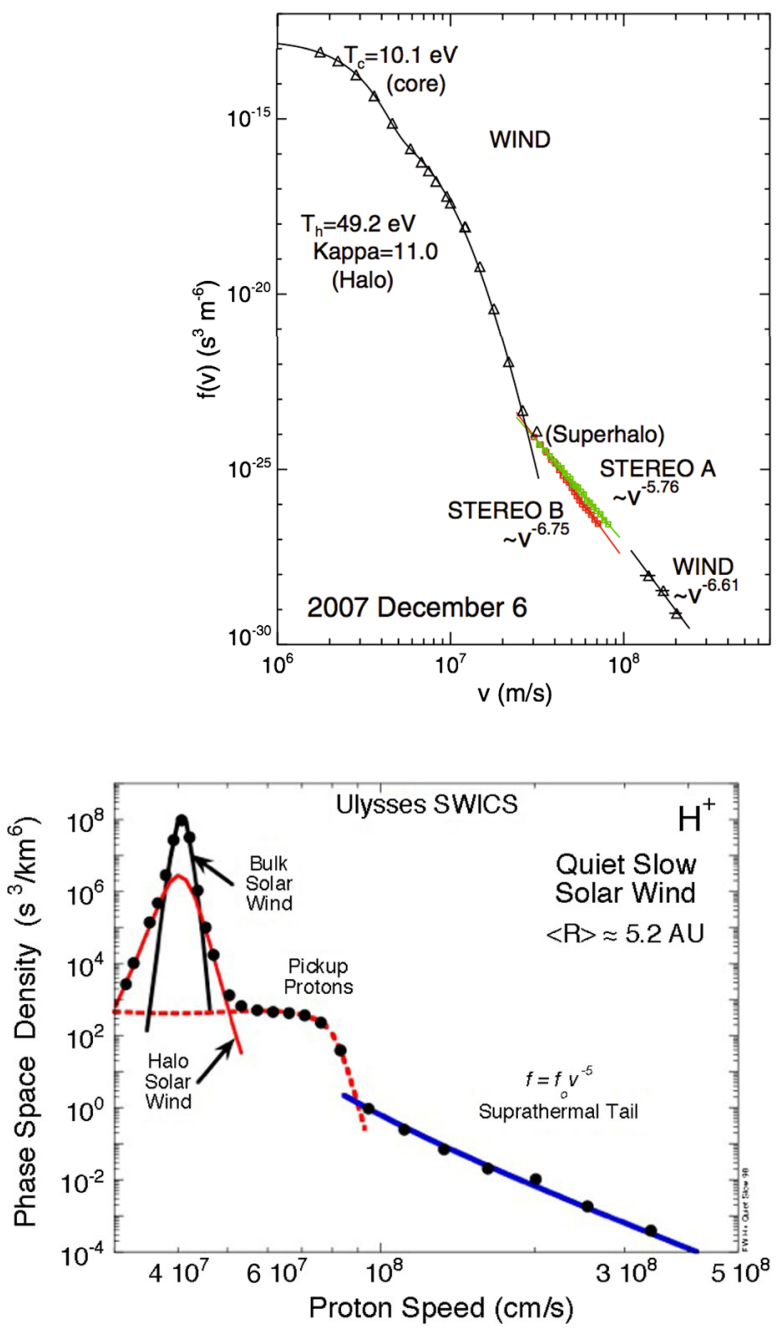

(instead of exponentially) with the radial distance $r$ and a temperature $T$ increasing with radial distance (instead of being constant)..."

In addition to how the distribution may evolve as a function of radial distance from the planet, it is also worth mentioning the important role of neutrals on suprathermal populations. Neutrals from Enceladus populate a wide region of the inner and middle magnetosphere of Saturn. These neutrals can cause suprathermal singly-charged ions to be lost from the magnetosphere via charge exchange. In this process, the more energetic ion leaves the system as an energetic neutral atom, leaving behind a cold ion. In fact, data reveal that in Saturn's inner magnetosphere, very low fluxes of trapped ions are present, except right at the corotation speed (Young et al. 2005, their Fig. 1). Corotation speed ions also undergo charge-exchange with ambient neutrals but this only replaces one thermal ion with another. Paranicas et al. (2008) also found that energetic ions can be largely absent in Saturn's inner magnetosphere due to charge exchange. They show that often the energetic ions that are detected are due to fresh injections. 
Fig. 3 From the work of Pierrard and Lazar (2010) showing examples of the kappa distribution for different kappa parameters. This figure illustrates that as kappa goes to infinity, the distribution is a Maxwellian

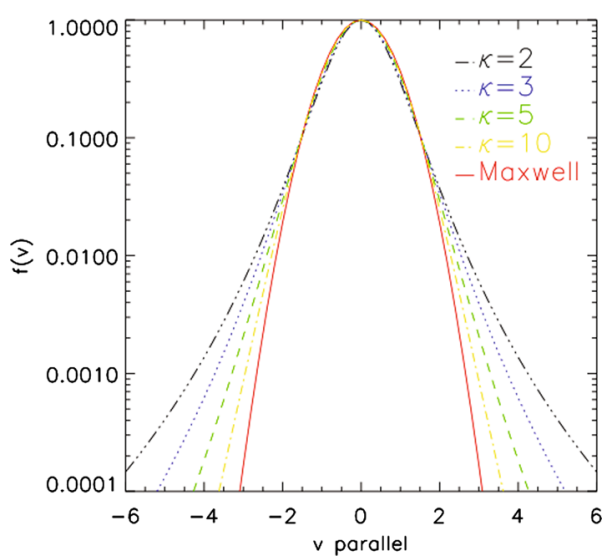

The situation for suprathermal electrons at Saturn is somewhat different. These particles can be energized by heating from the cold plasma range (for instance through Coulomb collisions with the corotating ions) and by invariant conservation as part of the inward motion that takes place during injections/interchange. Suprathermal electrons can lose energy in interactions with the neutral gas. They scatter off of all the charges in the gas/dust medium and lose energy by, for instance, providing excitations to the atoms and molecules in the medium. They are also efficient at ionizing and lose energy this way.

To summarize, the role of suprathermals in the inner magnetospheres of Jupiter and Saturn is probably vastly different. At Jupiter, the distribution function of charged particles is probably very robust between the cold plasma and the energetic particle range outward of the confined neutral gas distributions. However, at Saturn, there are important losses of ions in the suprathermal energy range and above to charge exchange with the expanded neutral gas distribution (Cassidy and Johnson 2010; Fleshman et al. 2012). Furthermore, even suprathermal and higher energy electrons at Saturn can be cooled by the presence of the neutral gas. Sergis et al. (2010), for instance, showed that the corotating cold plasma is very critical in the inner magnetosphere of Saturn and more energetic particles start to play a role in the pressure at larger distances.

\section{Magnetopause Boundary Processes}

The question of exactly how the solar wind interacts with the giant planet magnetospheres has been a major subject of debate. Although we have a significant amount still to learn about this topic, recent studies have caused our understanding to evolve.

At the magnetopause boundary of a planetary magnetosphere there is a direct interaction between the shock-processed solar wind plasma and the planetary magnetized plasma environment. Understanding the solar wind-magnetosphere interaction at any planet must be based on a clear understanding of the physical processes operating at the magnetopause. These processes lead to mass, momentum, and energy transport into the magnetosphere.

In this section we discuss the processes operating at the magnetopause boundary of both Jupiter's and Saturn's magnetosphere. As we will see, current understanding suggests that the nature of these processes varies between planets, making the assumption of an Earth-like solar wind interaction very likely invalid. 


\subsection{Magnetic Reconnection}

One of the major magnetopause processes responsible for energy transport is magnetic reconnection (Dungey 1961; Vasyliunas 1975; Russell 1975) (see the review by Paschmann 2008 and references therein). This is a fundamental process where plasma and coupled magnetic field lines flow toward a reconnection 'x-line', where they become decoupled and are essentially 'cut' and 'reconnected', before flowing away from the $\mathrm{x}$-line and recoupling with the plasma. This process changes the topology of the magnetic field and releases magnetic energy. In the case of Earth's magnetosphere, reconnection at the magnetopause is the principal driver of energy flow through the magnetosphere.

The physics of the reconnection process remains the subject of much research. It is wellestablished that the reconnection rate decreases as the ratio of plasma to magnetic pressure (the plasma $\beta$ ) adjacent to the current sheet increases (e.g. Sonnerup and Ledley 1974; Anderson et al. 1997); however, recent work suggests that certain plasma conditions can suppress the onset of reconnection itself. An increasing difference in plasma $\beta$ across a current sheet has been shown to suppress reconnection onset for an increasing range of magnetic shears across the boundary (Phan et al. 2010, 2013). This is due to the potential for particle drifts within the current sheet to prevent the flow pattern required for reconnection from being established (Swisdak et al. 2003, 2010). This effect is hereafter referred to as diamagnetic suppression. In addition, it has been suggested that a super-Alfvénic flow shear in the direction of the reconnecting field can also suppress reconnection, similarly related to disruption of reconnection-imposed flows (e.g. Cassak and Otto 2011). This effect is hereafter referred to as flow shear suppression.

We know the Mach numbers of planetary bow shocks increase with distance from the Sun (e.g. Russell et al. 1982), which produce different solar wind plasma conditions adjacent to each planetary magnetopause. We also know that there is non-negligible plasma pressure in the outer magnetospheres of Jupiter and Saturn, combined with significant flows in the sense of planetary rotation. When considering the nature of magnetic reconnection at both Jupiter's and Saturn's magnetopause, an assessment of the effect of these differing conditions is essential.

Spacecraft observations at Jupiter's magnetopause have revealed evidence for the occurrence of reconnection (Walker and Russell 1985; Huddleston et al. 1997). Identification of encounters with the reconnection-related phenomenon of Flux Transfer Events (FTEs) (Russell and Elphic 1978; Walker and Russell 1985) is a particularly clear indication that the process does operate. However, spacecraft observations at Jupiter's magnetopause remain limited, meaning that we cannot yet build a statistical picture of where, and under what conditions, reconnection occurs.

McComas and Bagenal (2007) argued that the long time scale associated with an open field line being dragged to the magnetotail by the solar wind compared to the time scale of Interplanetary Magnetic Field (IMF) sector changes means that such open field lines can be closed through further magnetopause reconnection. The proposed ability of this scenario to keep the amount of open flux in the system small was challenged by Cowley et al. (2008), primarily on the basis of a comparison of expected rates of magnetic flux opening and closure due to magnetopause reconnection at different locations. This concept remains the subject of debate (McComas and Bagenal 2008).

Most recently, Desroche et al. (2012) used idealized models of conditions at Jupiter's magnetopause to draw conclusions about the solar wind-magnetosphere interaction. Included in their investigation was an assessment of how favorable conditions at Jupiter's magnetopause are for reconnection onset (see Fig. 4). They found that the large flow shears 
Fig. 4 Assessment of how favorable conditions at Jupiter's magnetopause are for magnetic reconnection onset. In all panels the magnetopause surface is viewed from along the solar wind flow direction. The panel surrounded by a blue rectangle shows an assessment of flow shear suppression, and the panels surrounded by a red rectangle show assessments of diamagnetic suppression (for different values of the plasma $\beta$ in the magnetosphere, $\left.\beta_{M S P}\right)$. Regions of each surface shaded in red are regions where reconnection onset is possible. Adapted from Desroche et al. (2012)
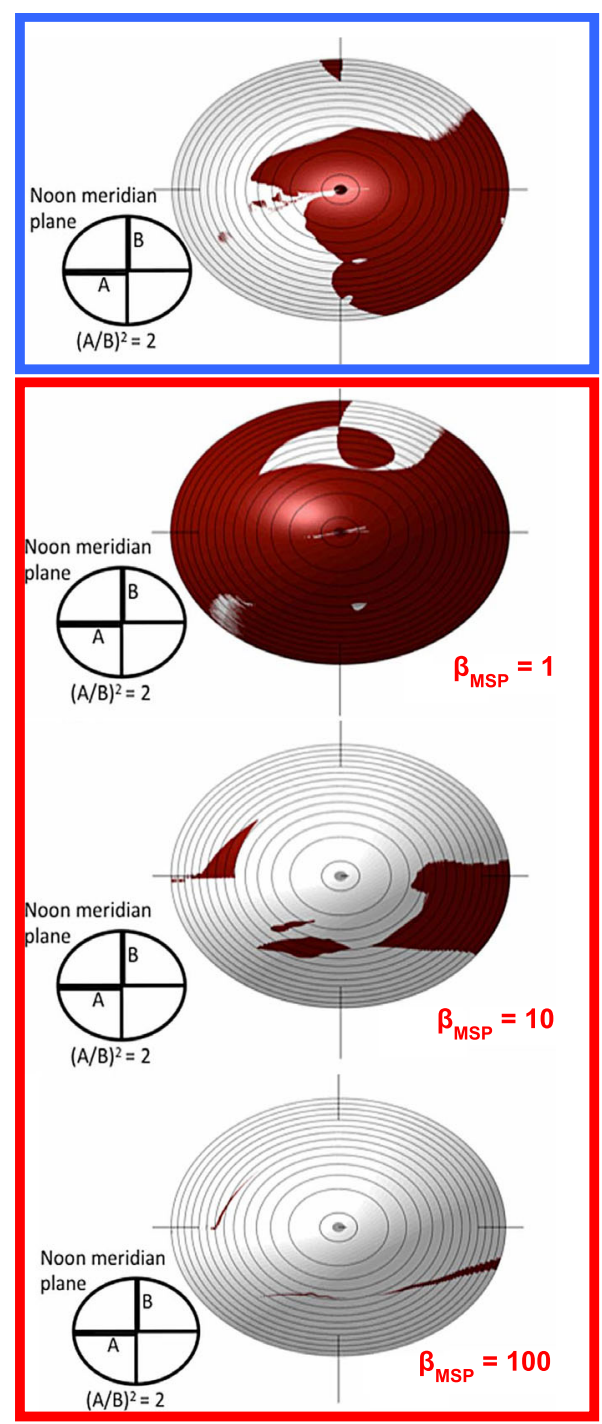

across the dawn flank magnetopause generally prohibit reconnection due to flow shear suppression. Furthermore, by considering different values of the (poorly constrained) plasma $\beta$ in Jupiter's near-magnetopause magnetosphere they showed that diamagnetic suppression may also be severe.

In the case of Saturn's magnetosphere, in situ evidence for magnetopause reconnection has also been reported (Huddleston et al. 1997; McAndrews et al. 2008; Lai et al. 2012; Fuselier et al. 2014), and it has been suggested that some dayside auroral features are caused by bursts of magnetopause reconnection (Radioti et al. 2011a; Badman et al. 2012a, 2013). However, unlike Jupiter, no examples of FTEs have been identified to date (Lai et al. 2012), and neither Saturn's auroral power nor the thickness on the magnetospheric boundary layer adjacent to the magnetopause show a clear response to the orientation of the IMF (unlike 
the case of Earth's magnetopause reconnection-driven magnetosphere) (Crary et al. 2005; Clarke et al. 2009; Masters et al. 2011).

Indirect evidence for dayside magnetopause reconnection at Saturn has been provided by in situ observations by Cassini at high latitudes in the magnetosphere. The in situ data shows the presence of magnetosheath plasma at the expected location of the cusp deep within the magnetosphere and with dispersions characteristic of magnetopause reconnection. Jasinski et al. (2014) have used ion energy-pitch angle dispersions to argue for a magnetopause reconnection interpretation using data from the northern hemisphere. Furthermore, they observed stepped time-energy ion dispersions that argue for pulsed reconnection, as suggested by the rapid variations in clock angle inside CIRs (Jackman et al. 2005).

The southern polar cusp was studied using in situ data from Cassini in January and February 2007 (Arridge et al. 2014). In these events energy-pitch angle dispersions were also suggested for magnetopause reconnection. These case studies demonstrated injections in the cusp under a range of solar wind dynamic pressure conditions thus showing that the solar wind interaction has an important component under more rarefield solar wind conditions. The in situ data also contains evidence for a boundary layer between the open field lines in the cusp and the field-aligned currents driving the main auroral emission thus suggesting that the main auroral emission and the OCB are not exactly co-located. Evidence was also presented that the cusp oscillated in position in phase with Saturn's global magnetospheric periodicities.

Note that although spacecraft observations in the vicinity of Saturn's magnetopause are currently more extensive than those made at Jupiter's magnetopause, the Saturn data sets also suffer from limited instrument fields-of-view. This almost always prohibits the assessment of whether or not the clearest evidence for magnetopause reconnection in the form of reconnection outflows (jets) were present at a spacecraft magnetopause encounter. As a result, a comprehensive statistical picture of where and why magnetopause reconnection occurs is also not available for Saturn's magnetopause.

However, Masters et al. (2012a) assessed the diamagnetic suppression condition at Saturn's magnetopause using spacecraft observations of local plasma $\beta$ conditions at the boundary. They found that diamagnetic suppression is likely to be severe, primarily due to high- $\beta$ conditions in the solar wind adjacent to Saturn's magnetopause (see Fig. 5). This high- $\beta$ environment is consistent with the high-Mach number of Saturn's bow shock. It is thus expected that reconnection at Saturn's magnetopause is generally limited to regions of the magnetopause surface where the fields on either side of the boundary become almost antiparallel (note that this is presently consistent with observed reconnection signatures, e.g. Fuselier et al. 2014). More recently, Masters et al. (2014) reported the results of a study that was similarly based on measured near-magnetopause conditions. These authors inferred reconnection electric field strengths (assuming the satisfaction of onset conditions), and suggested that the resulting reconnection voltages applied to Saturn's magnetosphere are generally not high enough to represent a major driver of system dynamics.

As we have discussed, near-magnetopause plasma conditions at both Jupiter and Saturn differ from those at Earth's magnetopause. Faster flows in the magnetosphere can suppress reconnection, and higher plasma $\beta$ on either side of the boundary can also limit the onset of the reconnection process. Overall, current understanding suggests that the coupling between the solar wind and both Jupiter's and Saturn's magnetospheres via magnetopause reconnection is weaker than that at Earth. How Jupiter and Saturn compare with each other remains unclear. 


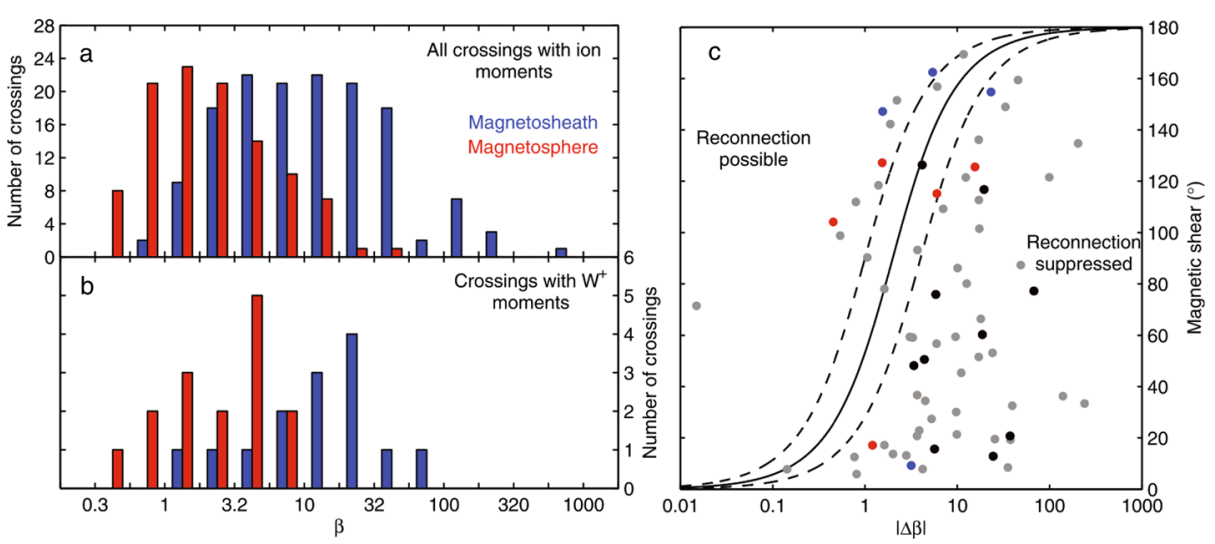

Fig. 5 Measured plasma $\beta$ conditions at Saturn's magnetopause and an assessment of the importance of diamagnetic suppression of magnetopause reconnection. Taken from Masters et al. (2012a)

\subsection{Shear-Flow Driven Instabilities}

Energy transport across the magnetopause can also be achieved through the growth of instabilities that are driven by the flow shears often present at the boundary. The most well studied of these is the Kelvin-Helmholtz (K-H) instability (e.g. Dungey 1955; Chandrasekhar 1961). This is a fundamental instability that can grow in a wide range of fluid environments. For sufficiently high flow shear across a boundary between two fluids, like a planetary magnetopause, the boundary can become K-H unstable. A seed perturbation of a K-H unstable interface will grow with time, rather than be suppressed, leading to surface waves in the linear phase of the instability, and complex boundary vortices in the subsequent nonlinear phase. There is substantial evidence for the development of K-H-driven waves and vortices on Earth's magnetopause (e.g. Hasegawa et al. 2004).

In this planetary magnetopause context, the role of K-H instability in promoting the growth of other instabilities has received much research attention, in particular magnetic reconnection (Nykyri and Otto 2001; Nakamura and Fujimoto 2005; Nakamura et al. 2006). Reconnection within rolled-up K-H vortices has been observed at Earth's magnetopause (Nykyri et al. 2006; Hasegawa et al. 2009), and conditions in these complex structures is expected to enhance plasma diffusion across the interface (e.g. Cowee et al. 2010). In case of the terrestrial magnetosphere, solar wind energy transport into the magnetosphere under northward IMF conditions is thought to occur primarily through the flanks of the magnetopause as a result of the growth of the K-H instability (e.g. Hasegawa et al. 2004).

The different plasma conditions at the magnetopause of both Jupiter and Saturn compared to at Earth's magnetopause are expected to also affect shear flow-driven instabilities like the K-H instability. Although the magnetosheath flow is similar, magnetospheric flows just inside each giant planet magnetopause are appreciable, and directed from dawn to dusk, having a strong effect on the flow shear. In addition, different plasma densities and magnetic field strengths and orientations can affect the K-H stability of these boundaries (e.g. Chandrasekhar 1961; Southwood 1968). It is reasonable to expect that the dawn flank of both Jupiter's and Saturn's magnetopause is K-H unstable, due to the larger flow shear, and thus that more K-H-related perturbations should be present in this region (Galopeau et al. 1995).

Wave activity on Jupiter's magnetopause has been identified based on multiple spacecraft crossings of the boundary (Huddleston et al. 1997); however, as for investigation of other 
Fig. 6 Assessment of the stability of Jupiter's magnetopause to the growth of the Kelvin-Helmholtz (K-H) instability. In all panels the magnetopause surface is viewed from along the solar wind flow direction, and shaded regions of each surface are regions predicted to be K-H unstable. Different panels consider different levels of magnetospheric polar flattening. Adapted from Desroche et al. (2012)

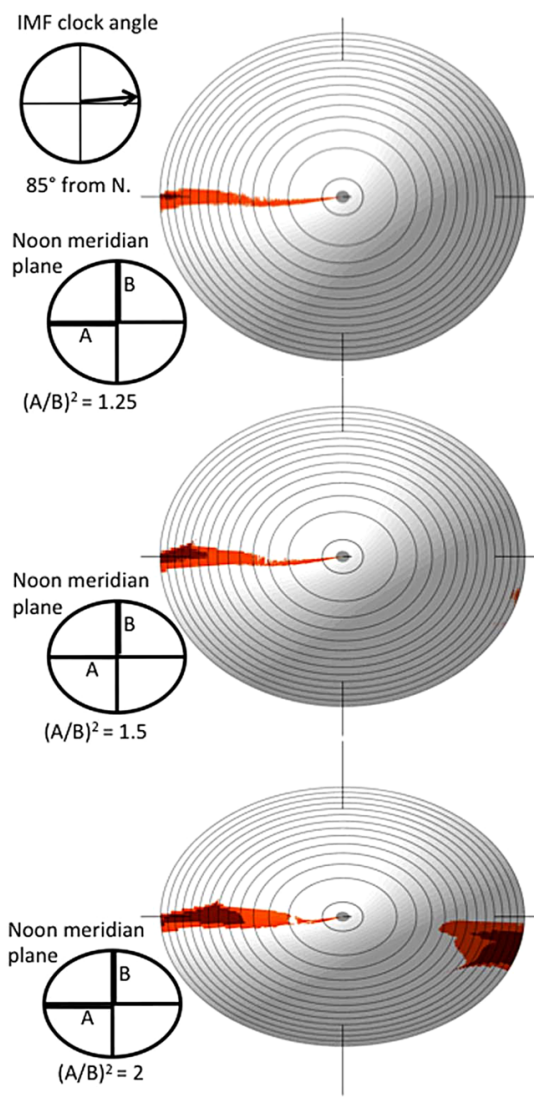

processes operating at Jupiter's magnetopause, limited spacecraft data sets prevent large statistical analyses. Note that separating wave-driving mechanisms is difficult, i.e. solar wind pressure fluctuations can cause waves as well as K-H instability growth.

In their assessment of what conditions at Jupiter's magnetopause mean for how the solar wind interacts with the magnetosphere, Desroche et al. (2012) consider the K-H stability of the boundary. They found that polar flattening of the magnetosphere (caused by centrifugal confinement of magnetospheric plasma in roughly the plane of the planetary equator) can have a significant effect on the flow in the magnetosheath, and that, as expected, the dawn flank of the magnetopause should be far more K-H unstable than the dusk flank, due to the difference in flow shears (see Fig. 6).

Delamere and Bagenal (2010) suggested that solar wind driving of Jupiter's magnetosphere is predominantly due to viscous processes, like growth of the K-H instability, operating at the magnetopause. This model is akin to that of Axford and Hines (1961). Delamere and Bagenal (2010) suggested that rather than a global cycle of reconnection where flux is opened at the dayside magnetopause and closed in the magnetotail, flux is predominantly opened and closed intermittently in small-scale structures in turbulent interaction regions on the flanks of the magnetosphere. $\mathrm{K}-\mathrm{H}$ vortices and associated reconnection is a key element of this understanding of Jupiter's magnetospheric dynamics.

Statistical studies of perturbations of Saturn's magnetopause have provided a clearer picture of K-H instability growth at this magnetospheric boundary. Case studies initially re- 
Fig. 7 Location of potential signatures of Kelvin-Helmholtz (K-H) vortices on Saturn's magnetopause. Approximately the planetary equatorial plane is shown, with the center of Saturn at the origin, and the $x$-axis pointing towards the Sun. Dashed curves indicate the range of possible locations of Saturn's magnetopause. The Cassini spacecraft trajectory is shown as a solid gray line. The locations of identified signatures are indicated, and their color indicates whether they correspond to occasions when the spacecraft was in the magnetosheath or the magnetosphere. Taken from Delamere et al. (2013)

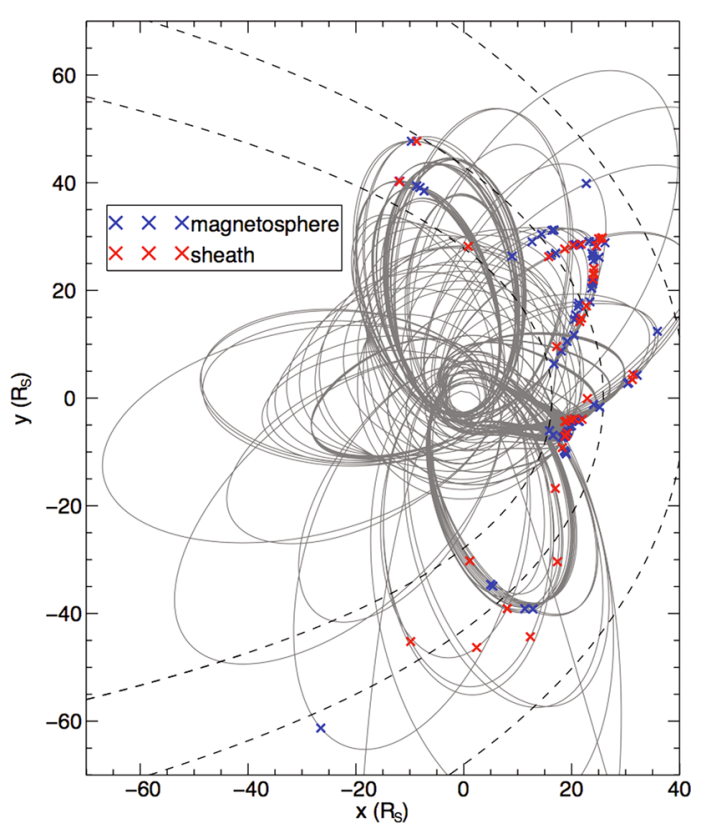

vealed evidence for waves on both the dawn and dusk sides of the magnetopause (Huddleston et al. 1997; Masters et al. 2009; Cutler et al. 2011), before a recent statistical study by Masters et al. (2012b) showed that waves are a common feature of Saturn's magnetopause dynamics. Contrary to our expectation of a dawn-dusk asymmetry, roughly equal wave occurrence was found at dawn and dusk. The tailward propagation of the identified waves, generally perpendicular to the local magnetospheric magnetic field, suggests that the K-H instability is a dominant wave driving mechanism. Most recently, Mistry et al. (2014) used simple modeling to propose that the amplitude of $\mathrm{K}-\mathrm{H}$ waves is generally comparable to the amplitude of global boundary oscillations at a period close to that of planetary rotation. A case study of a spacecraft encounter with a K-H vortex near the boundary of Saturn's magnetosphere was presented by Masters et al. (2010), and analysed in further detail by Delamere et al. (2011) and Wilson et al. (2012). Masters et al. (2010) suggested that such structures can generate spots of auroral emission, which are similar to observed auroral features (Grodent et al. 2011). A more comprehensive study of K-H vortices was recently reported by Delamere et al. (2013). These authors identified the distinctive magnetic field signatures of K-H vortices to identify many possible vortex events, which mostly occurred on the dusk flank, also contrary to expectations (see Fig. 7). Delamere et al. (2013) suggested that this is because $\mathrm{K}-\mathrm{H}$ perturbations grow in the prenoon to dusk region, before moving to dusk with the local centre of mass where they are encountered as vortices.

Numerical models have also been used to address the Saturn magnetopause K-H instability problem. Magnetohydrodynamic simulations of Saturn's magnetosphere suggest $\mathrm{K}-\mathrm{H}$ vortex formation at both dawn and dusk (Fukazawa et al. 2007a, 2007b; Walker et al. 2011), although the reason for this may differ from the reason for the observed absence of the expected local time asymmetry. In addition, hybrid simulations suggest appreciable vortex-induced diffusion and energy transport across Saturn's magnetopause (Delamere et al. 2011), which could drive magnetospheric dynamics.

These studies of shear-flow-driven instabilities at the magnetopauses of Jupiter and Saturn suggest some differences with the terrestrial case, which remain to be comprehensively 
explained. However, it is very likely that the growth of these instabilities is widespread, particularly in the case of Saturn where statistical observation based on spacecraft observations have been carried out.

\section{Auroral Signatures}

\subsection{Dynamics of Internally-Driven Aurora}

Auroral emissions at Jupiter and Saturn originate from the impact of charged particles in their hydrogen-dominated atmosphere (see review of the atmospheric response to these charged particles in Badman et al. 2014a). The aurora at Jupiter is generally described as formed of three components, the satellite footprints, the main oval and the polar emissions. A fourth component should however be added to this trio: the outer emissions, i.e. the emissions located equatorward from the main oval, but not directly related with the satellite footprints. All these components vary with time, often independently. But sometimes the combination of the observed changes proves to be a valuable tool to unveil the large scale magnetospheric processes at play. The auroral processes that dominate at Jupiter are also present at Saturn. For example, Enceladus also creates an auroral footprint on Saturn (Pryor et al. 2011). Additionally, low latitude aurora have also been identified on Saturn in the infrared and ultraviolet domain, which could correspond to the main emissions and to the outer emission on Jupiter, respectively (Stallard et al. 2008; Grodent et al. 2010).

\subsubsection{The Satellite Footprints}

At Jupiter, satellite footprints are the auroral signature of the electro-magnetic interaction between the Galilean moons Io, Europa and Ganymede on the one hand and the Jovian magnetosphere on the other hand. They appear as individual or a series of auroral spots located close to the field lines connected to moons, sometimes accompanied with arcs mapping to the moons' orbit (see review by Bonfond 2012). While the location of the Io footprint appears to remain constant with time for a given System III longitude, the Ganymede footprint may shift from one epoch to another (see Fig. 8). This shift is the consequence of changes in the magnetic field mapping and an equatorward motion corresponds to variations of either the current sheet density or thickness (Grodent et al. 2008). Ganymede and its footprint thus constitute precious landmarks to disentangle motions of auroral features owing to the magnetic field topology from those owing to motion of the source region in the magnetosphere.

Based on ultraviolet observations from the UVIS instrument on board Cassini, Pryor et al. (2011) identified the presence of the Enceladus footprint on three pseudo-images of the northern polar region. However, this footprint is particularly weak and cases of nondetection far outnumber the positive detections.

\subsubsection{Outer Emissions}

The outer emissions generally designate the auroral emissions located equator-ward from the main oval, but excluding the satellite footprints. They appear in three form: arcs, patches and diffuse emissions. It is thought that these outer emissions are caused by two different mechanisms: pitch angle diffusion and inward injections of hot plasma.

Based on simultaneous in-situ and auroral observations by Galileo and HST, Radioti et al. (2009a) attributed the faint arcs and elongated diffuse emissions to electrons being diffused in the loss cone at the pitch angle diffusion boundary. 

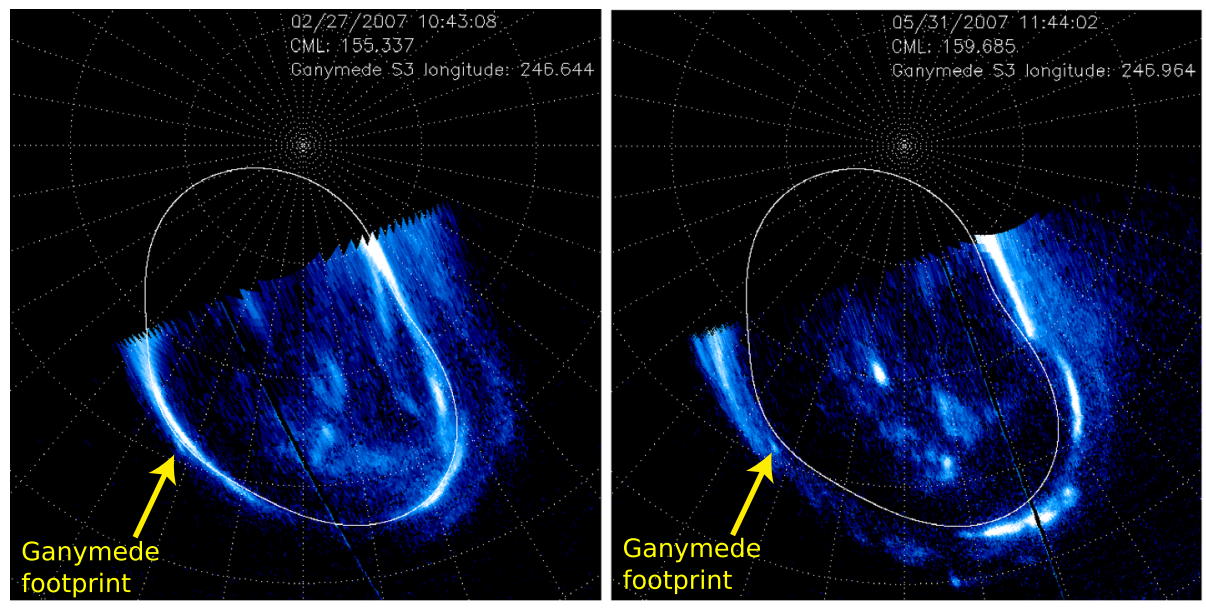

Fig. 8 Polar projection of the northern Jovian hemisphere aurora on February 27th (left panel) and on May 21st 2007 (right panel). The observing geometry was very similar, with CMLs of $155.3^{\circ}$ and $159.7^{\circ}$ respectively and a Ganymede S3 longitude of $246.6^{\circ}$ and $247.0^{\circ}$ respectively. Nevertheless, the Ganymede footprint is outside the main emission in the first image and inside it in the second case, suggesting that the source region has moved inside the Ganymede orbit $\left(15 R_{J}\right)$. Additionally, the Ganymede footprint location moved $0.5^{\circ}$ equatorwards implying an increased stretching of the magnetic field lines. The white line is the reference oval from February 2007

The patchy blobs, appearing either individually or in groups, have been attributed by Mauk et al. (2002) to injections of hot plasma coming from the outer magnetosphere and moving rapidly radially inward as a counterpart to the cold plasma moving out of the inner magnetosphere. Cassini energetic electron measurements (Radioti et al. 2009b) and numerical simulations together with simultaneous UV and ENA emissions (Radioti et al. 2013b) also indicate that injected plasma populations can create auroral emissions at Saturn. These studies suggest that, in the Kronian system, pitch angle diffusion associated with electron scattering by whistler-mode waves is the main driver of the UV auroral emissions associated with injections, while field aligned currents driven by the pressure gradient along the boundaries of the cloud might have a smaller contribution.

The morphological differences between these features is not always clear and some studies have mixed these two types of emissions under the generic terms of low-latitude or outer emissions. The brightness of the outer emissions does not seem to be correlated with the solar wind input, but appears to have considerably increased as the main oval expanded in spring 2007 (Nichols et al. 2009a; Bonfond et al. 2012). At Saturn, Grodent et al. (2010) reported the presence of a diffuse outer auroral oval, based on ultraviolet images from HST. Such an oval would map between 4 and $11 R_{S}$ and could be related to the precipitation of hot electrons diffusing in the loss cone.

\subsubsection{Main Emissions/Main Oval}

The brightest feature of the jovian aurora is a ring of emission centered on each magnetic pole, which is usually called the main oval or main emissions. Indeed, the oval usually appears broken and sometimes shows forks and parallel arcs. Moreover, the part of the main emissions corresponding to the pre-noon sector is generally 5 to 10 times weaker than the remaining of the aurora in the UV domain (Radioti et al. 2008a). While the UV and infrared 
main emissions are colocated, the brightness variations along the oval do not appear correlated (Radioti et al. 2013a). In the X-rays, the signature of the main emission differs from those of the polar emissions. The photons originating from the main oval are more energetic $(>2 \mathrm{keV})$ and caused by electron bremsstrahlung while the softer photons $(<2 \mathrm{keV})$ from the polar regions are related to the precipitation of highly stripped heavy ions (BranduardiRaymont et al. 2008). The origin of the main emissions is related to the corotation breakdown, the large scale magnetosphere-ionosphere coupling and the associated field-aligned currents (e.g. Nichols 2011; Ray et al. 2012 and reference therein).

Gustin et al. (2004) analysed far UV spectra from the STIS instrument on-board HST and studied the relationship between the brightness of the emissions and the methane absorption (through color ratios). They showed that the energy flux of the precipitating electrons was usually (but not always) correlated to the energy of these electrons, as expected from an acceleration caused by field aligned currents. They also showed that this correlation was much less clear for the polar emissions. They did not show any systematic dependence of the electron energy with local time, but the orientation of the observations was always similar (i.e. Central Meridian Longitude around $160^{\circ}$ for the north and around $80^{\circ}$ for the south).

Observed in the early FUV images of the jovian aurora (Ballester et al. 1996; Clarke et al. 1998) and then regularly afterwards, these dramatic enhancements of the dawn arc of the main emissions are still poorly understood. Gustin et al. (2006) found that while exclusively found at dawn, the brightness enhancements appeared to rotate with the magnetic field. They also showed that the brightness and the color ratio of these storms are correlated on timescales of tens of minutes. Moreover, during the large HST campaign in spring 2007, Nichols et al. (2009a) did not notice any relationship between the occurrence of dawn storms and the solar wind, contrary to other features (see below).

A correlation of the auroral emitted power with the solar wind pressure has been reported by Nichols et al. (2007), Clarke et al. (2009), Nichols et al. (2009a) (see Fig. 9), even if it is lower than at Saturn. This difference between the two planets was expected since the main emissions at Jupiter result from currents related to the corotation lag of outward drifting plasma. Actually, first order models predicted that solar wind compression regions would induce an increase in the angular velocity of the equatorial plasma and decrease the currents related to the lag from corotation, thus resulting in a dimmer aurora (e.g. Southwood and Kivelson 2001). Contrary to these expectations, Nichols et al. (2007) reported a brightening of the main emission corresponding to a period when the magnetosphere first modestly shrunk and then expanded, based on images acquired in 2000 while Cassini was upstream of Jupiter. Clarke et al. (2009) compared the brightness of the whole jovian aurora with solar wind conditions during the large 2007 HST campaign and came to a similar conclusion, i.e. a correlation of the auroral brightness with the solar wind pressure. Using the same 2007 data set, Nichols et al. (2009a) separated the aurora into distinct regions: the low latitude emissions, the main emissions, and the high latitude emissions, in order to identify the component of the aurora which responded the most to solar wind input. The outer region does not appear to be correlated with the solar wind, but enhancements of the main emissions and, to a lesser extent, of parts of the polar emissions are associated with solar wind compression regions, as in Nichols et al. (2007).

The solution of this issue may lie in the detailed timing of the response to the arrival of a compression region, as more detailed models suggest that it may result in successive phases of auroral dimming and brightening (Cowley et al. 2007). Indeed, estimates of the solar wind condition at Jupiter based on Earth-based measurements has always been challenging; the most tricky part being the prediction of the arrival time of the shocks. The delay between 


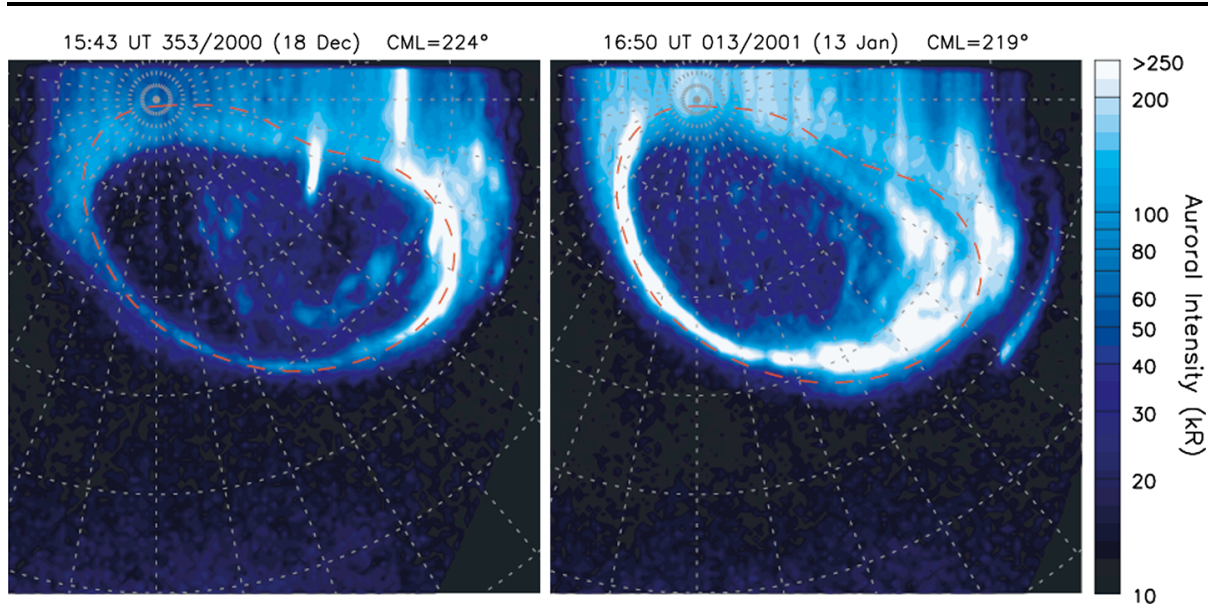

Fig. 9 HST images obtained during the Cassini Jupiter fly-by epoch and corresponding to a solar wind rarefaction region (left) and compression region (right) (adapted from Nichols et al. 2007)

the arrival of a compression region at the dayside front of the magnetopause and the auroral response is thus unclear with the current dataset.

The radial distance where the main emissions are mapping appears to vary with both local time and time. Grodent et al. (2003a) studied the location of the main oval on five sets of images from the northern hemisphere and reported a shift in its location as a function of CML. Since System III-fixed features appear to migrate poleward as the planet rotates, these images suggest that the L-shells related to the main emissions are closer at dusk then at dawn. The authors explained this difference with a different mass outflow rate as a function of the local time. Vogt et al. (2011) built a local time dependent magnetic field mapping model to map and interpret the auroral emissions located poleward of the Ganymede footprint. One of their main results was that the main emissions mapped closer to Jupiter at dawn rather than at dusk, contrary to the previous conclusion.

Additionally, Grodent et al. (2008) has shown that the location of the main emissions, even when observed in very similar configurations, could significantly (equivalent of $3^{\circ}$ of latitude) change from one observation to another. On the same pair of images, the location of the Ganymede footprint had also shifted in the same direction. Based on images from the large HST campaign from spring 2007, Bonfond et al. (2012) also studied the location of the main emissions and showed that the main oval location continuously expended from February to June. On top of this long-term trend, the location of the main emissions showed day-by-day variations. Both studies showed that the Ganymede footprint had also moved in the same direction as the main oval, indicating that the apparent motion of the main oval was at least partially due to a stretching of the magnetic field lines. At the same time, these authors noticed that the occurrence of very bright outer emissions also increased from February to June. They interpreted these events as the outcome of an increased Io torus and plasma sheet density caused by an increased volcanism on Io, supporting the conclusions of Nichols (2011). This enhanced loading of the system would lead to both an increase of the plasma sheet density, explaining the shift of the Ganymede footprint, and an increase of the plasma outflow rate, explaining the expansion of the main oval. Moreover, an increased injection occurrence rate, in order to conserve the magnetic flux in the inner magnetosphere, would explain the increased occurrence of large injection blobs. 


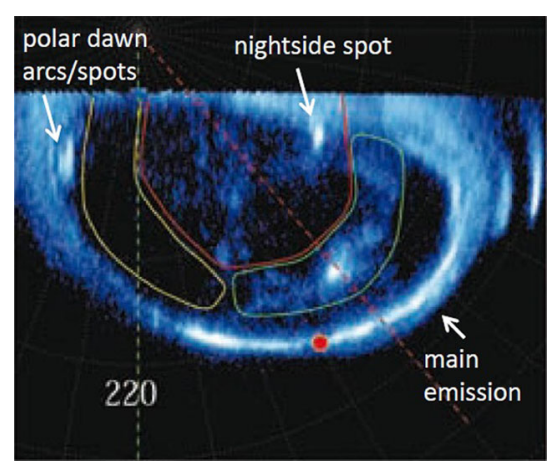

Fig. 10 Polar projection of the northern auroral region obtained from the Hubble Space Telescope on December 16, 2000, at CML $=220 \mathrm{deg}$. The main emission is indicated by the arrow. The shape and position of the three main polar regions are shown: the dark region (yellow contour), the swirl region (red contour), and the active region (green contour). The polar dawn arcs/spots and the nightside spot are indicated. Parallels and meridians are drawn every $10 \mathrm{deg}$. The CML is marked with a vertical green dashed line and longitude $180 \mathrm{deg}$ is highlighted with a red dashed line. The red dot locates the magnetic footprint of Ganymede (VIP4 model) as the orbital longitude of the satellite matches the CML and therefore indicates the direction of magnetic noon at $15 R_{J}$. Adapted from Grodent et al. (2003b)

At Saturn, observations of $\mathrm{H}_{3}^{+}$emissions in the infrared domain demonstrated the presence of an auroral oval mapping to a radial distance of $4 R_{S}$ (Stallard et al. 2008). This infrared secondary oval is thus located at lower latitude than the ultraviolet oval described above. The location and the faint emissions are consistant with models of a current system associated with the corotation breakdown of the magnetospheric plasma, similar to the Jupiter case (see also Ray et al. 2013).

\subsection{Dynamics of the Polar Aurora}

\subsubsection{Jupiter}

The emissions located poleward of the main emission at Jupiter, the polar emissions, are suggested to be magnetically connected to the middle and outer magnetosphere and possibly related to a sector of Dungey and/or Vasyliunas cycle flows (Cowley et al. 2003; Grodent et al. 2003b; Stallard et al. 2003). They are classified into three main regions: the swirl, the dark and the active region (Fig. 10).

The swirl region is the polar-most region of the polar emissions. It usually displays weak and very dynamic patches of emission that sometimes give the impression of a rotating crown on animations made of successive images. Its IR counterpart is the fixed- Dark Polar region (Stallard et al. 2003). According to the magnetic field mapping model by Vogt et al. (2011), this area is a region of open flux, which is consistent with the fact that the ionospheric flow are fixed relative to the sun, though Delamere and Bagenal (2010) argued that this region may be tied to intermittently open flux stemming from a viscous interaction along the dawn flank.

Polar auroral filaments have been observed in the same region. Nichols et al. (2009b) reported the finding of thin rectilinear and quasi-sun aligned features in the swirl region. The sunward head of the filaments remains fixed in local time while the anti-sun-ward part sub-corotates. These filaments were seen in $7 \%$ of the 2007 HST observations and their occurrence appears to be independent from the solar wind conditions. Nichols et al. (2009b) suggested that these emissions could be related to magnetotail dynamics. 
The dark region is usually devoid of significant UV emissions. It appears to correspond to a region that is also relatively dark in the IR: the rotating Polar Dark Region (r-DPR). This region has been associated with downward going field aligned currents, which would explain why the region is dark (Grodent et al. 2003b). However, the brightness of the IR emissions in the r-DPR still represent 30 to $40 \%$ of those of the main emissions relative brighter in IR compared to the low emission in UV (Stallard et al. 2003). Recent studies suggested that this difference is explained in terms of soft precipitation and most probable Joule heating (Radioti et al. 2013a). Moreover, the mapping model from Vogt et al. (2011) suggests that half of the dark region corresponds to closed field lines while the other half corresponds to opened field lines, in contradiction with the apparent uniformity of this region. Delamere and Bagenal (2013) suggested that this region contains closed flux with much of the missing flux from Vogt et al. (2011) stored in a wing along the dawn flank. The FUV-dark region also appears dark in the X-rays (Branduardi-Raymont et al. 2008).

At the equatorward edge of the dark region, at the dawn and midnight flank of the main emission auroral observations have shown the occasional appearance of spotty transient emissions (Fig. 10). In particular, parallel arc structures are observed to be located in the dawn sector (Grodent et al. 2003a) and isolated spots to appear in the dusk-midnight sector, poleward of the main emission (Grodent et al. 2004). The dawn arcs and the nightside spots were proposed to be triggered by reconnection processes in the jovian magnetotail, given their observed location and properties. An analysis based on daily UV auroral observations (Radioti et al. 2008b) revealed the presence of periodic auroral features ("polar dawn spots", similar to the dawn arcs (Grodent et al. 2003a). They consist of transient auroral emissions in the polar dawn region, with a characteristic recurrence period of 2-3 days. Because of their periodic recurrence and observed location, the polar dawn spots were interpreted as auroral signatures of internally driven magnetic reconnection in the jovian magnetotail (Vasyliunas cycle). Particularly, they were associated with the inward moving flow bursts released during magnetotail reconnection in Jupiter's tail (Radioti et al. 2010). The association of the polar dawn auroral spots with tail reconnection was further confirmed by Ge et al. (2010). The authors magnetically mapped tail reconnection events into Jupiter's ionosphere, by tracing field lines using a jovian magnetosphere model (Khurana 1997).

More recently, Radioti et al. (2011b) reported observations of a dusk side spot occurring at nearly the same time as a reconnection signature was observed in the Galileo magnetometer data (Vogt et al. 2010). This spot was mapped using an updated mapping model to an equatorial position close to the Galileo spacecraft and inside of a statistical X-line, further confirming the association of the auroral spots with inward flow from tail reconnection. Not only UV but also IR emissions bear the signature of tail reconnection. Comparison of nearsimultaneous UV and IR observations on 26 July 1998 has revealed a bright IR polar spot, which could be a possible signature of tail reconnection (Radioti et al. 2011b). The IR spot appears within an interval of 30 minutes from the ultraviolet, poleward of the main emission in the ionosphere and in the post-dusk sector planetward of the tail reconnection X-line in the equatorial plane. Finally, auroral observations can provide a hint as to the extent of the tail X-line. Near-simultaneous HST auroral and Galileo observations demonstrated that ionospheric signatures of inward moving flows released during tail reconnection are instantaneously detected over a wide local time range (Radioti et al. 2011b). However, whether reconnection at Jupiter's tail can result in a simultaneous release of flow bursts over a large local time sector is a question still to be resolved by future missions to Jupiter and/or remote observations.

The brightest polar region is called the active region and is located inside the dusk flank of the main emission. It usually displays a mix of quiet and dynamic auroral spots, blobs 
Fig. 11 Polar projection of

Saturn's northern aurora obtained with the FUV channel of UVIS onboard Cassini on January 21, 2009. Noon is to the bottom and dusk to the right. The grid shows latitudes at intervals of $10^{\circ}$ and meridians of $40^{\circ}$. Arrows indicate the main emission and bifurcations of the main emission. Adapted from Radioti et al. (2011a)

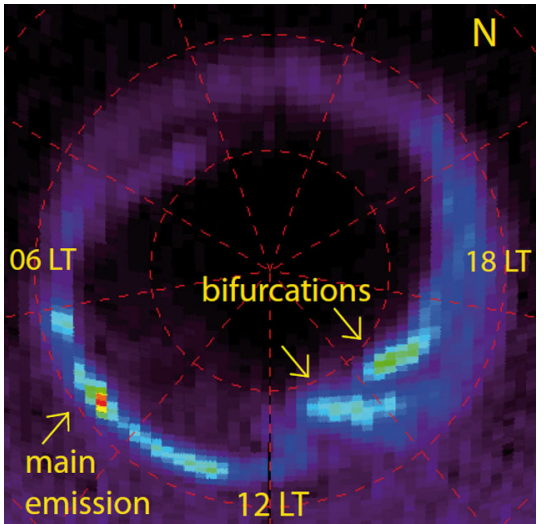

and arcs (Grodent et al. 2003b). It's IR counterpart appears to be the Bright Polar Region (Stallard et al. 2003).

In the active region, extremely bright flares are observed. These are localized transient features, which exhibit intense brightenings. Waite et al. (2001) reported an extreme case where the brightness reached $40 \mathrm{MR}$. Elsner et al. (2005) compared the timing and the location of the UV and the Xray flares and found that flare brightenings in both wavelengths were quasi-simultaneous, but not exactly co-located. Simultaneous UV and X-Ray observations demonstrated that the $<2 \mathrm{keV}$ photons appear co-located in the "active region", while there is absence of high energy X-ray photons in this area (Branduardi-Raymont et al. 2008). Finally, Bonfond et al. (2011) reported two cases of quasiperiodic variations of these flare emissions, with a re-occurrence time of 2-3 minutes. They also noted a rapid dawnward propagation of the flares in one of the cases. They tentatively attributed these periodic flares to a signature of pulsed component reconnection on the dayside magnetosphere. However, the recent finding of similar periodicities in high-energy electron data in the outer and middle magnetosphere put this interpretation into question. Moreover, Pallier and Prange (2001) suggested that some of the emissions in the active region could be associated with the cusp.

\subsubsection{Saturn}

Hubble Space Telescope (HST) observations demonstrated that Saturn's aurora responds to solar wind changes (Grodent et al. 2005; Clarke et al. 2009) and its brightness and shape varies with time. The main auroral emission at Saturn (Fig. 11) is suggested to be produced by the magnetosphere-solar wind interaction, through the shear in rotational flow across the open closed field line boundary (OCFLB) (e.g. Cowley and Bunce 2003; Bunce et al. 2008). Saturn's auroral morphology is, to a large extent, controlled by the balance between the magnetic field reconnection rate at the dayside magnetopause and the reconnection rate in the nightside tail (Cowley et al. 2004; Badman et al. 2005, 2014b). Recently, Ultraviolet Imaging Spectrograph (UVIS) observations revealed the presence of small-scale structures in the dayside main auroral emissions indicative of magnetopause Kelvin-Helmholtz instabilities (Grodent et al. 2011).

Observations (Gérard et al. 2004, 2005) and theoretical studies (Bunce et al. 2005a) showed that bright FUV emissions at Saturn observed occasionally near noon are probably associated with reconnection occurring at the dayside magnetopause, similar to the "lobe cusp spot" at Earth (i.e. emissions located at the cusp magnetic foot point, Fuselier et al. 2002). Specifically, it was proposed by Bunce et al. (2005a) that pulsed reconnection at the 
low-latitude dayside magnetopause for northward directed Interplanetary Magnetic Field (IMF) is giving rise to pulsed twin-vortical flows in the magnetosphere and ionosphere in the vicinity of the OCFLB. For the case of southward IMF and high-latitude lobe reconnection pulsed twin-vortical flows, bi-polar field-aligned currents are expected and associated with auroral intensifications poleward of the OCFLB. Recently, Cassini UVIS revealed the presence of bifurcations of the main dayside auroral emission, which are interpreted as signatures of consecutive reconnection events at Saturn's magnetopause (Radioti et al. 2011a). The authors suggested that magnetopause reconnection can lead to a significant increase of the open flux within a couple of days. In particular, it was estimated that each reconnection event opens $\sim 10 \%$ of the flux contained within the polar cap. Further studies based on Cassini multi-instrument observations, including auroral UV and IR data, confirmed that the auroral arcs are related to bursty reconnection at Saturn involving upward field aligned currents (Badman et al. 2012b) and suggested that bursty reconnection at Saturn is efficient at transporting flux (Badman et al. 2013). Additionally, auroral observations have shown evidence of magnetopause reconnection at multiple sites along the same magnetic flux tube similar to the terrestrial case (Fasel et al. 1993), which give rise to successive rebrightenings of auroral structures (Radioti et al. 2013c).

In addition to dayside reconnection, tail reconnection leaves its signature in the aurora at Saturn. Changes in open flux obtained from the auroral images and comparison with open flux estimated from the upstream interplanetary data allowed the estimation of the average tail reconnection rates at Saturn (Badman et al. 2005). Also, small spots of auroral emission lying near the main emission, observed by the UVIS instrument onboard Cassini, are suggested to be associated with dipolarizations in the tail (Jackman et al. 2013). These auroral features are suggested to be the precursor to a more intense activity associated with recurrent energization via particle injections from the tail following reconnection and plasmoid formation (Mitchell et al. 2009).

Auroral dawn enhancements expanding in the polar auroral region have been recently reported in different studies and related to tail reconnection. Nichols et al. (2014) presented HST auroral intensifications in the dawn auroral sector, propagating at $330 \%$ rigid corotation from near $01 \mathrm{~h} \mathrm{LT}$ toward $08 \mathrm{~h} \mathrm{LT}$. They suggested that these emissions are indicative of ongoing, bursty reconnection of lobe flux in the magnetotail, with flux closure rates of $280 \mathrm{kV}$. Similar events of intense dawn auroral activity were recently observed by the UVIS instrument on board Cassini (Radioti et al. 2014a) and was characterized by significant flux closure with a rate ranging from 200 to $1000 \mathrm{kV}$. Additionally, Radioti et al. (2014b) revealed multiple intensifications within an enhanced auroral dawn region suggesting an $x$-line in the tail, which extends from 02 to 05 LT. The localized enhancements evolved in arc and spot-like small scale features, which were suggested to be related to plasma flows enhanced from reconnection which diverge into multiple narrow channels then spread azimuthally and radially. They proposed that the evolution of tail reconnection at Saturn may be pictured by an ensemble of numerous narrow current wedges or that inward transport initiated in the reconnection region could be explained by multiple localized flow burst events. Badman (2014) reported on Saturn's auroral morphology during a solar wind compression event. The authors suggested that their observations were evidence of tail reconnection events, initially involving Vasyliunas-type reconnection of closed mass loaded magnetotail field lines and then proceeding onto open field lines, causing contraction of the polar cap region.

Enhancements in energetic neutral atom (ENA) emission and Saturn kilometric radiation (SKR) data, together with auroral observations from HST and UVIS reported the initiation of several acceleration events in the midnight to dawn quadrant at radial distances of 15 to $20 R_{S}$, related to tail reconnection (Mitchell et al. 2009). 
Fig. 12 Polar projection of Saturn's aurora as captured from UVIS onboard Cassini on DOY 224,2008 . The arrow indicates the first observation of Saturn's transpolar arc. Its formation is possibly related to tail reconnection (adapted from Radioti et al. 2014a)

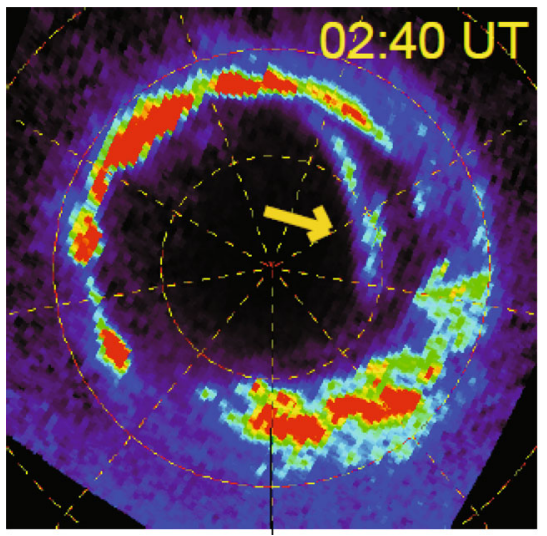

Finally, UVIS auroral observations revealed the first and only observation of an Earth-like transpolar arc at Saturn (Fig. 12) (Radioti et al. 2014a). Transpolar arcs are features which extend from the nightside auroral oval into the open magnetic field line region (polar cap) and they represent the optical signatures of magnetotail dynamics (e.g. Frank et al. 1982; Kullen 2000; Milan et al. 2005). The authors suggested that the formation of the transpolar arc at Saturn is possibly related to tail reconnection similarly to the terrestrial case (Milan et al. 2005). However, the rarity of the occurrence of the transpolar arc at Saturn indicates that the conditions for its formation are rarely met at the giant planet, contrary to the Earth.

\subsection{Solar Wind Influence}

\subsubsection{Jupiter}

It has long been known that Jupiter's radio emissions associated with auroral processes are controlled by conditions in the interplanetary medium incident on Jupiter's magnetosphere (Terasawa et al. 1978; Barrow et al. 1986; Zarka and Genova 1983; Genova et al. 1987; Bose and Bhattacharya 2003; Echer et al. 2010; Hess et al. 2012). The first evidence that Jupiter's auroras themselves are modulated by conditions in the interplanetary medium was reported by Baron et al. (1996), who analysed NASA IRTF observations of the planet's $\mathrm{H}_{3}^{+}$emissions in conjunction with Ulysses solar wind data over a $\sim 100$ day interval near the Jupiter encounter in 1992. They showed that the change in solar wind dynamic pressure between their auroral observations was reasonably well correlated with the observed total intensity of the $\mathrm{H}_{3}^{+}$auroral emission. More detailed understanding was obtained during the Cassini Jupiter flyby in late 2000-early 2001. A combination of Cassini and Galileo spacecraft in situ measurements and remote sensing, along with a program of Hubble Space Telescope (HST) Space Telescope Imaging Spectrograph (STIS) observations near to closest approach revealed in detail the response of the auroral emissions to changing conditions in the interplanetary medium (Gurnett et al. 2002; Pryor et al. 2005; Nichols et al. 2007). The solar wind during the interval of the encounter, which occurred at solar maximum, was dominated by coronal mass ejections (CMEs), corotating interaction regions (CIRs) and large amplitude stream interactions resulting in deep several-day solar wind rarefaction regions punctuated by strong compressions as highlighted by the interplanetary magnetic field (IMF) magnitude shown in Fig. 13(a), taken from Gurnett et al. (2002). These authors showed that, when 
Fig. 13 Plot showing interplanetary medium, radio and EUV data from (a) the Cassini MAG instrument, (b) the solar wind ion densities from the Cassini CAPS instrument, (c) the integrated $(0.5 \pm 5.6 \mathrm{MHz})$ hectometric radiation intensities (1-h averages) from the Galileo PWS instrument, and (d) the disk-integrated extreme ultraviolet auroral $\mathrm{H}_{2}$ band $(110.8 \pm 113.1 \mathrm{~nm})$ intensities for the Cassini ultraviolet imaging spectrograph (UVIS) instrument for event $\mathrm{A}$. The arrows show the times at which the interplanetary shock was detected by Cassini and Galileo. Reproduced from Gurnett et al. (2002)

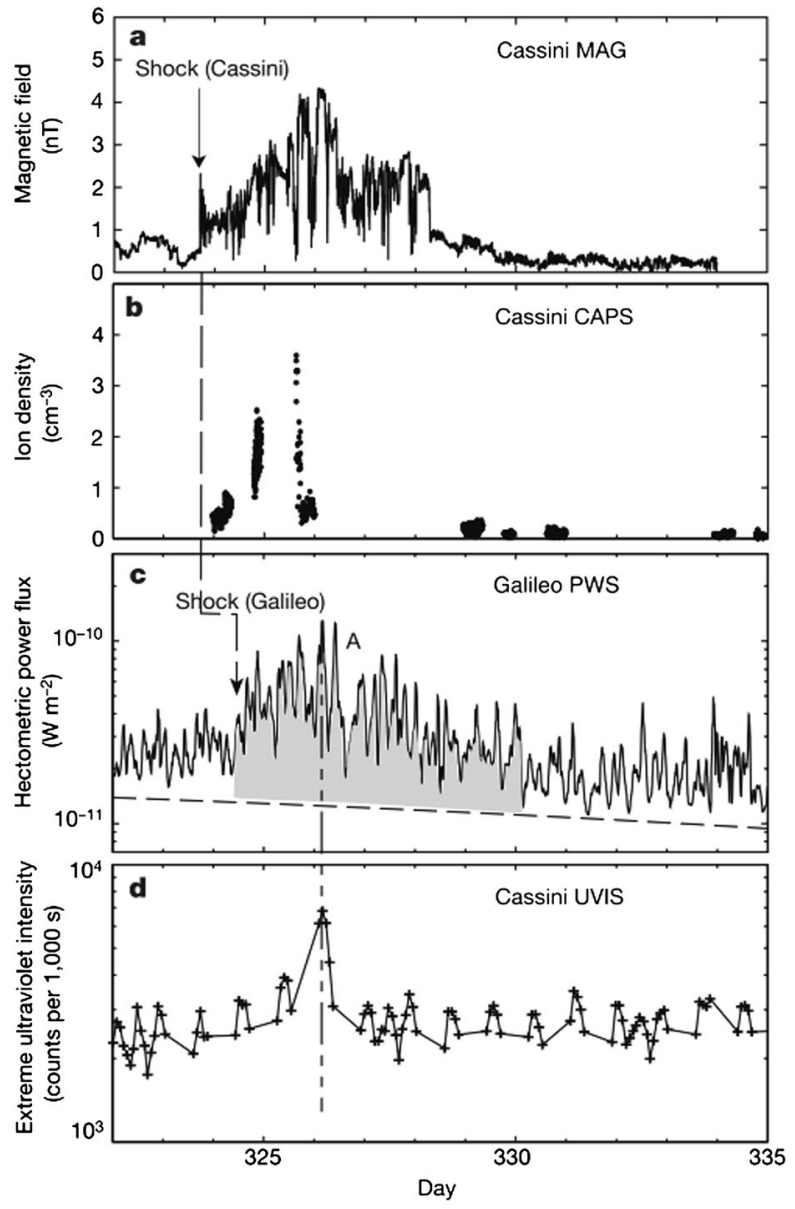

corrected for solar wind travel time, the jovian extreme ultraviolet (EUV) emission and hectometric radiation exhibited peaks in intensity of factors of 2-4 above the background near to the time of maximum solar wind density as shown in Figs. 13(b-c).

While the global response of the auroral intensity to the interplanetary conditions was thus revealed by the Cassini EUV data, the detailed morphological variation was elucidated by the contemporaneous HST Space Telescope Imaging Spectrometer (STIS) images of the far ultraviolet (FUV) aurora discussed by Grodent et al. (2003a,b) and Nichols et al. (2007). The conditions in the interplanetary medium observed at the time of HST observations, suitably propagated from the Cassini spacecraft to the planet's ionosphere, are shown in Fig. 14, adapted from Nichols et al. (2007). The plot is colour-coded such that red data points indicate solar wind, blue represents magnetosheath, and green signifies magnetosphere. The vertical grey lines indicate the times of the HST images. It is apparent that most of the HST images were obtained during solar wind rarefaction regions with dynamic pressure of order $\sim 0.01 \mathrm{nPa}$, corresponding to an expanded magnetosphere with a sub-solar distance of $\sim 70-100 R_{J}$. Only one visit on 13 January corresponds to a compression event with dynamic pressure $>0.1 \mathrm{nPa}$ and a correspondingly compressed magnetosphere of sub-solar extent $\sim 50-60 R_{J}$. In Fig. 14 we then also show two representative images of the north- 


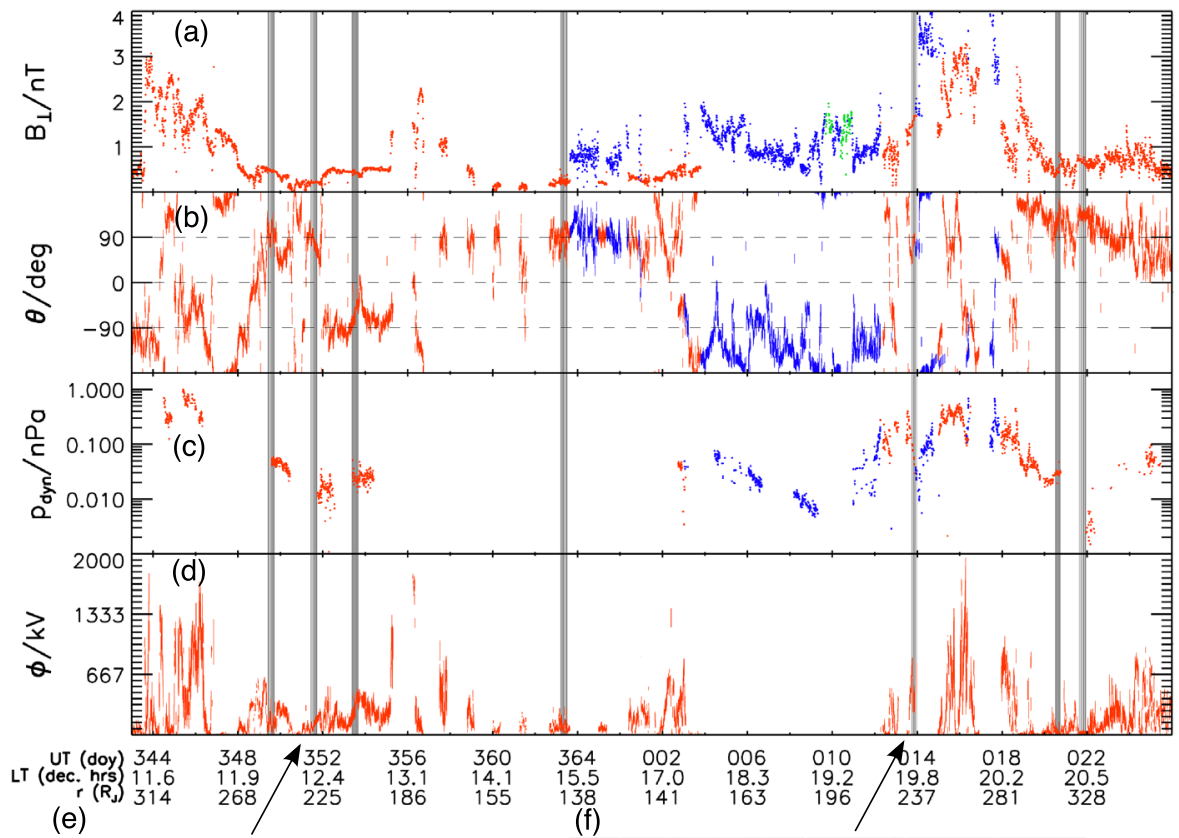

(e)
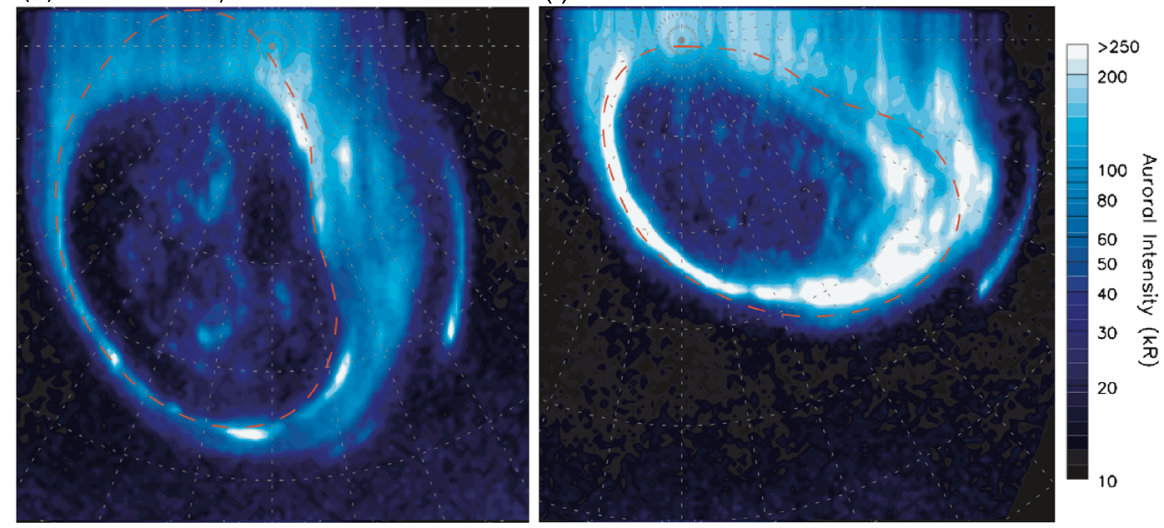

Fig. 14 Plot showing the conditions in the interplanetary medium as measured by Cassini over days $343 / 2000$ to $025 / 2001$, along with representative HST images of Jupiter's auroras obtained during the interval. From top to bottom the panels show: (a) the perpendicular IMF magnitude $B_{\perp}$ in nT, (b) the IMF clock angle relative to Jupiterôs spin axis $\theta$ in degrees, $(\mathbf{c})$ the dynamic pressure of the solar wind $p_{\text {dyn }}$ in $\mathrm{nPa}$ on a log scale, (d) and the estimated dayside reconnection voltage $\phi$ in $\mathrm{kV}$ calculated using the algorithm of Nichols et al. (2006). Data are plotted versus estimated time of impact on Jupiterôs ionosphere, along with the local time and range of Cassini relative to Jupiter. In the plots for $\theta$ and $\phi$ each 'data point' is stretched into a vertical line representing the effect produced by the $\sim \pm 9.5 \mathrm{deg}$ diurnal variation of Jupiterôs dipole axis offset. The vertical grey lines indicate the times of emission of the auroral photons observed by HST. Panels (e) and (f) show representative images of Jupiterôs northern UV aurora with CML obtained by the HST during the millennium campaign, projected onto a latitude-longitude grid viewed from above the north pole. The CML of each image, i.e., approximately the sunward direction, is aligned toward the bottom of each image, such that dawn is to the left, dusk to the right, and midnight to the top. The intensity scale is logarithmic and saturated at $250 \mathrm{kR}$. The Grodent et al. (2003a) reference main oval is shown by the dashed red line. A $10^{\circ} \times 10^{\circ}$ jovigraphic grid is overlaid. The images were obtained on 351/2000 and 013/2001. Adapted from Nichols et al. (2007) 
ern auroras (the hemisphere most visible from Earth due to the non-dipolar nature of the jovian internal magnetic field), corresponding to rarefaction and compression solar wind conditions.

The basic morphology of Jupiter's auroras has been discussed in Sects. 4.1 and 4.2, such that here we concentrate only on the differences between the two solar wind states. The auroras associated with the rarefaction region shown in Fig. 14(e) exhibit the 'classic' morphology, i.e. the main oval is narrow and well-defined between System III (SIII) longitudes $\sim 160-240^{\circ}$, typically lying in the dawn to noon sector at the central meridian longitudes for which the northern auroras are observed, while it is broad and diffuse in the 'kink' sector at smaller SIII longitudes. Poleward of the main oval lies a relatively dark region, more typically existing on the dawn side but occasionally extending around to the dusk side, and poleward still lies a region of patchy, variable emission which is particularly bright in an active region near noon. The images obtained during the compression region on 13 January, shown in Fig. 14(f), were in comparison obtained at high CML value, such that the viewing angle is less optimal than for the rarefaction regions, allowing good views only of the narrow main oval region. However, it is obvious that the main oval is significantly brighter and expanded poleward along its entire length, merging with bright auroral forms in the active region, and the polar patchy emission is also extended equatorward down to the main oval. The enhanced auroral power associated with the compression was thus a result of brightened and/or expanded main oval and polar emissions. It should be noted that, although these enhanced auroras were overall associated with a compression region, Nichols et al. (2007) noted that there was significant variation in the interplanetary conditions during this interval, such that it remains ambiguous as to whether the brightening was associated with a transient compression or expansion of the magnetosphere.

Further understanding of the response of Jupiter's auroras was obtained in 2007, when Jupiter's auroras were observed once per day using the Advanced Camera for Surveys (ACS) onboard HST for two month-long programs, the first in February/March during the New Horizons flyby and the second in May/June near opposition (Clarke et al. 2009; Nichols et al. 2009a). Using these data Clarke et al. (2009) considered the variation in total emitted FUV power in comparison to changes in the solar wind conditions as propagated from Earth using the 1-D MHD model of Zieger and Hansen (2008). In total, the two intervals included six solar wind forward shocks and three reverse shocks, the former associated with sharp increases in dynamic pressure occurring at compression region onset but which may also occur within larger merged compression regions, while the latter corresponds to sharp decreases in dynamic pressure at the tail end of compressions. With some uncertainty in the timing of the modelled propagation, Clarke et al. (2009) showed that forward shocks were typically associated with increases in total auroral power, while reverse shocks did not induce any significant change in auroral output. The variation of the individual components of auroral emission over these intervals was considered by Nichols et al. (2009a), from whose study some representative results are displayed in Figs. 15 and 16. Specifically, Figs. 15 $(\mathrm{a}-\mathrm{c})$ show the power variation from the 3 different regions of the auroras delimited by the solid yellow lines in Fig. 16, i.e. the high latitude region, the main oval, and the low latitude region, and panels (d) and (e) show the solar wind dynamic pressure and IMF magnitude estimated using the model of Zieger and Hansen (2008). In the bottom two panels the solid lines show the model result with the original timings, while the dotted lines show the results shifted by +2.1 days, calibrated to an interplanetary forward shock observed by the New Horizons spacecraft on day 53 (Clarke et al. 2009). The dark grey regions show the estimated arrival time of the forward shocks within $1-\sigma$ uncertainty of the MHD model timings, and the light grey regions are similar but for the shifted timings. 


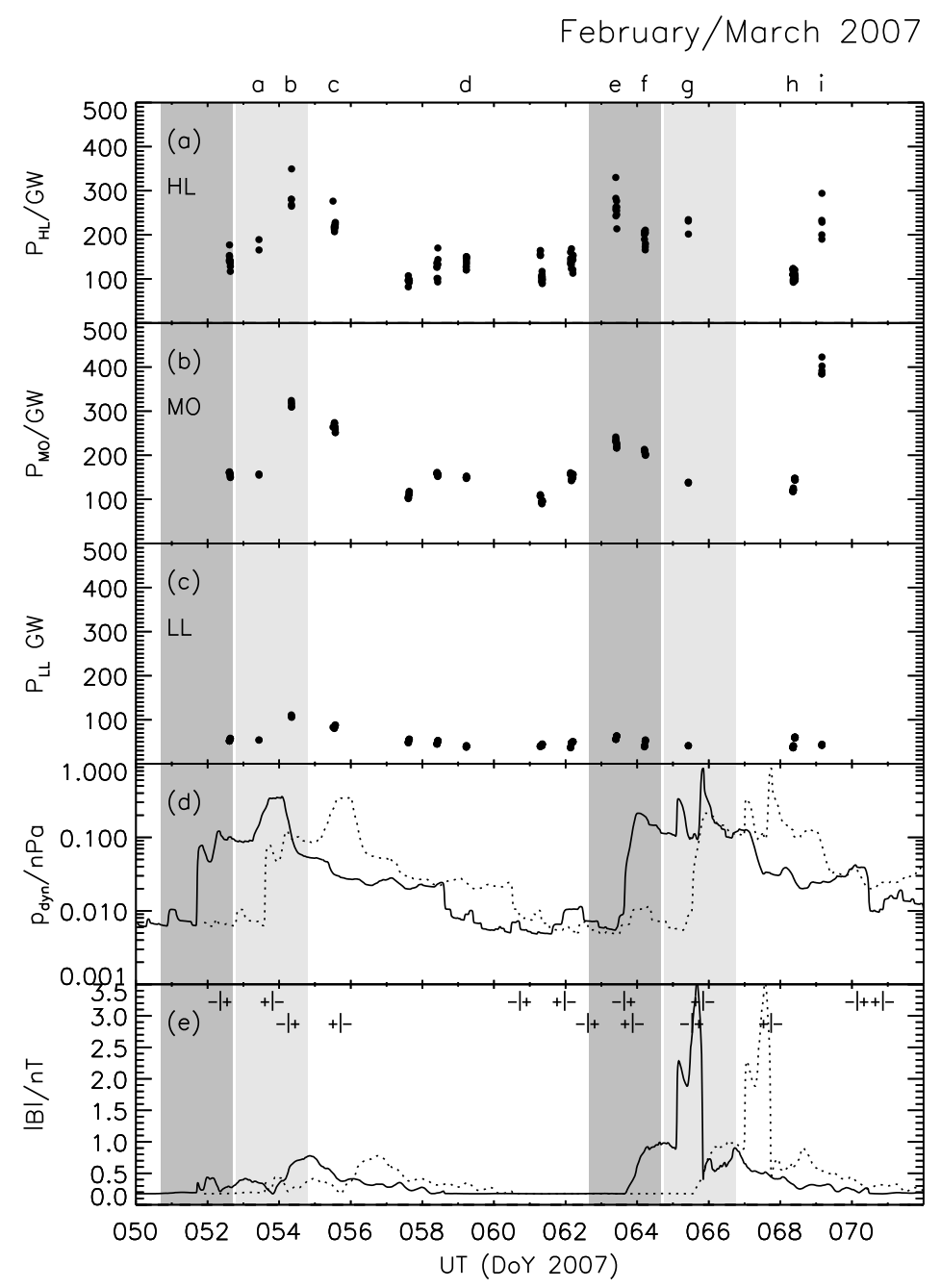

Fig. 15 Plots showing the power emitted from the different auroral regions, along with the modelled solar wind conditions for the first HST campaign in February/March 2007. Specifically, we show (a) the power emitted from the high latitude region $P_{H L}$ in $\mathrm{GW}$, (b) the power emitted from the main oval region $P_{M O}$ in GW, (c) the power emitted from the low latitude region $P_{L L}$ in $\mathrm{GW}$, (d) the solar wind dynamic pressure in $\mathrm{nPa}$, and (e) the IMF magnitude $|B|$ in nT. The individual points in panels (a)-(c) represent the powers obtained for each image. The solid lines in the MHD model panels show the original model timings, while the dotted line show the timings shifted by +2.1 days. The dark grey regions shows the estimated arrival time of the forward shocks within 1 standard deviation uncertainty of the MHD model timings, and the light grey regions are similar but for the shifted timings. Also shown in panel (e) are the estimated locations of the heliospheric sector boundaries, along with the sign of $B_{T}$ either side. The original timing is on top, while the shifted timing is below. Reproduced from Nichols et al. (2009a)

As discussed by Clarke et al. (2009), three overall brightness enhancements were observed, two corresponding forward shocks (the first using the shifted model timings, the second using the original timing), while the third, a dawn storm, does not correspond obviously to any solar wind event. The auroral emissions corresponding to the data points labelled at the 


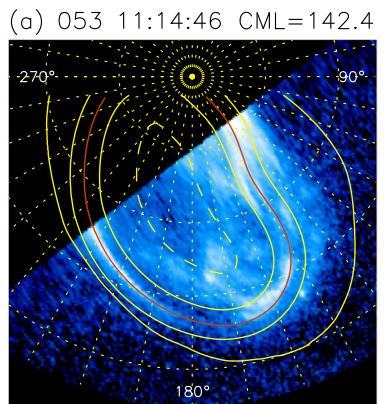

(d) $059 \quad 06: 16: 20 \quad \mathrm{CML}=144.7$

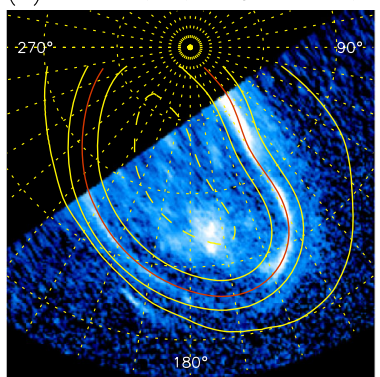

(g) 065 11:00:46 $\mathrm{CML}=140$

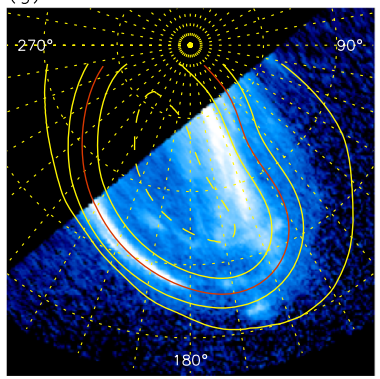

(b) 054 08:55:53 $\mathrm{CML}=209.0$

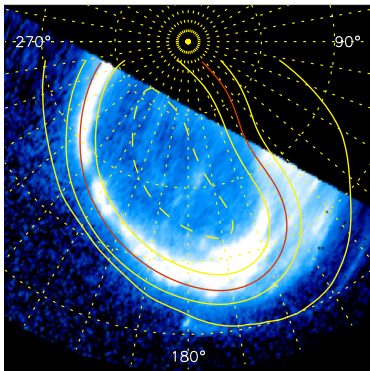

(e) $063 \quad 10: 23: 41 \quad \mathrm{CML}=176.6$

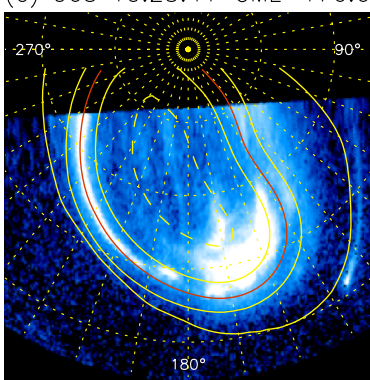

(h) $068 \quad 09: 19: 29 \quad \mathrm{CML}=170.1$

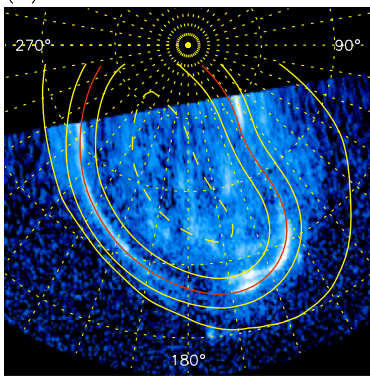

(c) 055 13:51:47 $\quad \mathrm{CML}=178.4$

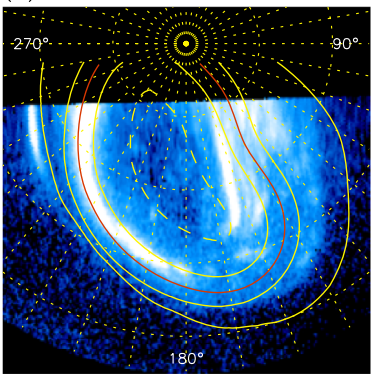

(f) $064 \quad 06: 13: 19 \quad C M L=175.8$

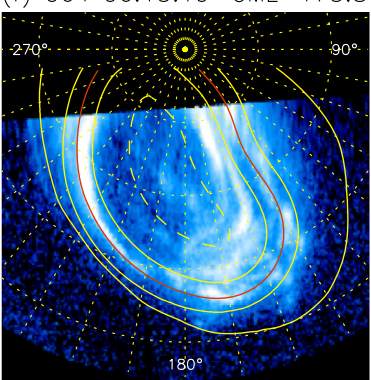

(i) $069 \quad 04: 38: 37 \quad \mathrm{CML}=151.2$

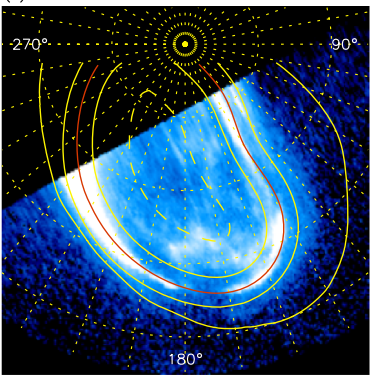

Fig. 16 Representative HST images of Jupiter's northern auroras corresponding to the visits labeled at the top of Fig. 15. The projection view is from above the north pole, and the image is displayed with a log color scale saturated at $500 \mathrm{kR}$. The red line shows the reference main oval as given by Table 1 in Nichols et al. (2009a). The solid yellow lines show the boundaries between the high latitude region, the main oval and the low latitude emission. The dashed yellow line indicates the boundary between the polar inner and polar outer regions. The yellow points indicate a $10^{\circ} \times 10^{\circ}$ planetocentric latitude-SIII longitude grid. The image is oriented such that SIII longitude $180^{\circ}$ is directed toward the bottom. Reproduced from Nichols et al. (2009a)

top of Fig. 15(a) are shown in Fig. 16. Using these data, Nichols et al. (2009a) showed that Jupiter's auroras respond to solar wind compression region onset in a broadly repeatable manner, which can be summarised as follows:

- The total emitted power from the main oval increases by factors of $\sim 2-3$.

- The main oval is brightened along longitudes $>165^{\circ}$, and is shifted poleward by $\sim 1^{\circ}$ and expanded, as evidenced in Figs. 16(b) and (c), and (e-g).

- In contrast, there is little emission at longitudes $<165^{\circ}$, and any auroras are patchy and disordered.

- Under-sampling notwithstanding, the main oval apparently persists in this disturbed state for 2-3 days following compression region onset. 
- The poleward emission varies broadly with the main oval, but with significant variation superposed thereon.

- The noon active region is brightened for $\sim 1$ day and merges with the poleward-shifted main oval, as shown in Figs. 16(b) and (e)

- Bright poleward dusk arcs, sometimes multiple in nature, are apparent for $\sim 2$ days following compression region onset, as evidenced in Figs. 16(c) and (f).

- The high latitude patchy auroras vary independently from the lower latitude polar auroras and the main oval, causing the superposed variation mentioned above.

- Dawn storms, for which the main characteristic is exceptionally bright dawn-side main oval auroras, but in which all auroral components brighten simultaneously as shown in Fig. 16(i), occur sporadically with no apparent solar wind trigger.

As well as these changes in the morphology of Jupiter's auroras in response to varying conditions in the interplanetary medium, there are a number of auroral forms which exhibit local time alignment or are thought to be associated with the solar wind interaction. The active region located near noon has been observed to 'flare' from a few kR to $\sim 10 \mathrm{MR}$ over a few minutes (Waite et al. 2001), and transient 'inner ovals' a few degrees poleward of the main oval have also been reported (Ballester et al. 1996; Pallier and Prange 2001; Nichols et al. 2007), similar to those thought to exist at the open-closed field line boundary (Cowley et al. 2005a, 2007). It has been suggested that at least some of the polar auroras may be related to the solar wind interaction at the dayside (Clarke et al. 1998; Pallier and Prange 2001; Waite et al. 2001; Grodent et al. 2003b). This idea was explored theoretically by Bunce et al. (2004), who showed that pulsed dayside reconnection with a $\sim 45$ min period during intervals of strong solar wind interaction could excite adjacent high latitude regions of UV and X-ray emission, as observed by Gladstone et al. (2002) and Elsner et al. (2005). Pulsed reconnection is also postulated to be responsible for periodic polar flares observed in the southern auroras, which exhibit a 2-3 minute periodicity and propagate swiftly from dusk to dawn (Bonfond et al. 2011). Radioti et al. (2008b) presented observations of periodic polar dawn spots of auroral emission just poleward of the main oval, which exhibit a 2-3 day periodicity, and were attributed to nightside reconnection, while Radioti et al. (2008a) showed that the main oval exhibits a persistent dim region ('discontinuity') in the pre-noon sector thought to represent a decrease in field-aligned current intensity owing to increased equatorial plasma angular velocity due to confinement by the magnetopause. Quasi-sun-aligned polar auroral filaments were also reported by Nichols et al. (2009b), which are superficially similar to terrestrial trans-polar arcs but probably not generated in the same manner. They were observed over an interval of 6 days in 2007 and appear independent of incident solar wind conditions, and were thus postulated to map significantly down the tail. The origin of this feature is unknown and it is hoped that the Juno and JUICE spacecraft will shed light on Jupiter's enigmatic polar auroras.

\subsubsection{Saturn}

As for Jupiter, the modulation of Saturn's auroral current system by the incident solar wind was first recognized through observations of the associated radio emissions; Voyager observations showed that the intensity of the Saturn Kilometric Radiation (SKR) was correlated with solar wind dynamic pressure (Desch and Kaiser 1981). The UV spectrometer onboard Voyager also revealed auroral emissions at a latitude consistent with the open-closed fieldline boundary (Sandel and Broadfoot 1981). The detailed morphology of Saturn's auroras was revealed by later HST images, with Badman et al. (2006) and Bunce et al. (2008) confirming the co-location with the open-closed field-line boundary and Gérard et al. (2005) 
Fig. 17 Comparison between HST images and solar wind conditions propagated to Saturn for the period 2530 January 2004. Reproduced from Crary et al. (2005)

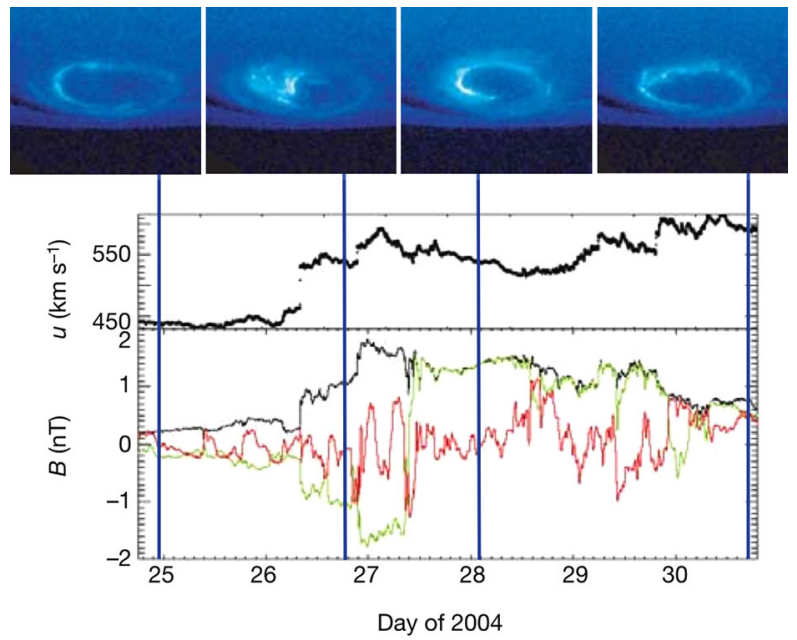

identifying a transient noon auroral feature on the poleward edge of the main oval with the cusp. Transient duskside poleward forms observed in the south were associated by Radioti et al. (2009b) with energetic particle injections, although later analysis of similar features by Meredith et al. (2013), who examined equinoctial HST images of Saturn's simultaneous conjugate auroras, showed them to be hemispherically asymmetric and thus ascribed them to dayside reconnection of the rotating planetary field with $B_{y}$-dominated IMF.

The breakthrough in understanding the major effect of the solar wind on Saturn's auroras occurred as the Cassini spacecraft was approaching the planet in January 2004, and a large CIR passed over the spacecraft, corresponding to significantly increased solar wind velocity, dynamic pressure and IMF magnitude as shown in Fig. 17. As also shown in the figure, contemporaneous HST/STIS images of Saturn's southern auroras revealed that in response to these enhancements the dawnside auroras brightened significantly and expanded poleward, filling the dawnside polar cap with emission, while the radius of the oval shrank in proportion to the brightness (Crary et al. 2005; Clarke et al. 2005). Using the large HST/ACS program in 2007/2008, this was shown by Clarke et al. (2009) to be a repeatable morphological response to solar wind compression region onset, with the total power typically increasing by factors of $\sim 2-3$, as shown in Figs. 18 and 19. Further analysis of the SKR intensity over an extended interval has also revealed a positive correlation with solar wind pressure (Rucker et al. 2008). This morphology has been interpreted theoretically as a manifestation of compression-induced tail reconnection (Cowley et al. 2005b), while the effect of IMF direction has also been shown to be a significant factor in controlling the radius of the auroral oval (Belenkaya et al. 2010, 2011).

Since orbit insertion, the Cassini spacecraft has yielded significant information regarding the solar wind effect on Saturn's auroras. The UV Imaging Spectrometer (UVIS) and Visible and Infrared Mapping Spectrometer (VIMS) onboard Cassini have also revealed details of small-scale features in Saturn's auroras, some of which are associated with the solar wind interaction. Grodent et al. (2011) showed that Saturn's main oval comprises individual auroral patches, which they associated with the Kelvin-Helmholtz instability, although Meredith et al. (2013) later showed that, rather than being conjugate, patches in the two hemispheres are magnetically interlaced, and therefore identified them instead with the field-aligned currents of eastward-propagating ULF waves. Radioti et al. (2011b) analysed bifurcations in the main oval, and associated these with pulsed dayside reconnection, 

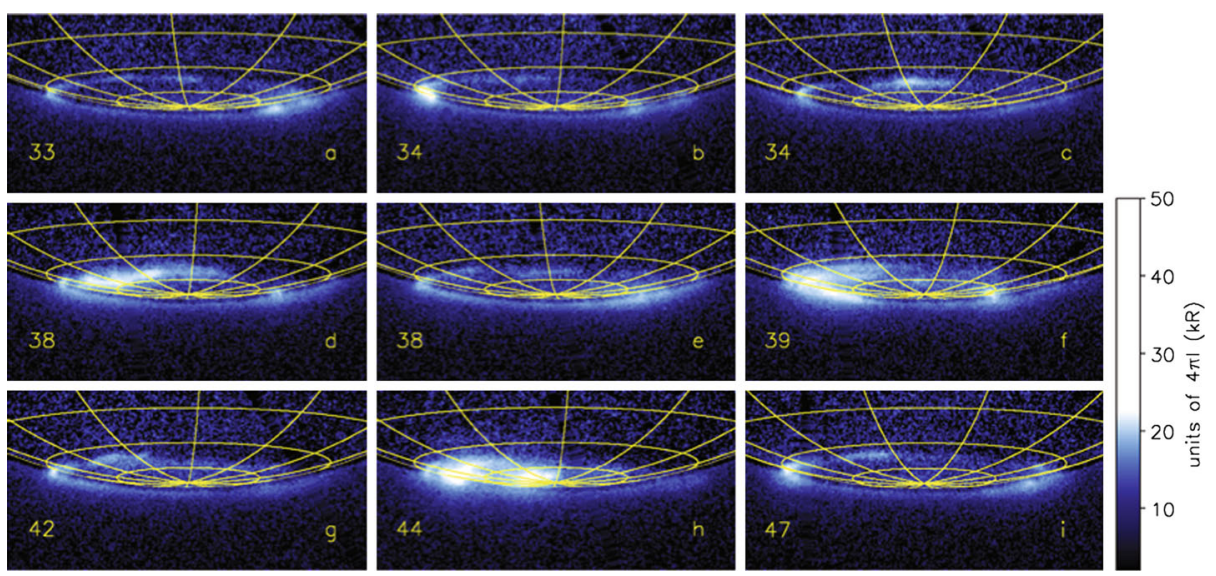

Fig. 18 Sample UV images of Saturnôs south pole in February 2008 with quiet and disturbed conditions. The left-hand number is day of year in 2008, and the part label letters correspond to the lettering at the top of Fig. 19. All frames were obtained with ACS, with a limiting sensitivity of 1 to $2 \mathrm{kR}$ after modelling and subtraction of reflected solar emissions. Reproduced from Clarke et al. (2009)

while Badman et al. (2012b) identified small-scale equatorward moving $\mathrm{H}_{3}^{+}$auroral forms, as shown in Fig. 20, with dayside reconnection although it was unclear as to whether the reconnection was at high- or low-latitudes. Recently, Badman et al. (2013) have presented observations of poleward features near noon, in conjunction with in situ evidence of recent dayside reconnection with the $B_{y}$-dominated IMF. Overall, therefore a considerable body of evidence has now been built up suggesting the solar wind plays important roles, both directly and indirectly, in modulating the auroral morphology at the solar system's two giant planets.

\section{Global Modeling of the Giant Planet Magnetospheres}

\subsection{Global Modeling Techniques and Limitations}

The most commonly used models in simulating planetary magnetospheres are magnetohydrodynamic (MHD) models in which the plasma is treated as a magnetized fluid. While an MHD model does not treat plasma kinetic effects (e.g. gradient/curvature drift and microscale wave-particle interactions) and the small-scale processes (e.g. reconnection) are facilitated by numerical resistivity, it has been shown to be capable of providing a reasonably good description of the large-scale structure and dynamics of planetary magnetospheres. Moreover, because MHD models can usually cover a large-size simulation domain and, at the same time, achieve reasonably high spatial resolution at relatively low computational costs (compared to kinetic/particle codes), it remains, at present, the most feasible tool for global simulations of planetary magnetospheres. This is especially the case for the gas giants, Jupiter and Saturn, because of the vast spatial and long temporal scales involved in the two magnetospheric systems.

There have been a number of global MHD models applied to modeling the magnetospheres of Jupiter (e.g. Ogino et al. 1998; Walker and Ogino 2003; Moriguchi et al. 2008; Fukazawa et al. 2005, 2006, 2010) and Saturn (e.g., Hansen et al. 2005; Fukazawa et al. 
Fig. 19 Total auroral power from Saturn's south polar region, best fit auroral oval radius, and SKR emission spectrum compared with propagated solar wind velocity and dynamic pressure in February 2008. Solar wind values obtained by propagation from Earth-based measurements have arrival times shifted 2.6 days later to match the time when Cassini measured a strong compression of the magnetosphere on DOY 38. Oval radius values were obtained by fitting a circle to the low-latitude edge of the observed auroral emissions. SKR emission measurements are from the Cassini RPWS instrument. Solar wind values were obtained by propagation from Earth-based measurements, with forward shock times $\pm 1 \sigma$ uncertainties shaded. Reproduced from Clarke et al. (2009)

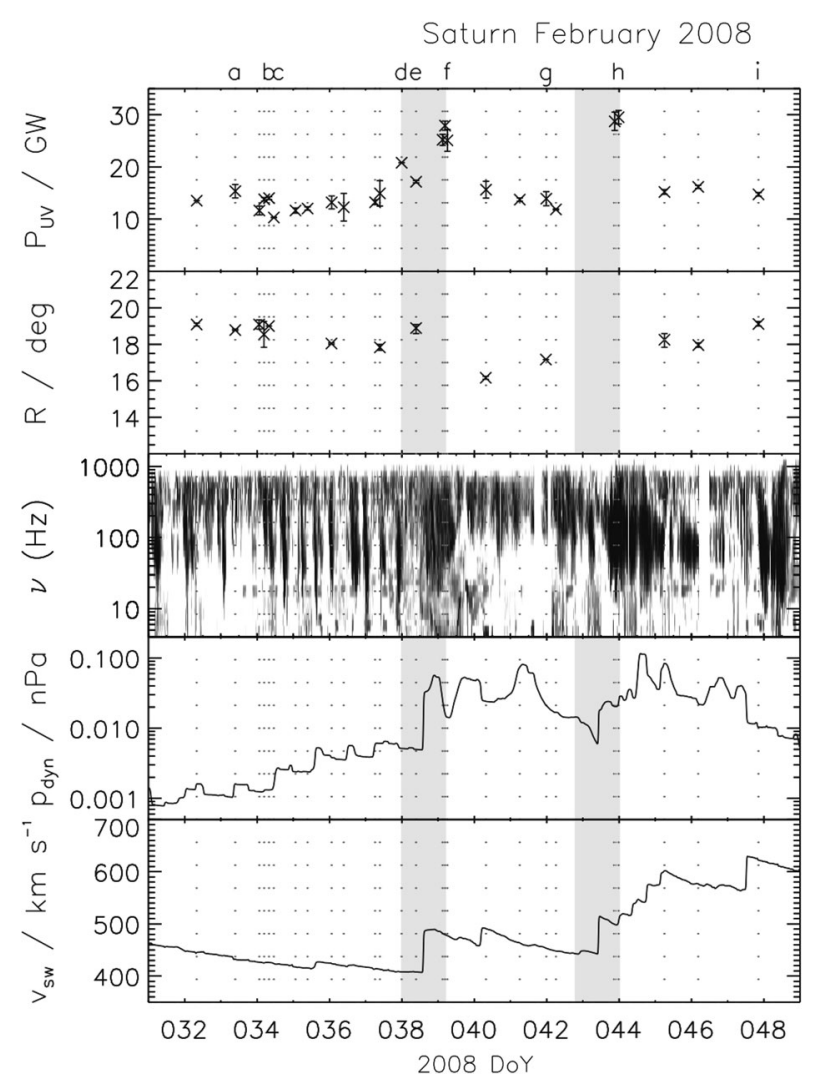

2007a,b; Kidder et al. 2012; Zieger et al. 2010; Walker et al. 2011; Jia and Kivelson 2012; Jia et al. 2012a,b; Winglee et al. 2013). In developing a global magnetosphere model for the giant planets, two aspects that are of particular importance are the rapid planetary rotation and strong internal plasma sources associated with the moons (in particular, Io in the case of Jupiter, and, Enceladus in the case of Saturn). All the global MHD models applied to the gas giants are designed to include these important factors to some extent, although they differ in many aspects, such as the assumption about the internal plasma sources, simulation boundary conditions, and the way of modeling the coupling between the magnetosphere and ionosphere. In the following, we provide an overview of the various global MHD simulations of the giant planet magnetospheres and highlight some important findings from global MHD models regarding the magnetospheric configuration and responses to solar wind driving.

\subsection{Global Modeling Results}

\subsubsection{Global Configuration}

Because of the rapid planetary rotation and strong internal plasma sources, the configurations of Jupiter's and Saturn's magnetospheres differ from that of a solar wind driven magnetosphere, such as the Earth's magnetosphere, where such effects do not play a significant role in shaping the magnetosphere under normal circumstances. There have been considerable efforts devoted to constructing empirical models of the magnetospheric boundaries 
(o)

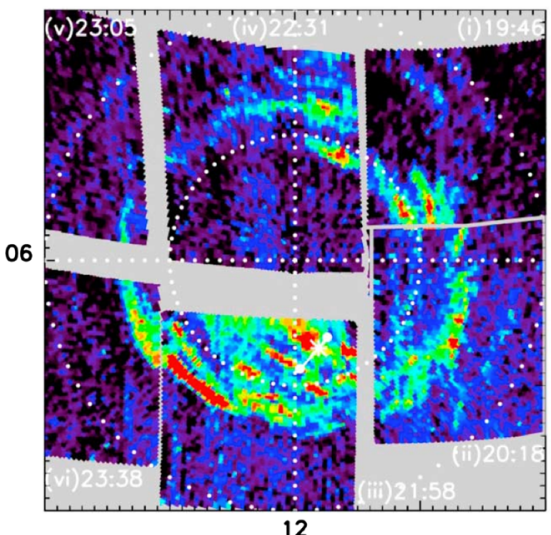

(b)

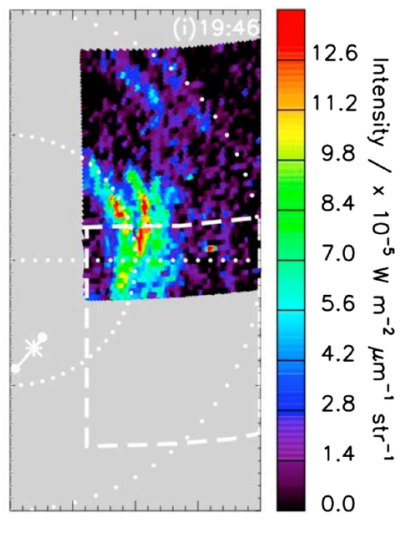

Fig. 20 (a) A mosaic of six Cassini VIMS images of Saturn's infrared aurora taken on 2008-320. The start time of each image is marked at its edge: (i) 19:46 UT, (ii) 20:18 UT, (iii) 21:58 UT, (iv) 22:31 UT, (v) 23:05 UT and (vi) 23:38 UT. The white grid marks latitudes at intervals of $10^{\circ}$ and the noon-midnight and dawn-dusk meridians. The white line delimited by dots shows Cassiniôs ionospheric footprint during 12:0024:00 UT on 2008-320. The white asterisk marks Cassiniôs footprint at 22:00 UT on DOY 320. (b) Image $\mathrm{i}$ taken by Cassini VIMS showing the area overlapped by the following image ii whose outline is marked by the white dashed line. Cassiniôs ionospheric footprint is shown here for clarity. Reproduced from Badman et al. (2012b)

based on in-situ observations. Complementary to those data-based empirical models, global MHD models of the giant planets that incorporate the aforementioned important effects may provide useful, quantitative information about the global shape of the magnetosphere as well as how it varies with both the external and internal conditions.

In a series of global simulation studies of Jupiter's magnetosphere (Ogino et al. 1998; Walker et al. 2005; Fukazawa et al. 2006), the authors have examined the modeled locations of Jupiter's magnetopause and bow shock for cases with and without the planetary rotation included, and also compared model results for low and high solar wind dynamic pressure conditions. By comparing the magnetopause locations from simulation runs with and without rotation included, their model results clearly demonstrate the effect of centrifugal inflation of Jupiter's magnetosphere (e.g., Hill et al. 1974). They also find that for the range of dynamic pressures used in their simulations, the modeled magnetopause locations, in general, fall within the range inferred from spacecraft observations.

Similar modeling studies on magnetospheric boundaries have been carried out for Saturn. For example, Jia et al. (2012b) have compared the standoff distances of Saturn's bow shock and magnetopause between their global MHD model and data-based empirical model. The non-steady solar wind input used in the Jia et al. MHD simulation allows for comparison for a wide range of solar wind dynamic pressures as well as for a variety of IMF conditions. As shown in Fig. 21, the sub-solar magnetopause location predicted by the MHD model agrees well with the prediction by the empirical model of Kanani et al. (2010) developed using Cassini data. For various external conditions considered, not only the mean location but also the variation of the magnetopause location show good agreement between the two models. The MHD model result also confirms the finding obtained from earlier analysis using Cassini data (e.g., Arridge et al. 2006; Kanani et al. 2010) that Saturn's magnetopause is neither as rigid as the Earth's nor as compressible as Jupiter's. An interesting feature predicted by both the MHD and empirical models is that as the dynamic pressure becomes weaker, Saturn's 

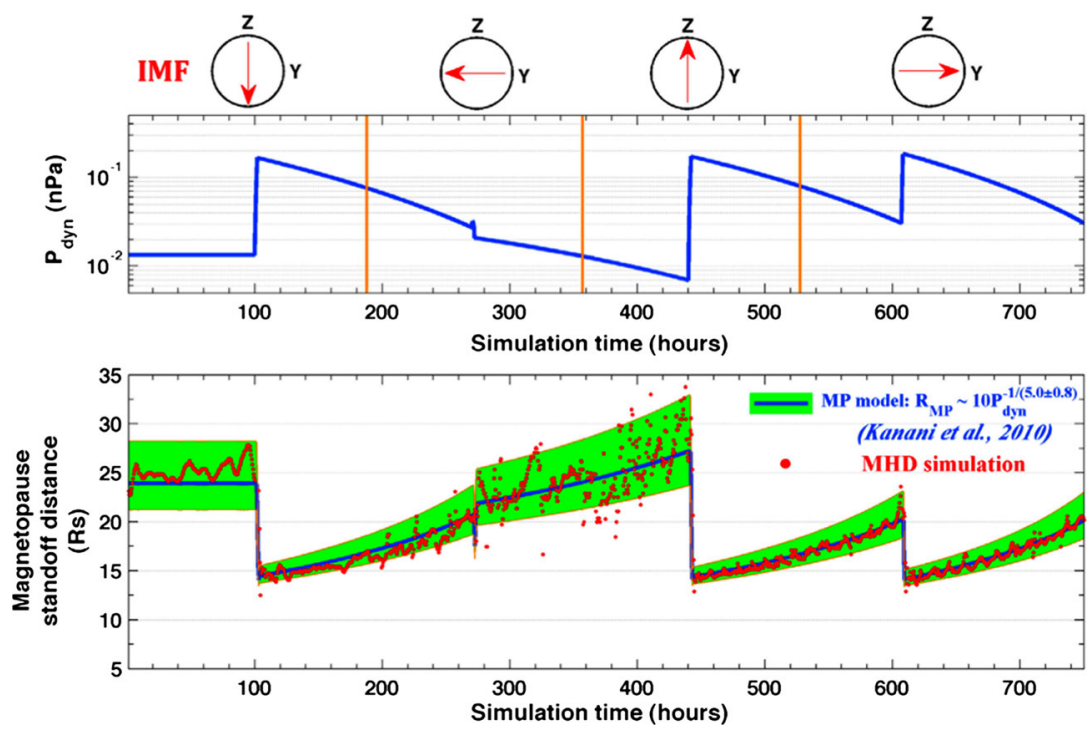

Fig. 21 Comparison of the magnetopause standoff distance between the global MHD simulation of Jia et al. (2012a) and the data-based empirical model of Kanani et al. (2010) constructed based on Cassini observations. (top) The solar wind conditions used as model input, including the IMF orientation (indicated by the red arrows) and the dynamic pressure. The orange lines mark the times when the discontinuities of IMF rotation arrive at the nose of the magnetopause. (bottom) The red dots show the standoff distance extracted from the MHD simulation and the blue trace along with the green shaded area indicate the standoff distance predicted by the magnetopause model of Kanani et al. (2010)

magnetopause boundary location tends to exhibit larger variations. The global MHD simulation also suggests that the location of Saturn's magnetopause is less sensitive to changes in the IMF orientation than to the dynamic pressure, a result also obtained from another global simulation study by Fukazawa et al. (2007b).

In addition to the sub-solar locations of the magnetopause and bow shock, another important aspect concerning the shape of the magnetosphere is its dimensions in the dawn-dusk direction and in the north-south direction. Because of the presence of a disk-like current sheet at Jupiter, Jupiter's magnetospheric boundaries are expected to exhibit strong polar flattening (e.g., Slavin et al. 1985; Huddleston et al. 1998; Pilkington et al. 2014), meaning that the magnetosphere extends further from the planet in the dawn-dusk direction than in the north-south direction. Walker et al. (2005) have specifically discussed the issue of polar flattening by use of their Jupiter simulations considering a variety of external conditions. They find that, while polar flattening is present in their simulations, the level of flattening appears to depend on various factors including both the strength and orientation of the IMF and the upstream solar wind dynamic pressure. Their model results suggest that polar flattening is more prominent for lower solar wind dynamic pressure. While more observational data along with modeling efforts are clearly needed to better characterize the polar flattening effect at Jupiter, comparatively little is known about this effect at Saturn. Future studies combining analysis of Cassini measurements with global magnetosphere models certainly need to be undertaken to assess the effect of polar flattening at Saturn. 


\subsubsection{Global Dynamics (Magnetospheric Responses to Solar Wind Driving)}

Global MHD models have also been used extensively to study the dynamics of the giant planet magnetospheres. For rapidly rotating magnetospheres like those of Jupiter and Saturn, the interplay between rotationally driven processes and solar wind driven processes is an important aspect in considering global magnetospheric dynamics. Towards this end, global MHD models have been used to investigate the relative importance of various internal and external processes in driving global magnetospheric dynamics, though the detailed internal magnetodisc force balance involving both thermal and superthermal plasma populations described by Achilleos et al. (2010a,b) cannot be self-consistently modeled in MHD.

For Jupiter, while it is generally thought that the magnetosphere is driven largely by internal processes, the extent to which the solar wind affects the global dynamics of the magnetosphere remains uncertain. Fukazawa et al. (2006) performed a set of global MHD simulations to systematically examine the effect of the IMF and solar wind dynamic pressure on Jupiter's magnetospheric structure and dynamics. They find that the dynamic pressure controls the overall size of the magnetosphere and can sometimes influence the properties of Jupiter's plasma sheet through altering the size of the cushion region, an intermediate region of enhanced magnetic field strength between the dayside magnetopause and the dense plasma sheet (see discussion by Went et al. 2011 and Kivelson and Southwood 2005). As the dynamic pressure becomes larger, the cushion region becomes narrower such that changes in the external solar wind can have more direct and stronger effects on the plasma sheet. The external solar wind conditions are also found in their global simulation to be able to impose strong influences on the global plasma convection and the magnetospheric dynamics.

Among other things, a manifestation of the external influence is the control on the way magnetic reconnection occurs in Jupiter's magnetotail. Their model results show that both the IMF and dynamic pressure play an important role in determining the location of the tail neutral line, with the neutral line being closer to the planet for higher dynamic pressure and stronger IMF cases. The external conditions also affect how frequently tail reconnection takes place. Under low solar wind pressure and weak IMF conditions, tail reconnection tends to occur in a periodic manner with periods of the order of tens of hours. The Galileo Energetic Particle Detectors occasionally observed periodic flow bursts (likely associated with reconnection) in the jovian magnetotail with a periodicity of 2 to 3 days (e.g., Krupp et al. 1998; Woch et al. 2002; Kronberg et al. 2005), however, the origin of these periodic flow bursts is not well understood. The New Horizons spacecraft traversed Jupiter's magnetotail and the Solar Wind Around Pluto (SWAP) instrument showed diverse plasma populations and structures. The quasi-periodic fluctuations seen by SWAP at a 3- to 4-day period were thought to be caused by plasmoids moving down the tail (McComas et al. 2007). Given that the repetitive tail reconnection events seen in the global simulations have periods comparable to that associated with the observed flow bursts, Fukazawa et al. (2006) have argued that it is possible that the observed periodic flow bursts were produced by periodic formation of tail X-line driven by the solar wind under certain external conditions.

While it is still debated whether or not the solar wind significantly impacts Jupiter's magnetosphere, it is clear that Saturn's magnetosphere responds strongly to solar wind forcing (Sect. 4.3.2). The effect of the solar wind forcing on Saturn's magnetosphere has also been studied using global MHD simulations including both single-fluid (Fukazawa et al. 2007a, 2007b; Walker et al. 2011; Zieger et al. 2010; Jia et al. 2012b) and multi-fluid MHD models (Kidder et al. 2012). Some of the modeling studies have focused on the large-scale behavior of the magnetosphere under steady solar wind conditions, while others have considered relatively realistic external conditions by using time-varying solar wind input. For instance, 
Kidder et al. (2012) examined the response of Saturn's magnetosphere to IMF rotation and solar wind pressure enhancement while including the affect of a warped magnetodisc generated by mechanical stresses delivered from the solar wind due to the angle between the solar wind flow and Saturn's spin axis (Arridge et al. 2008; Carbary et al. 2010). They found that both types of external forcing can trigger tail reconnection forming plasmoids in their simulation. Motivated by various observational studies (e.g., Clarke et al. 2005; Crary et al. 2005; Cowley et al. 2005b; Bunce et al. 2005b), Jia et al. (2012b) conducted a global simulation study using a solar wind input that contains features, such as compression and rarefaction, typical of the Corotating Interaction Regions (CIRs) formed in the solar wind near Saturn (Jackman et al. 2008) in order to characterize the dynamical response of the magnetosphere to different types of solar wind disturbances. Among other things, the Jia et al. study specifically investigated the role of tail reconnection in driving dynamics in Saturn's magnetosphere and how tail reconnection takes place under different external conditions.

There are two types of magnetic reconnection identified in the Jia et al. global simulation. The first corresponds to the so-called "Vasyliunas-cycle" reconnection, which is an internal process intrinsic to a rotationally driven magnetosphere (Vasyliunas 1983) where the centrifugal acceleration of mass-loaded flux tubes forced by the planetary rotation gives rise to reconnection on closed magnetic field lines. An important product of this process is the formation of plasmoids, which provide a means for removing plasma from the magnetosphere. The second type identified in the simulation refers to the so-called "Dungey-cycle" reconnection that involves open field lines previously stored in the tail lobes. The two types of reconnection appear to have different field and plasma characteristics in the reconnection products. In particular, the case involving Dungey-cycle reconnection, where reconnection proceeds to the lobe field lines above the plasma sheet, typically results in hotter and more depleted flux tubes with faster bulk flows in the outflows from the reconnection site compared to those produced directly by the Vasyliunas-cycle reconnection. On their return from the tail reconnection site to the dayside, the hot, tenuous, and rapidly moving flux tubes associated with lobe field reconnection may generate significant disturbances in the magnetosphere and the polar ionosphere, particularly on the dawn side, such as generating strong field-aligned currents that would be expected to cause auroral brightening (Badman and Cowley 2007; Mitchell 2015).

The interplay between the Vasyliunas cycle and the Dungey cycle leads to a complex picture of global magnetospheric convection at Saturn. Figure 22 shows an equatorial view of the plasma convection pattern seen in the global simulation of Jia et al. (2012b) under conditions of strong solar wind driving. Although this figure shows only a snapshot from the simulation that should not be viewed as representing a steady state (especially in the case of varying solar wind conditions), it does reveal notable features typically seen when both the Vasyliunas and the Dungey cycles are at work. The pure Vasyliunas-cycle X-line (see the closed magnetic loops shown by the orange lines) is confined to a limited region in the premidnight sector while the Dungey-cycle X-line (across which tailward and planetward fast flows are present), albeit variable both in space and time, is seen mainly in the post-midnight region, adjacent to the Vasyliunas-cycle $\mathrm{X}$-line. In addition to the $\mathrm{X}$-line geometries, another important feature of the resulting convection pattern is that the magnetotail on both the dawnside and duskside flanks contains regions of mass-loaded flux tubes streaming down the tail. These regions appear to be rather important for the loss of magnetospheric plasma, which will be further discussed below. 


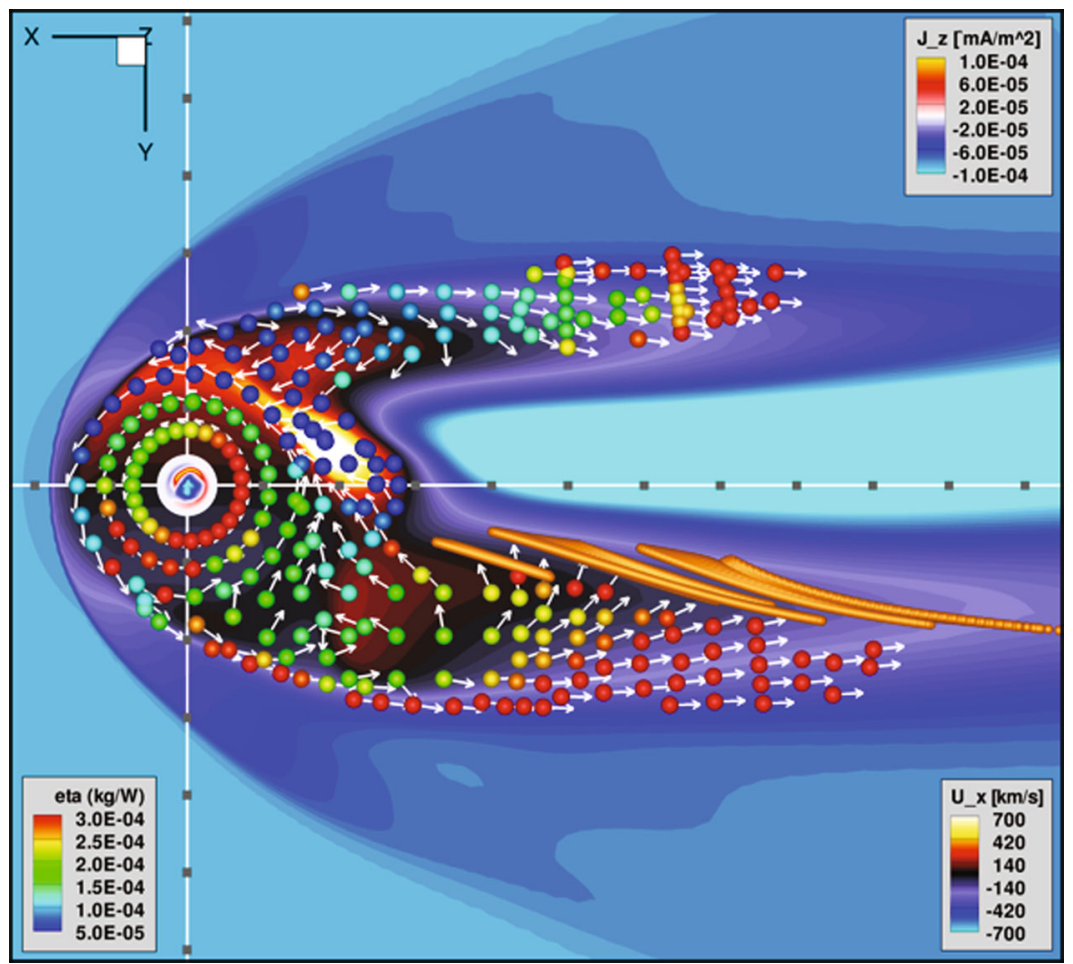

Fig. 22 An equatorial view of the global convection pattern and distribution of plasma content in Saturn's magnetosphere under strong solar driving conditions (from Jia et al. 2012b). The background colors represent contours of the horizontal flow velocity $\left(V_{x}\right)$ and the color contours on a circular disk surrounding Saturn show field-aligned currents intensity in the northern ionosphere (mapped to radial distance of $4 R_{S}$ for illustration purposes). The intersections of sampled closed field lines with the equatorial plane are plotted as balls color-coded with their corresponding flux tube content. Also plotted are unit flow vectors of the closed field lines depicting the direction of their motion. The orange traces show some representative field lines that form closed loops. Grey squares mark off every $10 R_{S}$ along the axes

\subsubsection{Plasma Loss from the Magnetosphere}

For both Jupiter and Saturn, where their moons are continuously adding a significant amount of plasma into the magnetosphere, a fundamental problem is how the magnetospheric plasma is lost from the system. Quantifying such a process through analysis of in-situ measurements turns out to be rather difficult given the highly limited data coverage. Simple order-of-magnitude estimates based on the characteristics of observed tail reconnection and plasmoid events (Bagenal 2007; Bagenal and Delamere 2011) imply that at both planets, plasma loss through large-scale plasmoid release appears insufficient to account for the total loss of plasma required to balance the mass input by internal plasma sources. Global magnetosphere simulations may provide useful insight into how the plasma loss process(es) occur in a rapidly rotating magnetosphere. Kidder et al. (2012) showed that plasmoid formation can be triggered by external forcing (e.g. flipping the IMF direction or a pulse in dynamic pressure). Zieger et al. (2010) and Jia et al. (2012b) have attempted to quantify the mass release processes identified in their global MHD model of Saturn's magnetosphere. Their calculation indicates that on average large-scale plasmoid releases only account for a small 
fraction $(\sim 10 \%)$ of the total loss of plasma added to the magnetosphere by the moon Enceladus and its extended neutral cloud. However, they find that a significant fraction of the planetary plasma appears to be lost through processes (e.g., small-scale plasmoids) near the flanks of the magnetotail that contain flux tubes filled with magnetospheric plasma streaming down the tail (see Fig. 22). The breaking-off of those flux tubes (likely through smallscale plasmoid release) at large distances suggest these regions are crucially important for releasing plasma from the magnetosphere, a situation similar to that proposed by Kivelson and Southwood (2005) in a jovian context. Selective escape of hot plasma due to its kinetic properties was discussed by Sergis et al. (2013). Islands of heavy water groups ions were found in the magnetosheath and upstream on the bow shock, indicating that some plasma can escape at the dayside magnetopause. In addition, Krupp et al. (2002) discusses leakage of energetic particles on Jupiter's dusk flank and Thomsen et al. (2007) assesses superthermal ions in Saturn's foreshock region.

\section{Summary}

The interaction of giant planet magnetodiscs with the solar wind is tied to the momentum transfer rate from the solar wind to the magnetodisc. In steady state, the scale of the interaction must be sufficient to remove plasma supplied by Io $(\sim 100 \mathrm{~s} \mathrm{~kg} / \mathrm{s})$ and by Enceladus $(\sim 10 \mathrm{~s} \mathrm{~kg} / \mathrm{s})$ for Jupiter and Saturn respectively (Delamere and Bagenal 2013). Yet, the details of how plasma is transported from the inner to the outer magnetosphere is poorly understood and remains a fundamental and unsolved problem in magnetodisc physics. Ultimately, magnetic reconnection on both large-scale (Dungey/Vasyliunas cycle) and smallscale (along the flanks of the magnetosphere) together with diffusive processes at the magnetopause boundary facilitate the loss of plasma from the magnetodisc to the solar wind. Auroral signatures provide key diagnostics of this interaction and auroral evidence suggests that large-scale plasmoid formation in the tail is not the primary loss mechanism. Instead, small-scale drizzle along the dusk flank akin to a planetary wind may be the primary pathway (Bagenal 2007; Thomsen et al. 2014). Global-scale MHD simulations produce drizzle along the dusk flank, but we note that this process is a numerical artifact on the grid scale and cannot provide insight into scale-scale physics of the problem. We summarize with the following questions for future research efforts:

- What are the properties of radial plasma transport and magnetic flux circulation in the giant planet magnetodiscs?

- How are thin current sheets generated in the magnetodiscs, allowing reconnection to operate (Vasyliunas 1983; Zimbardo 1991)? What is the relative importance of centrifugal stresses (internal) vs. solar wind-induced stresses (external)?

- How is plasma heated during radial transport?

- What is the prevailing mechanism for mass loss on the dusk flank (i.e. diffusive or smallscale reconnection)?

These are some of the issues that will hopefully be addressed during the end-of-mission polar orbit of the Cassini spacecraft and the arrival of the polar-orbiting Juno spacecraft at Jupiter.

Acknowledgements Peter Delamere acknowledges support by NASA grant NNX13AH309G. Xianzhe Jia is supported by the NASA Cassini Data Analysis Program through grant NNX12AK34G and the NASA Outer Planets Research Program through grant NNX12AM74G, and by the NASA Cassini mission under contract 1409449 with JPL. Licia Ray acknowledges supported by NSF Grant 1064635. Chris Arridge is supported by the "Royal Society University Research Fellowship". 


\section{References}

N. Achilleos, P. Guio, C.S. Arridge, A model of force balance in Saturn's magnetodisc. Mon. Not. R. Astron. Soc. 401, 2349-2371 (2010a). doi:10.1111/j.1365-2966.2009.15865.x

N. Achilleos, P. Guio, C.S. Arridge, N. Sergis, R.J. Wilson, M.F. Thomsen, A.J. Coates, Influence of hot plasma pressure on the global structure of Saturn's magnetodisk. Geophys. Res. Lett. 37, L20201 (2010b). doi:10.1029/2010GL045159

N. Achilleos et al., Space Sci. Rev. (2014, this issue)

B.J. Anderson, T.-D. Phan, S.A. Fuselier, Relationships between plasma depletion and subsolar reconnection. J. Geophys. Res. 102, 9531-9542 (1997). doi:10.1029/97JA00173

C.S. Arridge, N. Achilleos, M.K. Dougherty, K.K. Khurana, C.T. Russell, Modeling the size and shape of Saturn's magnetopause with variable dynamic pressure. J. Geophys. Res. (Space Phys.) 111, A11227 (2006). doi:10.1029/2005JA011574

C.S. Arridge, K.K. Khurana, C.T. Russell, D.J. Southwood, N. Achilleos, M.K. Dougherty, A.J. Coates, H.K. Leinweber, Warping of Saturn's magnetospheric and magnetotail current sheets. J. Geophys. Res. (Space Phys.) 113, A08217 (2008). doi:10.1029/2007JA012963

C.S. Arridge, N. André, C.L. Bertucci, P. Garnier, C.M. Jackman, Z. Németh, A.M. Rymer, N. Sergis, K. Szego, A.J. Coates, F.J. Crary, Upstream of Saturn and Titan. Space Sci. Rev. 162, 25-83 (2011). doi:10.1007/s11214-011-9849-X

C. Arridge, N. Achilleos, Y. Bogdanova, E.J. Bunce, S. Cowley, M. Dougherty, A. Fazakerley, G. Jones, J. Jasinski, K. Khuana, S. Krimigis, N. Krupp, L. Lamy, J. Leisner, E. Roussos, C. Russell, P. Zarka, Cassini observations of Saturn's southern polar cusp. J. Geophys. Res. (2014, submitted)

W.I. Axford, C.O. Hines, A unifying theory of high latitude geophysical phenomena and geomagnetic storms. Can. J. Phys. 39, 1433 (1961)

S.V. Badman, E.J. Bunce, J.T. Clarke, S.W.H. Cowley, J.-C. Gérard, D. Grodent, S.E. Milan, Open flux estimates in Saturn's magnetosphere during the January 2004 Cassini-HST campaign, and implications for reconnection rates. J. Geophys. Res. (Space Phys.) 110, A11216 (2005). doi:10.1029/2005JA011240

S.V. Badman, S.W.H. Cowley, J.-C. Gérard, D. Grodent, A statistical analysis of the location and width of Saturn's southern auroras. Ann. Geophys. 24(12), 3533-3545 (2006)

S.V. Badman, S.W.H. Cowley, Significance of Dungey-cycle flows in Jupiter's and Saturn's magnetospheres, and their identification on closed equatorial field lines. Ann. Geophys. 25, 941-951 (2007). doi:10.5194/angeo-25-941-2007

S.V. Badman, D.J. Andrews, S.W.H. Cowley, L. Lamy, G. Provan, C. Tao, S. Kasahara, T. Kimura, M. Fujimoto, H. Melin, T. Stallard, R.H. Brown, K.H. Baines, Rotational modulation and local time dependence of Saturn's infrared $\mathrm{H}_{3}^{+}$auroral intensity. J. Geophys. Res. (Space Phys.) 117, A09228 (2012a). doi:10.1029/2012JA017990

S.V. Badman, N. Achilleos, C.S. Arridge, K.H. Baines, R.H. Brown, E.J. Bunce, A.J. Coates, S.W.H. Cowley, M.K. Dougherty, M. Fujimoto, G. Hospodarsky, S. Kasahara, T. Kimura, H. Melin, D.G. Mitchell, T. Stallard, C. Tao, Cassini observations of ion and electron beams at Saturn and their relationship to infrared auroral arcs. J. Geophys. Res. 117(A1), A01211 (2012b). doi:10.1029/2011JA017222

S.V. Badman, A. Masters, H. Hasegawa, M. Fujimoto, A. Radioti, D. Grodent, N. Sergis, M.K. Dougherty, A.J. Coates, Bursty magnetic reconnection at Saturn's magnetopause. Geophys. Res. Lett. 40, 10271031 (2013)

S.V. Badman, Saturn's auroral morphology and field-aligned currents during a solar wind compression. Icarus (2014, submitted)

S.V. Badman, G. Branduardi-Raymont, M. Galand, S.L.G. Hess, N. Krupp, L. Lamy, H. Melin, C. Tao, Auroral processes at the giant planets: energy deposition, emission mechanisms, morphology and spectra. Space Sci. Rev. (2014a). doi:10.1007/s11214-014-0042-x

S.V. Badman, C.M. Jackman, J.D. Nichols, J.T. Clarke, J.-C. Gérard, Open flux in Saturn's magnetosphere. Icarus 231, 137-145 (2014b). doi:10.1016/j.icarus.2013.12.004

F. Bagenal, The magnetosphere of Jupiter: coupling the equator to the poles. J. Atmos. Sol.-Terr. Phys. 69, 387-402 (2007). doi:10.1016/j.jastp.2006.08.012

F. Bagenal, P.A. Delamere, Flow of mass and energy in the magnetospheres of Jupiter and Saturn. J. Geophys. Res. (Space Phys.) 116, A05209 (2011). doi:10.1029/2010JA016294

G.E. Ballester, J.T. Clarke, J.T. Trauger, W.M. Harris, K.R. Stapelfeldt, D. Crisp, R.W. Evans, E.B. Burgh, C.J. Burrows, S. Casertano, J.S. Gallagher III, R.E. Griffiths, J.J. Hester, J.G. Hoessel, J.A. Holtzman, J.E. Krist, V. Meadows, J.R. Mould, R. Sahai, P.A. Scowen, A.M. Watson, J.A. Westphal, Time-resolved observations of Jupiter's far-ultraviolet aurora. Science 274, 409-412 (1996)

R.L. Baron, T. Owen, J.E.P. Connerney, T. Satoh, J. Harrington, Solar wind control of Jupiter's H ${ }_{3}^{+}$auroras. Icarus 120, 437-442 (1996). doi:10.1006/icar.1996.0063 
C. Barrow, M. Desch, F. Genova, Solar-wind control of Jupiter decametric radio-emission. Astron. Astrophys. 165(1-2), 244-250 (1986)

E.S. Belenkaya, I.I. Alexeev, M.S. Blokhina, E.J. Bunce, S.W.H. Cowley, J.D. Nichols, V.V. Kalegaev, V.G. Petrov, G. Provan, IMF dependence of Saturn's auroras: modelling study of HST and Cassini data from 12-15 February 2008. Ann. Geophys. 28, 1559-1570 (2010). doi:10.5194/angeo-28-1559-2010

E.S. Belenkaya, S.W.H. Cowley, J.D. Nichols, M.S. Blokhina, V.V. Kalegaev, Magnetospheric mapping of the dayside UV auroral oval at Saturn using simultaneous HST images, Cassini IMF data, and a global magnetic field model. Ann. Geophys. 29, 1233-1246 (2011). doi:10.5194/angeo-29-1233-2011

B. Bonfond, When moons create aurora: the satellite footprints on giant planets, in Auroral Phenomenology and Magnetospheric Processes: Earth and Other Planets. Geophysical Monograph Series, vol. 197 (Am. Geophys. Union, Washington, 2012), pp. 133-140

B. Bonfond, M.F. Vogt, J.-C. Gérard, D. Grodent, A. Radioti, V. Coumans, Quasi-periodic polar flares at Jupiter: a signature of pulsed dayside reconnections? Geophys. Res. Lett. 38(2), L02104 (2011). doi:10.1029/2010GL045981

B. Bonfond, D. Grodent, J.-C. Gérard, T. Stallard, J.T. Clarke, M. Yoneda, A. Radioti, J. Gustin, Auroral evidence of Io's control over the magnetosphere of Jupiter. Geophys. Res. Lett. 39, L01105 (2012). doi:10.1029/2011GL050253

S. Bose, A. Bhattacharya, Solar plasma activated decametric radio emission of non-Io origin from Jupiter magnetosphere. Indian J. Radio Space Phys. 32(1), 43-51 (2003)

G. Branduardi-Raymont, R.F. Elsner, M. Galand, D. Grodent, T.E. Cravens, P. Ford, G.R. Gladstone, J.H. Waite, Spectral morphology of the X-ray emission from Jupiter's aurorae. J. Geophys. Res. (Space Phys.) 113, A02202 (2008). doi:10.1029/2007JA012600

E.J. Bunce, S.W.H. Cowley, T.K. Yeoman, Jovian cusp processes: implications for the polar aurora. J. Geophys. Res. 109(A18), A09S13 (2004). doi:10.1029/2003JA010280

E.J. Bunce, S.W.H. Cowley, S.E. Milan, Interplanetary magnetic field control of Saturn's polar cusp aurora. Ann. Geophys. 23, 1405-1431 (2005a). doi:10.5194/angeo-23-1405-2005

E.J. Bunce, S.W.H. Cowley, D.M. Wright, A.J. Coates, M.K. Dougherty, N. Krupp, W.S. Kurth, A.M. Rymer, In situ observations of a solar wind compression-induced hot plasma injection in Saturn's tail. Geophys. Res. Lett. 32, L20S04 (2005b). doi:10.1029/2005GL022888

E.J. Bunce, C.S. Arridge, J.T. Clarke, A.J. Coates, S.W.H. Cowley, M.K. Dougherty, J.-C. Gérard, D. Grodent, K.C. Hansen, J.D. Nichols, D.J. Southwood, D.L. Talboys, Origin of Saturn's aurora: simultaneous observations by Cassini and the Hubble Space Telescope. J. Geophys. Res. 113(A12), A09209 (2008). doi:10.1029/2008JA013257

J.F. Carbary, N. Achilleos, C.S. Arridge, K.K. Khurana, M.K. Dougherty, Global configuration of Saturn's magnetic field derived from observations. Geophys. Res. Lett. 37, L21806 (2010). doi:10.1029/ 2010GL044622

P.A. Cassak, A. Otto, Scaling of the magnetic reconnection rate with symmetric shear flow. Phys. Plasmas 18(7), 074,501 (2011). doi:10.1063/1.3609771

T.A. Cassidy, R.E. Johnson, Collisional spreading of Enceladus's neutral cloud. Icarus 209, 696-703 (2010). doi:10.1016/j.icarus.2010.04.010

G. Caudal, A self-consistent model of Jupiter's magnetodisc including the effects of centrifugal force and pressure. J. Geophys. Res. 91, 4201-4221 (1986). doi:10.1029/JA091iA04p04201

S. Chandrasekhar, Hydrodynamic and Hydromagnetic Stability (1961)

J.T. Clarke, L. Ben Jaffel, J.-C. Gérard, Hubble Space Telescope imaging of Jupiter's UV aurora during the Galileo orbiter mission. J. Geophys. Res. 103, 20,217-20,236 (1998). doi:10.1029/98JE01130

J.T. Clarke, J.-C. Gérard, D. Grodent, S. Wannawichian, J. Gustin, J. Connerney, F.J. Crary, M.K. Dougherty, W.S. Kurth, S.W.H. Cowley, E.J. Bunce, T.W. Hill, J. Kim, Morphological differences between Saturn's ultraviolet aurorae and those of Earth and Jupiter. Nature 433(7027), 717-719 (2005). doi:10.1038/nature03331

J.T. Clarke, J.D. Nichols, J.-C. Gérard, D. Grodent, K.C. Hansen, W.S. Kurth, G.R. Gladstone, J. Duval, S. Wannawichian, E.J. Bunce, S.W.H. Cowley, F.J. Crary, M.K. Dougherty, L. Lamy, D. Mitchell, W.R. Pryor, K. Retherford, T.S. Stallard, B. Zieger, P. Zarka, B. Cecconi, The response of Jupiter's and Saturn's auroral activity to the solar wind. J. Geophys. Res. 114, A05210 (2009). doi:10.1029/2008JA013694

J.F. Cooper, J.A. Simpson, Sources of high-energy protons in Saturn's magnetosphere. J. Geophys. Res. 85, 5793-5802 (1980). doi:10.1029/JA085iA11p05793

M.M. Cowee, D. Winske, S.P. Gary, Hybrid simulations of plasma transport by Kelvin-Helmholtz instability at the magnetopause: density variations and magnetic shear. J. Geophys. Res. (Space Phys.) 115, A06214 (2010). doi:10.1029/2009JA015011

S. Cowley, E. Bunce, R. Prangé, Saturn's polar ionospheric flows and their relation to the main auroral oval. Ann. Geophys. 22, 1379-1394 (2004). doi:10.5194/angeo-22-1379-2004 
S.W.H. Cowley, E.J. Bunce, Origin of the main auroral oval in Jupiter's coupled magnetosphere-ionosphere system. Planet. Space Sci. 49, 1067-1088 (2001). doi:10.1016/S0032-0633(00)00167-7

S.W.H. Cowley, E.J. Bunce, Corotation-driven magnetosphere-ionosphere coupling currents in Saturn's magnetosphere and their relation to the auroras. Ann. Geophys. 21, 1691-1707 (2003). doi:10.5194/angeo-21-1691-2003

S.W.H. Cowley, E.J. Bunce, T.S. Stallard, S. Miller, Jupiter's polar ionospheric flows: theoretical interpretation. Geophys. Res. Lett. 30, 1220 (2003). doi:10.1029/2002GL016030

S.W.H. Cowley, I.I. Alexeev, E.S. Belenkaya, E.J. Bunce, C.E. Cottis, V.V. Kalegaev, J.D. Nichols, R. Prangé, F.J. Wilson, A simple axisymmetric model of magnetosphere-ionosphere coupling currents in Jupiter's polar ionosphere. J. Geophys. Res. 110(A9), A11209 (2005a). doi:10.1029/2005JA011237

S.W.H. Cowley, S.V. Badman, E.J. Bunce, J.T. Clarke, J.-C. Gérard, D. Grodent, C.M. Jackman, S.E. Milan, T.K. Yeoman, Reconnection in a rotation-dominated magnetosphere and its relation to Saturn's auroral dynamics. J. Geophys. Res. 110(A9), A02201 (2005b). doi:10.1029/2004JA010796

S.W.H. Cowley, J.D. Nichols, D.J. Andrews, Modulation of Jupiter's plasma flow, polar currents, and auroral precipitation by solar wind-induced compressions and expansions of the magnetosphere: a simple theoretical model. Ann. Geophys. 25, 1433-1463 (2007)

S.W.H. Cowley, S.V. Badman, S.M. Imber, S.E. Milan, Comment on "Jupiter: a fundamentally different magnetospheric interaction with the solar wind" by D.J. McComas and F. Bagenal. Geophys. Res. Lett. 35, L10101 (2008). doi:10.1029/2007GL032645

F.J. Crary, J.T. Clarke, M.K. Dougherty, P.G. Hanlon, K.C. Hansen, J.T. Steinberg, B.L. Barraclough, A.J. Coates, J.-C. Gérard, D. Grodent, W.S. Kurth, D.G. Mitchell, A.M. Rymer, D.T. Young, Solar wind dynamic pressure and electric field as the main factors controlling Saturn's aurorae. Nature 433, 720722 (2005). doi:10.1038/nature03333

J.C. Cutler, M.K. Dougherty, E. Lucek, A. Masters, Evidence of surface wave on the dusk flank of Saturn's magnetopause possibly caused by the Kelvin-Helmholtz instability. J. Geophys. Res. (Space Phys.) 116, A10220 (2011). doi:10.1029/2011JA016643

R.B. Decker, S.M. Krimigis, E.C. Roelof, M.E. Hill, T.P. Armstrong, G. Gloeckler, D.C. Hamilton, L.J. Lanzerotti, Voyager 1 in the foreshock, termination shock, and heliosheath. Science 309, 2020-2024 (2005). doi:10.1126/science.1117569

P.A. Delamere, F. Bagenal, Modeling variability of plasma conditions in the Io torus. J. Geophys. Res. 108, 5-1 (2003) doi:10.1029/2002JA009706

P.A. Delamere, F. Bagenal, Solar wind interaction with Jupiter's magnetosphere. J. Geophys. Res. 115, A10201 (2010). doi:10.1029/2010JA015347

P.A. Delamere, F. Bagenal, Magnetotail structure of the giant magnetospheres: implications of the viscous interaction with the solar wind. J. Geophys. Res. (Space Phys.) 118, 7045-7053 (2013). doi:10.1002/2013JA019179

P.A. Delamere, R.J. Wilson, A. Masters, Kelvin-Helmholtz instability at Saturn's magnetopause: hybrid simulations. J. Geophys. Res. (Space Phys.) 116, A10222 (2011). doi:10.1029/2011JA016724

P.A. Delamere, R.J. Wilson, S. Eriksson, F. Bagenal, Magnetic signatures of Kelvin-Helmholtz vortices on Saturn's magnetopause: global survey. J. Geophys. Res. (Space Phys.) 118, 393-404 (2013). doi:10.1029/2012JA018197

M.D. Desch, M.L. Kaiser, Voyager measurements of the rotation period of Saturn's magnetic field. Geophys. Res. Lett. 8(3), 253-256 (1981)

M. Desroche, F. Bagenal, P.A. Delamere, N. Erkaev, Conditions at the expanded Jovian magnetopause and implications for the solar wind interaction. J. Geophys. Res. (Space Phys.) 117, A07202 (2012). doi:10.1029/2012JA017621

K. Dialynas, S.M. Krimigis, D.G. Mitchell, D.C. Hamilton, N. Krupp, P.C. Brandt, Energetic ion spectral characteristics in the Saturnian magnetosphere using Cassini/MIMI measurements. J. Geophys. Res. (Space Phys.) 114, A01212 (2009). doi:10.1029/2008JA013761

J.W. Dungey, Electrodynamics of the outer atmosphere, in Proceedings of the Ionosphere Conference (The Physical Society of London, 1955), p. 225

J.W. Dungey, Interplanetary magnetic field and the auroral zones. Phys. Rev. Lett. 6(2), 47-48 (1961). doi:10. 1103/PhysRevLett.6.47

E. Echer, P. Zarka, W.D. Gonzalez, A. Morioka, L. Denis, Solar wind effects on Jupiter non-Io DAM emissions during Ulysses distant encounter (2003-2004). Astron. Astrophys. 519, A84 (2010). doi:10.1051/0004-6361/200913305

R.F. Elsner, N. Lugaz, J.H. Waite, T.E. Cravens, G.R. Gladstone, P. Ford, D. Grodent, A. Bhardwaj, R.J. MacDowall, M.D. Desch, T. Majeed, Simultaneous Chandra X ray, Hubble Space Telescope ultraviolet, and Ulysses radio observations of Jupiter's aurora. J. Geophys. Res. 110(A9), A01207 (2005). doi:10.1029/2004JA010717 
G.J. Fasel, L.C. Lee, R.W. Smith, A mechanism for the multiple brightenings of dayside poleward-moving auroral forms. Geophys. Res. Lett. 20, 2247-2250 (1993). doi:10.1029/93GL02487

B.L. Fleshman, P.A. Delamere, F. Bagenal, T. Cassidy, The roles of charge exchange and dissociation in spreading Saturn's neutral clouds. J. Geophys. Res., Planets 117, E05007 (2012). doi:10.1029/2011JE003996

L.A. Frank, J.D. Craven, J.L. Burch, J.D. Winningham, Polar views of the earth's aurora with Dynamics Explorer. Geophys. Res. Lett. 9, 1001-1004 (1982). doi:10.1029/GL009i009p01001

K. Fukazawa, T. Ogino, R.J. Walker, Dynamics of the Jovian magnetosphere for northward interplanetary magnetic field (IMF). Geophys. Res. Lett. 32, L03202 (2005). doi:10.1029/2004GL021392

K. Fukazawa, T. Ogino, R.J. Walker, Configuration and dynamics of the Jovian magnetosphere. J. Geophys. Res. (Space Phys.) 111, A10207 (2006). doi:10.1029/2006JA011874

K. Fukazawa, T. Ogino, R.J. Walker, Vortex-associated reconnection for northward IMF in the Kronian magnetosphere. Geophys. Res. Lett. 34, L23201 (2007a). doi:10.1029/2007GL031784

K. Fukazawa, S.-i. Ogi, T. Ogino, R.J. Walker, Magnetospheric convection at Saturn as a function of IMF BZ. Geophys. Res. Lett. 34, 1105 (2007b). doi:10.1029/2006GL028373

K. Fukazawa, T. Ogino, R.J. Walker, A simulation study of dynamics in the distant Jovian magnetotail. J. Geophys. Res. (Space Phys.) 115, A09219 (2010). doi:10.1029/2009JA015228

S.A. Fuselier, H.U. Frey, K.J. Trattner, S.B. Mende, J.L. Burch, Cusp aurora dependence on interplanetary

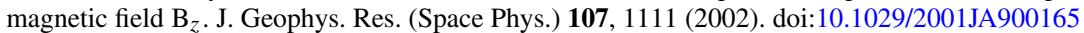

S.A. Fuselier, R. Frahm, W.S. Lewis, A. Masters, J. Mukherjee, S.M. Petrinec, I.J. Sillanpaa, The location of magnetic reconnection at Saturn's magnetopause: a comparison with Earth. J. Geophys. Res. (Space Phys.) 119, 2563-2578 (2014). doi:10.1002/2013JA019684

P.H.M. Galopeau, P. Zarka, D.L. Quéau, Source location of Saturn's kilometric radiation: the KelvinHelmholtz instability hypothesis. J. Geophys. Res. 100, 26,397-26,410 (1995). doi:10.1029/95JE02132

Y.S. Ge, C.T. Russell, K.K. Khurana, Reconnection sites in Jupiter's magnetotail and relation to Jovian auroras. Planet. Space Sci. 58, 1455-1469 (2010). doi:10.1016/j.pss.2010.06.013

F. Genova, P. Zarka, C. Barrow, Voyager and Nancay observations of the Jovian radio-emission at different frequencies - solar-wind effect and source extent. Astron. Astrophys. 182, 159-162 (1987)

J.-C. Gérard, D. Grodent, J. Gustin, A. Saglam, J.T. Clarke, J.T. Trauger, Characteristics of Saturn's FUV aurora observed with the Space Telescope Imaging Spectrograph. J. Geophys. Res. (Space Phys.) 109, A09207 (2004). doi:10.1029/2004JA010513

J.-C. Gérard, E.J. Bunce, D. Grodent, S.W.H. Cowley, J.T. Clarke, S.V. Badman, Signature of Saturn's auroral cusp: simultaneous Hubble Space Telescope FUV observations and upstream solar wind monitoring. J. Geophys. Res. 110(A9), A11201 (2005). doi:10.1029/2005JA011094

G.R. Gladstone, J.H. Waite, D. Grodent, W. Lewis, F.J. Crary, R.F. Elsner, M. Weisskopf, T. Majeed, J. Jahn, A. Bhardwaj, J.T. Clarke, D.T. Young, M.K. Dougherty, S.A. Espinosa, T.E. Cravens, A pulsating auroral X-ray hot spot on Jupiter. Nature 415(6875), 1000-1003 (2002)

D. Grodent, J.T. Clarke, J. Kim, J.H. Waite, S.W.H. Cowley, Jupiter's main auroral oval observed with HSTSTIS. J. Geophys. Res. 108, 1389 (2003a). doi:10.1029/2003JA009921

D. Grodent, J.T. Clarke, J.H. Waite, S.W.H. Cowley, J.-C. Gérard, J. Kim, Jupiter's polar auroral emissions. J. Geophys. Res. 108, 1366 (2003b). doi:10.1029/2003JA010017

D. Grodent, J.-C. Gérard, J.T. Clarke, G.R. Gladstone, J.H. Waite, A possible auroral signature of a magnetotail reconnection process on Jupiter. J. Geophys. Res. (Space Phys.) 109, A05201 (2004). doi:10.1029/2003JA010341

D. Grodent, J.-C. Gérard, S.W.H. Cowley, E.J. Bunce, J.T. Clarke, Variable morphology of Saturn's southern ultraviolet aurora. J. Geophys. Res. (Space Phys.) 110, A07215 (2005). doi:10.1029/2004JA010983

D. Grodent, J.-C. Gérard, A. Radioti, B. Bonfond, A. Saglam, Jupiter's changing auroral location. J. Geophys. Res. 113, 1206 (2008). doi:10.1029/2007JA012601

D. Grodent, A. Radioti, B. Bonfond, J.-C. Gérard, On the origin of Saturn's outer auroral emission. J. Geophys. Res. (Space Phys.) 115, A08219 (2010). doi:10.1029/2009JA014901

D. Grodent, J. Gustin, J.C. Gérard, A. Radioti, B. Bonfond, W.R. Pryor, Small-scale structures in Saturn's ultraviolet aurora. J. Geophys. Res. 116(A9), A09225 (2011). doi:10.1029/2011JA016818

D.A. Gurnett, W.S. Kurth, G.B. Hospodarsky, A.M. Persoon, P. Zarka, A. Lecacheux, S.J. Bolton, M.D. Desch, W.M. Farrell, M.L. Kaiser, H.-P. Ladreiter, H.O. Rucker, P. Galopeau, P. Louarn, D.T. Young, W.R. Pryor, M.K. Dougherty, Control of Jupiter's radio emission and aurorae by the solar wind. Nature 415, 985-987 (2002)

J. Gustin, J.-C. Gérard, D. Grodent, S.W.H. Cowley, J.T. Clarke, A. Grard, Energy-flux relationship in the FUV Jovian aurora deduced from HST-STIS spectral observations. J. Geophys. Res. 109, 10,205 (2004). doi:10.1029/2003JA010365

J. Gustin, S.W.H. Cowley, J.-C. Gérard, G.R. Gladstone, D. Grodent, J.T. Clarke, Characteristics of Jovian morning bright FUV aurora from Hubble Space Telescope/Space Telescope Imaging Spec- 
trograph imaging and spectral observations. J. Geophys. Res. (Space Phys.) 111, A09220 (2006). doi:10.1029/2006JA011730

D.K. Haggerty, M.E. Hill, R.L. McNutt, C. Paranicas, Composition of energetic particles in the Jovian magnetotail. J. Geophys. Res. (Space Phys.) 114, A02208 (2009). doi:10.1029/2008JA013659

K.C. Hansen, A.J. Ridley, G.B. Hospodarsky, N. Achilleos, M.K. Dougherty, T.I. Gombosi, G. Tóth, Global MHD simulations of Saturn's magnetosphere at the time of Cassini approach. Geophys. Res. Lett. 32, L20S06 (2005). doi:10.1029/2005GL022835

H. Hasegawa, M. Fujimoto, T.-D. Phan, H. Rème, A. Balogh, M.W. Dunlop, C. Hashimoto, R. TanDokoro, Transport of solar wind into Earth's magnetosphere through rolled-up Kelvin-Helmholtz vortices. Nature 430, 755-758 (2004). doi:10.1038/nature02799

H. Hasegawa, A. Retinò, A. Vaivads, Y. Khotyaintsev, M. André, T.K.M. Nakamura, W.-L. Teh, B.U.Ö. Sonnerup, S.J. Schwartz, Y. Seki, M. Fujimoto, Y. Saito, H. Rème, P. Canu, Kelvin-Helmholtz waves at the Earth's magnetopause: multiscale development and associated reconnection. J. Geophys. Res. (Space Phys.) 114, A12207 (2009). doi:10.1029/2009JA014042

S.L.G. Hess, E. Echer, P. Zarka, Solar wind pressure effects on Jupiter decametric radio emissions independent of Io. Planet. Space Sci. 70, 114-125 (2012). doi:10.1016/j.pss.2012.05.011

T.W. Hill, Inertial limit on corotation. J. Geophys. Res. 84, 6554-6558 (1979). doi:10.1029/ JA084iA11p06554

T.W. Hill, A.J. Dessler, F.C. Michel, Configuration of the Jovian magnetosphere. Geophys. Res. Lett. 1, 3-6 (1974). doi:10.1029/GL001i001p00003

T.S. Huang, T.W. Hill, Corotation lag of the Jovian atmosphere, ionosphere, and magnetosphere. J. Geophys. Res. 94, 3761-3765 (1989). doi:10.1029/JA094iA04p03761

D.E. Huddleston, C.T. Russell, G. Le, A. Szabo, Magnetopause structure and the role of reconnection at the outer planets. J. Geophys. Res. 102, 24,289-24,004 (1997). doi:10.1029/97JA02416

D.E. Huddleston, C.T. Russell, M.G. Kivelson, K.K. Khurana, L. Bennett, Location and shape of the Jovian magnetopause and bow shock. J. Geophys. Res. 103, 20,075-20,082 (1998). doi:10.1029/98JE00394

C.M. Jackman, N. Achilleos, E.J. Bunce, B. Cecconi, J.T. Clarke, S.W.H. Cowley, W.S. Kurth, P. Zarka, Interplanetary conditions and magnetospheric dynamics during the Cassini orbit insertion fly-through of Saturn's magnetosphere. J. Geophys. Res. (Space Phys.) 110, A10212 (2005). doi:10.1029/2005JA011054

C.M. Jackman, R.J. Forsyth, M.K. Dougherty, The overall configuration of the interplanetary magnetic field upstream of Saturn as revealed by Cassini observations. J. Geophys. Res. (Space Phys.) 113, A08114 (2008). doi:10.1029/2008JA013083

C.M. Jackman, C.S. Arridge, Solar cycle effects on the dynamics of Jupiter's and Saturn's magnetospheres. Solar Phys. 274(1-2), 481-502 (2011). doi:10.1007/s11207-011-9748-z

C.M. Jackman, N. Achilleos, S.W.H. Cowley, E.J. Bunce, A. Radioti, D. Grodent, S.V. Badman, M.K. Dougherty, W. Pryor, Auroral counterpart of magnetic field dipolarizations in Saturn's tail. Planet. Space Sci. 82, 34-42 (2013). doi:10.1016/j.pss.2013.03.010

J. Jasinski, C. Arridge, L. Lamy, J. Leisner, M. Thomsen, D. Mitchell, A. Coates, A. Radioti, G. Jones, E. Roussos, N. Krupp, D. Grodent, M. Dougherty, J. Waite, Cusp observation at Saturn's high latitude magnetosphere by the Cassini spacecraft. Geophys. Res. Lett. (2014). doi:10.1002/2014GL059319

X. Jia, M.G. Kivelson, Driving Saturn's magnetospheric periodicities from the upper atmosphere/ionosphere: magnetotail response to dual sources. J. Geophys. Res. (Space Phys.) 117, A11219 (2012). doi:10.1029/2012JA018183

X. Jia, M.G. Kivelson, T.I. Gombosi, Driving Saturn's magnetospheric periodicities from the upper atmosphere/ionosphere. J. Geophys. Res. (Space Phys.) 117, A04215 (2012a). doi:10.1029/2011JA017367

X. Jia, K.C. Hansen, T.I. Gombosi, M.G. Kivelson, G. Tóth, D.L. DeZeeuw, A.J. Ridley, Magnetospheric configuration and dynamics of Saturn's magnetosphere: a global MHD simulation. J. Geophys. Res. (Space Phys.) 117, A05225 (2012b). doi:10.1029/2012JA017575

S.J. Kanani, C.S. Arridge, G.H. Jones, A.N. Fazakerley, H.J. McAndrews, N. Sergis, S.M. Krimigis, M.K. Dougherty, A.J. Coates, D.T. Young, K.C. Hansen, N. Krupp, A new form of Saturn's magnetopause using a dynamic pressure balance model, based on in situ, multi-instrument Cassini measurements. J. Geophys. Res. (Space Phys.) 115, 6207 (2010). doi:10.1029/2009JA014262

K.K. Khurana, Euler potential models of Jupiter's magnetospheric field. J. Geophys. Res. 102, 11,295-11,306 (1997). doi:10.1029/97JA00563

A. Kidder, C.S. Paty, R.M. Winglee, E.M. Harnett, External triggering of plasmoid development at Saturn. J. Geophys. Res. (Space Phys.) 117, A07206 (2012). doi:10.1029/2012JA017625

M.G. Kivelson, D.J. Southwood, Dynamical consequences of two modes of centrifugal instability in Jupiter's outer magnetosphere. J. Geophys. Res. (Space Phys.) 110, A12209 (2005). doi:10.1029/2005JA011176

S.M. Krimigis, N. Sergis, D.G. Mitchell, D.C. Hamilton, N. Krupp, A dynamic, rotating ring current around Saturn. Nature 450, 1050-1053 (2007). doi:10.1038/nature06425 
E.A. Kronberg, J. Woch, N. Krupp, A. Lagg, K.K. Khurana, K.-H. Glassmeier, Mass release at Jupiter: substorm-like processes in the Jovian magnetotail. J. Geophys. Res. (Space Phys.) 110, A03211 (2005). doi:10.1029/2004JA010777

N. Krupp, J. Woch, A. Lagg, B. Wilken, S. Livi, D.J. Williams, Energetic particle bursts in the predawn Jovian magnetotail. Geophys. Res. Lett. 25, 1249-1252 (1998). doi:10.1029/98GL00863

N. Krupp, J. Woch, A. Lagg, S.A. Espinosa, S. Livi, S.M. Krimigis, D.G. Mitchell, D.J. Williams, A.F. Cheng, B.H. Mauk, R.W. McEntire, T.P. Armstrong, D.C. Hamilton, G. Gloeckler, J. Dandouras, L.J. Lanzerotti, Leakage of energetic particles from Jupiter's dusk magnetosphere: dual spacecraft observations. Geophys. Res. Lett. 29, 1736 (2002). doi:10.1029/2001GL014290

A. Kullen, The connection between transpolar arcs and magnetotail rotation. Geophys. Res. Lett. 27, 73-76 (2000). doi:10.1029/1999GL010675

H.R. Lai, H.Y.W.C.T. Russell, C.S. Arridge, M.K.M.K. Dougherty, Reconnection at the magnetopause of Saturn: perspective from fte occurrence and magnetosphere size. J. Geophys. Res. 117(A5), A05222 (2012)

G.M. Mason, G. Gloeckler, Power law distributions of suprathermal ions in the quiet solar wind. Space Sci. Rev. 172, 241-251 (2012). doi:10.1007/s11214-010-9741-0. http://adsabs.harvard.edu/abs/ 2012SSRv..172..241M

A. Masters, N. Achilleos, C. Bertucci, M.K. Dougherty, S.J. Kanani, C.S. Arridge, H.J. McAndrews, A.J. Coates, Surface waves on Saturn's dawn flank magnetopause driven by the Kelvin-Helmholtz instability. Planet. Space Sci. 57, 1769-1778 (2009). doi:10.1016/j.pss.2009.02.010

A. Masters, N.A. Achilleos, N. Sergis, M.K. Dougherty, M.G. Kivelson, C.S. Arridge, S.M. Krimigis, H.J. McAndrews, M.F. Thomsen, S.J. Kanani, N. Krupp, A.J. Coates, Cassini observations of a Kelvin-Helmholtz vortex in Saturn's outer magnetosphere. J. Geophys. Res. (2010). doi:10.1029/2010JA015351

A. Masters, D.G. Mitchell, A.J. Coates, M.K. Dougherty, Saturn's low-latitude boundary layer: 1. Properties and variability. J. Geophys. Res. (Space Phys.) 116, A06210 (2011). doi:10.1029/2010JA016421

A. Masters, J.P. Eastwood, M. Swisdak, M.F. Thomsen, C.T. Russell, N. Sergis, F.J. Crary, M.K. Dougherty, A.J. Coates, S.M. Krimigis, The importance of plasma $\beta$ conditions for magnetic reconnection at Saturn's magnetopause. Geophys. Res. Lett. 39, L08103 (2012a). doi:10.1029/2012GL051372

A. Masters, N. Achilleos, J.C. Cutler, A.J. Coates, M.K. Dougherty, G.H. Jones, Surface waves on Saturn's magnetopause. Planet. Space Sci. 65, 109-121 (2012b). doi:10.1016/j.pss.2012.02.007

A. Masters, M. Fujimoto, H. Hasegawa, C.T. Russell, A.J. Coates, M.K. Dougherty, Can magnetopause reconnection drive Saturn's magnetosphere? Geophys. Res. Lett. 41, 1862-1868 (2014). doi:10.1002/2014GL059288

B.H. Mauk, J.T. Clarke, D. Grodent, J.H. Waite, C.P. Paranicas, D.J. Williams, Transient aurora on Jupiter from injections of magnetospheric electrons. Nature 415, 1003-1005 (2002)

H.J. McAndrews, C.J. Owen, M.F. Thomsen, B. Lavraud, A.J. Coates, M.K. Dougherty, D.T. Young, Evidence for reconnection at Saturn's magnetopause. J. Geophys. Res. (Space Phys.) 113, A04210 (2008). doi:10.1029/2007JA012581

D.J. McComas, F. Bagenal, Jupiter: a fundamentally different magnetospheric interaction with the solar wind. Geophys. Res. Lett. 34, 20,106 (2007). doi:10.1029/2007GL031078

D.J. McComas, F. Allegrini, F. Bagenal, F. Crary, R.W. Ebert, H. Elliott, A. Stern, P. Valek, Diverse plasma populations and structures in Jupiter's magnetotail. Science 318, 217 (2007). doi:10.1126/science. 1147393

D.J. McComas, F. Bagenal, Reply to comment by S.W.H. Cowley et al. on "Jupiter: a fundamentally different magnetospheric interaction with the solar wind". Geophys. Res. Lett. 35, L10103 (2008). doi:10.1029/2008GL034351

C.J. Meredith, S.W.H. Cowley, K.C. Hansen, J.D. Nichols, T.K. Yeoman, Simultaneous conjugate observations of small-scale structures in Saturn's dayside ultraviolet auroras-implications for physical origins. J. Geophys. Res. (2013). doi:10.1002/jgra.50270

S.E. Milan, B. Hubert, A. Grocott, Formation and motion of a transpolar arc in response to dayside and nightside reconnection. J. Geophys. Res. (Space Phys.) 110, A01212 (2005). doi:10.1029/2004JA010835

R. Mistry, M.K. Dougherty, A. Masters, A.H. Sulaiman, E.J. Allen, Separating drivers of Saturnian magnetopause motion. J. Geophys. Res. (Space Phys.) 119, 1514-1522 (2014). doi:10.1002/2013JA019489

D.G. Mitchell, Injection, interchange and reconnection: energetic particle observations in Saturn's magnetotail, in Magnetotails in the Solar System, ed. by A. Keiling, C. Jackman, P.A. Delamere. AGU Monographs (American Geophysical Union, Washington, 2015). ISBN 978-1-118-84234-8

D.G. Mitchell, S.M. Krimigis, C. Paranicas, P.C. Brandt, J.F. Carbary, E.C. Roelof, W.S. Kurth, D.A. Gurnett, J.T. Clarke, J.D. Nichols, J.-C. Gérard, D.C. Grodent, M.K. Dougherty, W.R. Pryor, Recurrent energization of plasma in the midnight-to-dawn quadrant of Saturn's magnetosphere, and its relationship to auroral UV and radio emissions. Planet. Space Sci. 57, 1732-1742 (2009). doi:10.1016/j.pss.2009.04.002 
T. Moriguchi, A. Nakamizo, T. Tanaka, T. Obara, H. Shimazu, Current systems in the Jovian magnetosphere. J. Geophys. Res. (Space Phys.) 113, A05204 (2008). doi:10.1029/2007JA012751

T.K.M. Nakamura, M. Fujimoto, Magnetic reconnection within rolled-up MHD-scale Kelvin-Helmholtz vortices: two-fluid simulations including finite electron inertial effects. Geophys. Res. Lett. 32, L21102 (2005). doi:10.1029/2005GL023362

T.K.M. Nakamura, M. Fujimoto, A. Otto, Magnetic reconnection induced by weak Kelvin-Helmholtz instability and the formation of the low-latitude boundary layer. Geophys. Res. Lett. 33, L14106 (2006). doi:10.1029/2006GL026318

J. Nichols, S. Cowley, Magnetosphere-ionosphere coupling currents in Jupiter's middle magnetosphere: effect of precipitation-induced enhancement of the ionospheric Pedersen conductivity. Ann. Geophys. 22, 1799-1827 (2004). doi:10.5194/angeo-22-1799-2004

J.D. Nichols, Magnetosphere-ionosphere coupling in Jupiter's middle magnetosphere: computations including a self-consistent current sheet magnetic field model. J. Geophys. Res. (Space Phys.) 116, A10232 (2011). doi:10.1029/2011JA016922

J.D. Nichols, S.W.H. Cowley, D.J. McComas, Magnetopause reconnection rate estimates for Jupiter's magnetosphere based on interplanetary measurements at 5AU. Ann. Geophys. 24, 393-406 (2006)

J.D. Nichols, E.J. Bunce, J.T. Clarke, S.W.H. Cowley, J.-C. Gérard, D. Grodent, W.R. Pryor, Response of Jupiter's UV auroras to interplanetary conditions as observed by the Hubble Space Telescope during the Cassini flyby campaign. J. Geophys. Res. 112(A11), A02203 (2007). doi:10.1029/2006JA012005

J.D. Nichols, J.T. Clarke, J.-C. Gérard, D. Grodent, K.C. Hansen, Variation of different components of Jupiter's auroral emission. J. Geophys. Res. 114, A06210 (2009a). doi:10.1029/2009JA014051

J.D. Nichols, J.T. Clarke, J.C. Gérard, D. Grodent, Observations of Jovian polar auroral filaments. Geophys. Res. Lett. 36, L08101 (2009b). doi:10.1029/2009GL037578

J.D. Nichols, S. Badman, K. Baines, R. Brown, E. Bunce, J. Clarke, S. Cowley, F. Crary, M. Dougherty, J. Grard, A. Grocott, D. Grodent, W. Kurth, H. Melin, D. Mitchell, W. Pryor, T. Stallard, Dynamic auroral storms on Saturn as observed by the Hubble Space Telescope. Geophys. Res. Lett. (2014). doi:10.1002/2014GL060186

K. Nykyri, A. Otto, Plasma transport at the magnetospheric boundary due to reconnection in KelvinHelmholtz vortices. Geophys. Res. Lett. 28, 3565-3568 (2001). doi:10.1029/2001GL013239

K. Nykyri, A. Otto, B. Lavraud, C. Mouikis, L.M. Kistler, A. Balogh, H. Rème, Cluster observations of reconnection due to the Kelvin-Helmholtz instability at the dawnside magnetospheric flank. Ann. Geophys. 24, 2619-2643 (2006)

T. Ogino, R.J. Walker, M.G. Kivelson, A global magnetohydrodynamic simulation of the Jovian magnetosphere. J. Geophys. Res. 103, 225 (1998). doi:10.1029/97JA02247

L. Pallier, R. Prange, More about the structure of the high latitude Jovian aurorae. Planet. Space Sci. 49(1011), 1159-1173 (2001)

C. Paranicas, D.G. Mitchell, S.M. Krimigis, D.C. Hamilton, E. Roussos, N. Krupp, G.H. Jones, R.E. Johnson, J.F. Cooper, T.P. Armstrong, Sources and losses of energetic protons in Saturn's magnetosphere. Icarus 197, 519-525 (2008). doi:10.1016/j.icarus.2008.05.011

G. Paschmann, Recent in-situ observations of magnetic reconnection in near-Earth space. Geophys. Res. Lett. 35, L19109 (2008). doi:10.1029/2008GL035297

T.D. Phan, J.T. Gosling, G. Paschmann, C. Pasma, J.F. Drake, M. Øieroset, D. Larson, R.P. Lin, M.S. Davis, The dependence of magnetic reconnection on plasma $\beta$ and magnetic shear: evidence from solar wind observations. Astrophys. J. Lett. 719, L199-L203 (2010). doi:10.1088/2041-8205/719/2/L199

T.D. Phan, G. Paschmann, J.T. Gosling, M. Oieroset, M. Fujimoto, J.F. Drake, V. Angelopoulos, The dependence of magnetic reconnection on plasma $\beta$ and magnetic shear: evidence from magnetopause observations. Geophys. Res. Lett. 40, 11-16 (2013). doi:10.1029/2012GL054528

V. Pierrard, M. Lazar, Kappa distributions: theory and applications in space plasmas. Sol. Phys. 267, 153-174 (2010). doi:10.1007/s11207-010-9640-2

N.M. Pilkington, N. Achilleos, C.S. Arridge, A. Masters, N. Sergis, A.J. Coates, M.K. Dougherty, Polar confinement of Saturn's magnetosphere revealed by in situ Cassini observations. J. Geophys. Res. (Space Phys.) 119, 2858-2875 (2014). doi:10.1002/2014JA019774

D.H. Pontius, Radial mass transport and rotational dynamics. J. Geophys. Res. 102, 7137-7150 (1997). doi:10.1029/97JA00289

W.R. Pryor, A.I.F. Stewart, L.W. Esposito, W.E. McClintock, J.E. Colwell, A.J. Jouchoux, A.J. Steffl, D.E. Shemansky, J.M. Ajello, R.A. West, C.J. Hansen, B.T. Tsurutani, W.S. Kurth, G.B. Hospodarsky, D.A. Gurnett, K.C. Hansen, J.H. Waite, F.J. Crary, D.T. Young, N. Krupp, J.T. Clarke, D. Grodent, M.K. Dougherty, Cassini UVIS observations of Jupiter's auroral variability. Icarus 178, 312-326 (2005). doi:10.1016/j.icarus.2005.05.021

W.R. Pryor, A.M. Rymer, D.G. Mitchell, T.W. Hill, D.T. Young, J. Saur, G.H. Jones, S. Jacobsen, S.W.H. Cowley, B.H. Mauk, A.J. Coates, J. Gustin, D. Grodent, J.-C. Gérard, L. Lamy, J.D. Nichols, S.M. Krim- 
igis, L.W. Esposito, M.K. Dougherty, A.J. Jouchoux, A.I.F. Stewart, W.E. McClintock, G.M. Holsclaw, J.M. Ajello, J.E. Colwell, A.R. Hendrix, F.J. Crary, J.T. Clarke, X. Zhou, The auroral footprint of Enceladus on Saturn. Nature 472, 331-333 (2011). doi:10.1038/nature09928

A. Radioti, J.-C. Gérard, D. Grodent, B. Bonfond, N. Krupp, J. Woch, Discontinuity in Jupiter's main auroral oval. J. Geophys. Res. 113, 1215 (2008a). doi:10.1029/2007JA012610

A. Radioti, D. Grodent, J.C. Gérard, B. Bonfond, J.T. Clarke, Auroral polar dawn spots: Signatures of internally driven reconnection processes at Jupiter's magnetotail. Geophys. Res. Lett. 35(3) (2008b)

A. Radioti, A.T. Tomás, D. Grondent, J.C. Gérard, J. Gustin, B. Bonfond, N. Krupp, J. Woch, J.D. Menietti, Equatorward diffuse auroral emissions at Jupiter: simultaneous HST and Galileo observations. Geophys. Res. Lett. 36, 7101 (2009a)

A. Radioti, J.-C. Gérard, E. Roussos, C.P. Paranicas, B. Bonfond, D.G. Mitchell, N. Krupp, S.M. Krimigis, J.T. Clarke, Transient auroral features at Saturn: signatures of energetic particle injections in the magnetosphere. J. Geophys. Res. 114, A03210 (2009b). doi:10.1029/2008JA013632

A. Radioti, D. Grodent, J.-C. Gérard, B. Bonfond, Auroral signatures of flow bursts released during magnetotail reconnection at Jupiter. J. Geophys. Res. (Space Phys.) 115, A07214 (2010). doi:10.1029/2009JA014844

A. Radioti, D. Grodent, J.-C. Gérard, S.E. Milan, B. Bonfond, J. Gustin, W. Pryor, Bifurcations of the main auroral ring at Saturn: ionospheric signatures of consecutive reconnection events at the magnetopause. J. Geophys. Res. (Space Phys.) 116, A11209 (2011a). doi:10.1029/2011JA016661

A. Radioti, D. Grodent, J.C. Gerard, M.F. Vogt, M. Lystrup, B. Bonfond, Nightside reconnection at Jupiter: auroral and magnetic field observations from 26 July 1998. J. Geophys. Res. 116, A03221 (2011b). doi:10.1029/2010JA016200

A. Radioti, M. Lystrup, B. Bonfond, D. Grodent, J.-C. Gérard, Jupiter's aurora in ultraviolet and infrared: simultaneous observations with the Hubble Space Telescope and the NASA infrared telescope facility. J. Geophys. Res. (Space Phys.) 118, 2286-2295 (2013a). doi:10.1002/jgra.50245

A. Radioti, E. Roussos, D. Grodent, J.-C. Gérard, N. Krupp, D.G. Mitchell, J. Gustin, B. Bonfond, W. Pryor, Signatures of magnetospheric injections in Saturn's aurora. J. Geophys. Res. (Space Phys.) 118, 19221933 (2013b). doi:10.1002/jgra.50161

A. Radioti, D. Grodent, J.-C. Gérard, B. Bonfond, J. Gustin, W. Pryor, J.M. Jasinski, C.S. Arridge, Auroral signatures of multiple magnetopause reconnection at Saturn. Geophys. Res. Lett. 40, 4498-4502 (2013c). doi:10.1002/grl.50889

A. Radioti, D. Grodent, J. Gérard, S. Milan, R. Fear, C. Jackman, B. Bonfond, W. Pryor, Saturn's elusive transpolar arc. Geophys. Res. Lett. (2014a, submitted)

A.D. Radioti, Grodent, X. Jia, J.-C. Gérard, B. Bonfond, W. Pryor, J. Gustin, D. Mitchell, C. Jackman, A remarkable magnetotail reconnection event at Saturn, as observed by UVIS/Cassini. Icarus (2014b, submitted)

L.C. Ray, Y.-J. Su, R.E. Ergun, P.A. Delamere, F. Bagenal, Current-voltage relation of a centrifugally confined plasma. J. Geophys. Res. (Space Phys.) 114, A04214 (2009). doi:10.1029/2008JA013969

L.C. Ray, R.E. Ergun, P.A. Delamere, F. Bagenal, Magnetosphere-ionosphere coupling at Jupiter: effect of field-aligned potentials on angular momentum transport. J. Geophys. Res. (Space Phys.) 115, A09211 (2010). doi:10.1029/2010JA015423

L.C. Ray, M. Galand, L.E. Moore, B.L. Fleshman, Characterizing the limitations to the coupling between Saturn's ionosphere and middle magnetosphere. J. Geophys. Res. (Space Phys.) 117, A07210 (2012). doi:10.1029/2012JA017735

L.C. Ray, M. Galand, P.A. Delamere, B.L. Fleshman, Current-voltage relation for the Saturnian system. J. Geophys. Res. (Space Phys.) 118, 3214-3222 (2013). doi:10.1002/jgra.50330

E. Roussos, N. Krupp, C.P. Paranicas, P. Kollmann, D.G. Mitchell, S.M. Krimigis, T.P. Armstrong, D.R. Went, M.K. Dougherty, G.H. Jones, Long- and short-term variability of Saturn's ionic radiation belts. J. Geophys. Res. (Space Phys.) 116, A02217 (2011). doi:10.1029/2010JA015954

H.O. Rucker, M. Panchenko, K.C. Hansen, U. Taubenschuss, M.Y. Boudjada, W.S. Kurth, M.K. Dougherty, J.T. Steinberg, P. Zarka, P.H.M. Galopeau, D.J. McComas, C.H. Barrow, Saturn kilometric radiation as a monitor for the solar wind? Adv. Space Res. 42, 40-47 (2008). doi:10.1016/j.asr.2008.02.008

C.T. Russell, Reconnection, in Physics of Solar Planetary Environments (Am. Geophys. Union, Washington, 1975), pp. 526-540

C.T. Russell, R.C. Elphic, Initial ISEE magnetometer results-magnetopause observations. Space Sci. Rev. 22, 681-715 (1978). doi:10.1007/BF00212619

C.T. Russell, M.M. Hoppe, W.A. Livesey, Overshoots in planetary bow shocks. Nature 296, $45-48$ (1982). doi: $10.1038 / 296045 \mathrm{a} 0$

B. Sandel, A. Broadfoot, Morphology of Saturn's aurora. Nature 292, 679-682 (1981)

N. Sergis, S.M. Krimigis, E.C. Roelof, C.S. Arridge, A.M. Rymer, D.G. Mitchell, D.C. Hamilton, N. Krupp, M.F. Thomsen, M.K. Dougherty, A.J. Coates, D.T. Young, Particle pressure, inertial force, and ring 
current density profiles in the magnetosphere of Saturn, based on Cassini measurements. Geophys. Res. Lett. 37, L02102 (2010). doi:10.1029/2009GL041920

N. Sergis, C.M. Jackman, A. Masters, S.M. Krimigis, M.F. Thomsen, D.C. Hamilton, D.G. Mitchell, M.K. Dougherty, A.J. Coates, Particle and magnetic field properties of the Saturnian magnetosheath: presence and upstream escape of hot magnetospheric plasma. J. Geophys. Res. (Space Phys.) 118, 1620-1634 (2013). doi:10.1002/jgra.50164

J.A. Slavin, E.J. Smith, J.R. Spreiter, S.S. Stahara, Solar wind flow about the outer planets-gas dynamic modeling of the Jupiter and Saturn bow shocks. J. Geophys. Res. 90, 6275-6286 (1985). doi:10.1029/JA090iA07p06275

C.G.A. Smith, A.D. Aylward, Coupled rotational dynamics of Saturn's thermosphere and magnetosphere: a thermospheric modelling study. Ann. Geophys. 26, 1007-1027 (2008). doi:10.5194/angeo-26-10072008

C.G.A. Smith, A.D. Aylward, Coupled rotational dynamics of Jupiter's thermosphere and magnetosphere. Ann. Geophys. 27, 199-230 (2009). doi:10.5194/angeo-27-199-2009

B.U.O. Sonnerup, B.G. Ledley, Magnetopause rotational forms. J. Geophys. Res. 79, 4309-4314 (1974). doi:10.1029/JA079i028p04309

D.J. Southwood, The hydromagnetic stability of the magnetospheric boundary. Planet. Space Sci. 16, 587605 (1968). doi:10.1016/0032-0633(68)90100-1

D.J. Southwood, M.G. Kivelson, A new perspective concerning the influence of the solar wind on the Jovian magnetosphere. J. Geophys. Res. 106, 6123-6130 (2001). doi:10.1029/2000JA000236

T.S. Stallard, S. Miller, S.W.H. Cowley, E.J. Bunce, Jupiter's polar ionospheric flows: measured intensity and velocity variations poleward of the main auroral oval. Geophys. Res. Lett. 30, 1221 (2003). doi:10.1029/2002GL016031

T. Stallard, S. Miller, H. Melin, M. Lystrup, S.W.H. Cowley, E.J. Bunce, N. Achilleos, M. Dougherty, Jovianlike aurorae on Saturn. Nature 453, 1083-1085 (2008). doi:10.1038/nature07077

Y.-J. Su, R.E. Ergun, F. Bagenal, P.A. Delamere, Io-related Jovian auroral arcs: modeling parallel electric fields. J. Geophys. Res. (Space Phys.) 108, 1094 (2003). doi:10.1029/2002JA009247

M. Swisdak, B.N. Rogers, J.F. Drake, M.A. Shay, Diamagnetic suppression of component magnetic reconnection at the magnetopause. J. Geophys. Res. (Space Phys.) 108, 1218 (2003). doi:10.1029/2002JA009726

M. Swisdak, M. Opher, J.F. Drake, F. Alouani Bibi, The vector direction of the interstellar magnetic field outside the heliosphere. Astrophys. J. 710, 1769-1775 (2010). doi:10.1088/0004-637X/710/2/1769

T. Terasawa, K. Maezawa, S. Machida, Solar-wind effect on Jupiter non-Io-related radio-emission. Nature 273(5658), 131-132 (1978)

N. Thomas, F. Bagenal, T.W. Hill, J.K. Wilson, The Io Neutral Clouds and Plasma Torus (2004), pp. 561-591

M.F. Thomsen, J.P. Dilorenzo, D.J. McComas, D.T. Young, F.J. Crary, D. Delapp, D.B. Reisenfeld, N. Andre, Assessment of the magnetospheric contribution to the suprathermal ions in Saturn's foreshock region. J. Geophys. Res. (Space Phys.) 112, A05220 (2007). doi:10.1029/2006JA012084

M.F. Thomsen, C.M. Jackman, R.L. Tokar, R.J. Wilson, Plasma flows in Saturn's nightside magnetosphere. J. Geophys. Res. (2014). doi:10.1002/2014JA019912

V.M. Vasyliunas, A survey of low-energy electrons in the evening sector of the magnetosphere with OGO 1 and OGO 3. J. Geophys. Res. 73, 2839-2885 (1968)

V.M. Vasyliunas, Theoretical models of magnetic field line merging. I. Rev. Geophys. Space Phys. 13, 303336 (1975). doi:10.1029/RG013i001p00303

V.M. Vasyliunas, Physics of the Jovian magnetosphere, in Plasma Distribution and Flow (1983), pp. 395-453

M.F. Vogt, M.G. Kivelson, K.K. Khurana, S.P. Joy, R.J. Walker, Reconnection and flows in the Jovian magnetotail as inferred from magnetometer observations. J. Geophys. Res. (Space Phys.) 115, A06219 (2010). doi:10.1029/2009JA015098

M.F. Vogt, M.G. Kivelson, K.K. Khurana, R.J. Walker, B. Bonfond, D. Grodent, A. Radioti, Improved mapping of Jupiter's auroral features to magnetospheric sources. J. Geophys. Res. (Space Phys.) 116, A03220 (2011). doi:10.1029/2010JA016148

J.H. Waite, G.R. Gladstone, W.S. Lewis, R. Goldstein, D.J. McComas, P. Riley, R.J. Walker, P. Robertson, S. Desai, J.T. Clarke, D.T. Young, An auroral flare at Jupiter. Nature 410, 787-789 (2001)

R.J. Walker, T. Ogino, A simulation study of currents in the Jovian magnetosphere. Planet. Space Sci. 51, 295-307 (2003). doi:10.1016/S0032-0633(03)00018-7

R.J. Walker, C.T. Russell, Flux transfer events at the Jovian magnetopause. J. Geophys. Res. 90, 7397-7404 (1985). doi:10.1029/JA090iA08p07397

R.J. Walker, S.P. Joy, M.G. Kivelson, K. Khurana, T. Ogino, K. Fukazawa, The locations and shapes of Jupiter's bow shock and magnetopause, in AIP Conf. Proc., vol. 781 (2005)

R.J. Walker, K. Fukazawa, T. Ogino, D. Morozoff, A simulation study of Kelvin-Helmholtz waves at Saturn's magnetopause. J. Geophys. Res. (Space Phys.) 116, A03203 (2011). doi:10.1029/2010JA015905 
L. Wang, R.P. Lin, C. Salem, M. Pulupa, D.E. Larson, P.H. Yoon, J.G. Luhmann, Quiet-time interplanetary $\sim 2-20 \mathrm{keV}$ superhalo electrons at solar minimum. Astrophys. J. Lett. 753, L23 (2012). doi:10.1088/2041-8205/753/1/L23

D.R. Went, M.G. Kivelson, N. Achilleos, C.S. Arridge, M.K. Dougherty, Outer magnetospheric structure: Jupiter and Saturn compared. J. Geophys. Res. (Space Phys.) 116, A04224 (2011). doi:10.1029/2010JA016045

R.M. Winglee, A. Kidder, E. Harnett, N. Ifland, C. Paty, D. Snowden, Generation of periodic signatures at Saturn through Titan's interaction with the centrifugal interchange instability. J. Geophys. Res. (Space Phys.) 118, 4253-4269 (2013). doi:10.1002/jgra.50397

R.J. Wilson, P.A. Delamere, F. Bagenal, A. Masters, Kelvin-Helmholtz instability at Saturn's magnetopause: Cassini ion data analysis. J. Geophys. Res. (Space Phys.) 117, A03212 (2012). doi:10.1029/ 2011JA016723

J. Woch, N. Krupp, A. Lagg, Particle bursts in the Jovian magnetosphere: evidence for a near-Jupiter neutral line. Geophys. Res. Lett. 29, 1138 (2002). doi:10.1029/2001GL014080

J.N. Yates, N. Achilleos, P. Guio, Influence of upstream solar wind on thermospheric flows at Jupiter. Planet. Space Sci. 61, 15-31 (2012). doi:10.1016/j.pss.2011.08.007

D.T. Young, J.-J. Berthelier, M. Blanc, J.L. Burch, S. Bolton, A.J. Coates, F.J. Crary, R. Goldstein, M. Grande, T.W. Hill, R.E. Johnson, R.A. Baragiola, V. Kelha, D.J. McComas, K. Mursula, E.C. Sittler, K.R. Svenes, K. Szegö, P. Tanskanen, M.F. Thomsen, S. Bakshi, B.L. Barraclough, Z. Bebesi, D. Delapp, M.W. Dunlop, J.T. Gosling, J.D. Furman, L.K. Gilbert, D. Glenn, C. Holmlund, J.-M. Illiano, G.R. Lewis, D.R. Linder, S. Maurice, H.J. McAndrews, B.T. Narheim, E. Pallier, D. Reisenfeld, A.M. Rymer, H.T. Smith, R.L. Tokar, J. Vilppola, C. Zinsmeyer, Composition and dynamics of plasma in Saturn's magnetosphere. Science 307, 1262-1266 (2005). doi:10.1126/science.1106151

P. Zarka, F. Genova, Low-frequency jovian emission and solar-wind magnetic-sector structure. Nature 306, 767-768 (1983)

B. Zieger, K.C. Hansen, Statistical validation of a solar wind propagation model from 1 to 10 AU. J. Geophys. Res. 113(A12), A08107 (2008). doi:10.1029/2008JA013046

B. Zieger, K.C. Hansen, T.I. Gombosi, D.L. De Zeeuw, Periodic plasma escape from the mass-loaded Kronian magnetosphere. J. Geophys. Res. (Space Phys.) 115, A08208 (2010). doi:10.1029/2009JA014951

G. Zimbardo, Collisionless reconnection in Jupiter's magnetotail. Geophys. Res. Lett. 18, 741-744 (1991). doi:10.1029/91GL00472 